\title{
Review
}

Wei Xiao, Hancong Zhang, Kun Luo*, Chaoli Mao and Jianren Fan

\section{Immersed boundary method for multiphase transport phenomena}

https://doi.org/10.1515/revce-2019-0076

Received November 11, 2019; accepted July 7, 2020;

published online December 14, 2020

Abstract: Multiphase flows with momentum, heat, and mass transfer exist widely in a variety of industrial applications. With the rapid development of numerical algorithms and computer capacity, advanced numerical simulation has become a promising tool in investigating multiphase transport problems. Immersed boundary (IB) method has recently emerged as such a popular interface capturing method for efficient simulations of multiphase flows, and significant achievements have been obtained. In this review, we attempt to give an overview of recent progresses on IB method for multiphase transport phenomena. Firstly, the governing equations, the basic ideas, and different boundary conditions for the IB methods are introduced. This is followed by numerical strategies, from which the IB methods are classified into two types, namely the artificial boundary method and the authentic boundary method. Discussions on the implementation of various boundary conditions at the interphase surface with momentum, heat, and mass transfer for different IB methods are then presented, together with a summary. Then, the state-of-the-art applications of IB methods to multiphase flows, including the isothermal flows, the heat transfer flows, and the mass transfer problems are outlined, with particular emphasis on the latter two topics. Finally, the conclusions and future challenges are identified.

Keywords: fully-resolved simulation; heat and mass transfer; immersed boundary method; multiphase flows; transport phenomena.

\section{Nomenclature}

\section{Latin letters}

$\begin{array}{ll}c & \text { concentration } \\ C_{p} & \text { specific heat } \\ d & \text { diameter } \\ D_{m} & \text { mass diffusion coefficient } \\ E & \text { total energy } \\ F \text { or } f & \text { forcing term } \\ F_{d} & \text { drag correlation } \\ G & \text { constraint function } \\ G r & \text { Grashof number } \\ h & \text { mesh width } \\ h & \text { total enthalpy } \\ J & \text { diffusive flux } \\ K_{g} & \text { total species number } \\ m & \text { molar mass } \\ \bar{n} & \text { normal vector } \\ N u & \text { Nusselt number } \\ p & \text { pressure } \\ P & \text { permeable coefficient } \\ P r & \text { Prandtl number } \\ q & \text { heat flux } \\ R a & \text { Rayleigh number } \\ R e & \text { Reynolds number } \\ S_{h} & \text { energy source term } \\ S_{k} & \text { species source term } \\ S_{m} & \text { mass source term } \\ S_{m o} & \text { momentum source term } \\ t & \text { time } \\ T & \text { temperature } \\ U \text { or } u & \text { velocity } \\ U_{s} & \text { velocity of Stefan flow } \\ V & \text { diffusive velocity } \\ W & \text { molar mass } \\ X & \text { location } \\ \bar{X} & \text { Eulerian coordinate } \\ \bar{X} & \text { Lagrangian coordinate } \\ Y & \text { mass fraction } \\ & \end{array}$

\section{Greek letters}

*Corresponding author: Kun Luo, State Key Laboratory of Clean
Energy Utilization, Zhejiang University, Hangzhou 310027, P.R. China,
E-mail: zjulk@zju.edu.cn. https://orcid.org/0000-0002-2583-3776
Wei Xiao, Hancong Zhang, Chaoli Mao and Jianren Fan, State Key
Laboratory of Clean Energy Utilization, Zhejiang University, Hangzhou
310027, P.R. China

$\begin{array}{ll}\alpha & \text { boundary conditions parameter } \\ \alpha & \text { thermal diffusivity } \\ \beta & \text { boundary conditions parameter }\end{array}$ 


$\begin{array}{ll}\Gamma & \text { immersed interface } \\ \delta & \text { Dirac delta function } \\ \delta_{i j} & \text { Kronecker delta } \\ \varepsilon & \text { porosity } \\ \varepsilon & \text { emissivity } \\ \lambda & \text { thermal conductivity } \\ \mu & \text { dynamic viscosity } \\ \rho & \text { density } \\ \sigma & \text { Stefan-Boltzmann constant } \\ \sigma_{i j} & \text { viscous shear stress } \\ \sigma_{w} & \text { wall-normal stress } \\ \bar{\tau} & \text { tangential vector } \\ \tau_{w} & \text { surface shear } \\ \phi & \text { volume fraction } \\ \phi^{2} & \text { Damköhler number } \\ \varphi & \text { any physical variable } \\ \Phi & \text { temperature gradient } \\ \omega & \text { reaction rate } \\ \varpi & \text { species term }\end{array}$

\section{Subscripts}

$\begin{array}{ll}\text { bulk } & \text { bulk property } \\ f \text { or fluid } & \text { fluid property } \\ \text { gas } & \text { gas property } \\ i b & \text { immersed boundary } \\ i, j & \text { direction } i, j \\ k & \text { species } k \\ p & \text { pressureparticle } \\ s \text { or solid } & \text { solid property } \\ 0 & \text { at initial time or at the reference } \\ 1,2,3 & \mathrm{x}, \mathrm{y}, \mathrm{z} \text { directions } \\ \infty & \text { of infinite far or the free-stream }\end{array}$

\section{Acronyms}

$\begin{array}{ll}\text { ALE } & \text { arbitrary Lagrangian-Eulerian } \\ \text { AMR } & \text { adaptive mesh refinement } \\ \text { BB } & \text { bounce-back } \\ \text { CFB } & \text { circulating fluidized bed } \\ \text { CFD } & \text { computational fluid dynamics } \\ \text { CHT } & \text { conjugate heat transfer } \\ \text { DEM } & \text { Discrete element method } \\ \text { DKT } & \text { drafting, kissing, and tumbling } \\ \text { DNS } & \text { direct numerical simulation } \\ \text { FSI } & \text { fluid-structure interaction } \\ \text { FT } & \text { front-tracking } \\ \text { FWI } & \text { flame-wall interaction } \\ \text { HIT } & \text { homogeneous isotropic turbulence } \\ \text { IB method } & \text { immersed boundary method } \\ \text { IIM } & \text { immersed interface method } \\ \text { LBM } & \text { lattice Boltzmann method } \\ \text { LES } & \text { large Eddy simulation } \\ \text { PID } & \text { proportional-integral-derivative } \\ \text { RANS } & \text { Reynolds-averaged Navier-Stokes } \\ \text { RHS } & \text { right-hand side }\end{array}$

\section{Introduction}

Multiphase flows with heat and mass transfer exist widely in a variety of chemical applications, such as coal combustion, conversion and utilization of biomass, physical and chemical adsorption, chemical-loop combustion, fluidized beds, and heterogeneous catalytic reactions. These processes are usually quite complicated with multiscale and multiphysics couplings. A typical type of multiphase flows with heat and mass transfer in a circulating fluidized bed (CFB) reactor is illustrated in Figure 1. The fuel particles in the furnace travel upward and then burn under the lifting and heating by the injected air. During this process, complex momentum, energy and mass couplings happen in particle-fluid, particle-particle, and particle-wall interactions. Taking the particle-fluid interaction as an example, the momentum, temperature, and mass boundary layers can be formed at the surface of fuel particles from which the interphase transport phenomena occur, including the momentum, heat, and mass transfer processes that are driven by fluid mechanics, interphase temperature difference, and concentration difference, respectively. These transport processes sometimes coexist with phase change and chemical reactions and can significantly alter an industrial system's pressure drop, heat transfer efficiency, and operation stability. Therefore, understanding of these complicated processes is of paramount importance in the design and optimization of industrial reactors to achieve higher efficiency and lower pollution emission (Faghri and Zhang 2006).

With the rapid development of computer performance, computational fluid dynamics (CFD) has become a powerful and convincing tool for both scientific research and industrial applications. Generally speaking, there are two traditional approaches to study multiphase flows with heat and mass transfer, namely the Eulerian-Lagrangian approach and the Eulerian-Eulerian approach (Crowe et al. 1996). These two approaches treat particles as Lagrangian points and Eulerian fields respectively based on the assumption that particles are obviously smaller than the resolved flow scales, and both of them ignore the boundary layers formed around the finite-size particles. As a result, closure models such as the multiphase turbulent-viscosity models (Fox 2014) and the interfacial-exchange models (Höhne and Mehlhoop 2014; Rzehak and Krepper 2013) are required, and relevant numerical errors increase. To better consider the microscale particle-fluid interaction, the particle-resolved simulation method is emerging as a high-fidelity tool in simulating particles or objects embedded in flow fields as volumetric bodies (Balachandar and Eaton 2010). Different from the traditional approaches, the particle-resolved methods could 
resolve all flow and particle scales, including the bodyconformal mesh method (Tezduyar et al. 1992), the fictitious domain method (Glowinski et al. 1999), and the immersed boundary method (IB method) (Peskin 1972).

In the framework of the body-conformal mesh method, the interphase coupling conditions could be directly specified because the mesh nodes could conform to the embedded boundary. This method can achieve high resolution due to the accurate representation of the boundary surface (Zhang et al. 2015). The arbitrary Lagrangian-Eulerian (ALE) method is such a method that can easily handle the moving and deforming boundary problems, in which the computational mesh can be switched between the Eulerian and the Lagrangian frameworks arbitrarily (Hu et al. 1992). However, the generation of body-conformal mesh is usually very timeconsuming and complicated, especially when handling complex geometries. This difficulty becomes even more severe when the geometries are moving or deforming so that mesh movement or remeshing techniques are needed (Johnson and Tezduyar 1999). Another defect of the bodyconformal gird method is that the domain decomposition would be sophisticated and time-consuming when largescale parallelization is required (Haeri and Shrimpton 2012). Great efforts have been made to overcome these issues. Recently, Horne and Mahesh (2019a; 2019b) have successfully developed a body-conformal overset method for direct numerical simulation of turbulent flow laden with $O\left(10^{5}\right)$ fully resolved particles under large-scale parallelization.

Conversely, the fixed mesh method could alleviate the aforementioned problems by applying stationary, even Cartesian grids. These grids generally cannot conform to embedded objects so that the Lagrangian markers representing object surfaces are usually implemented. Both fictitious domain method and IB method are fixed mesh methods. However, the fictitious domain method suffers from inaccuracy in capturing viscous shear stresses on solid boundaries as well as high computational costs (Sotiropoulos and Borazjani 2009). Compared with the fictitious domain method, the IB method owns the advantages of implementation efficiency and lower computational costs, which make it quite popular over the past decades. The IB method was firstly proposed by Peskin (1972) in simulations of viscous blood flow in a human heart. In this original work, the simulations were carried out on a uniform Cartesian grid, and the effect of the embedded boundary on the ambient fluid was taken into account by introducing an additional forcing term to the momentum equation. This forcing term was calculated by using the Hooke's law and spread by Lagrangian markers at the boundary surface to surrounding Eulerian grid nodes. Afterward, the IB method has gained more

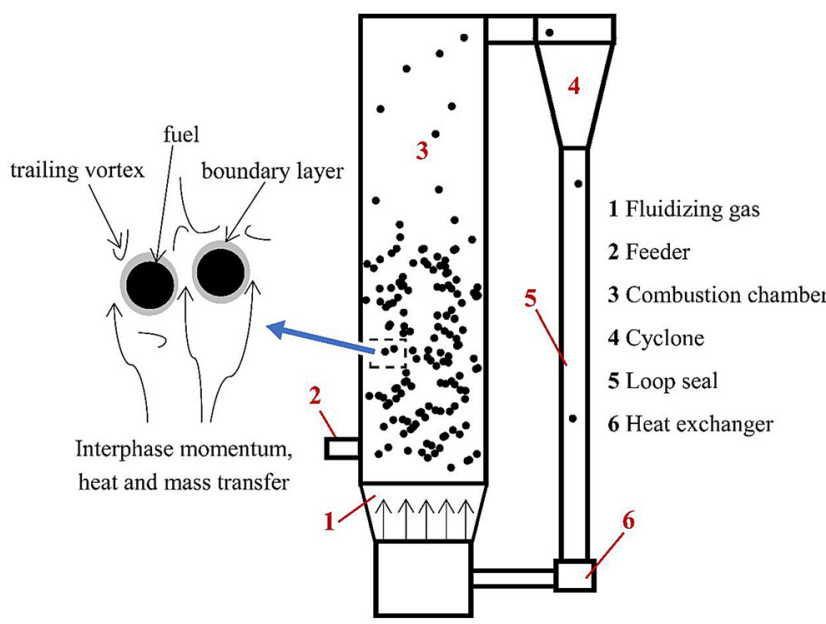

Figure 1: Multiphase transport phenomena in a circulating fluidized bed (CFB).

and more attention and been widely applied in the fields of chemical engineering, materials science, mechanical and energy engineering, and environmental engineering. The application of IB method could range from flow around single stationary object (Kim and Choi 2004) to realistic laboratory-scale fluidized beds loaded with thousands of moving particles (Tang et al. 2016), from laminar isothermal flow past simple geometry (Lai and Peskin 2000) to multiphase turbulent boundary layer flow with heat transfer (Xia et al. 2018), from blood flow in heart valve (Peskin 1972) to mass transfer on coral colonies with complex geometries (Chang et al. 2014). A lot of achievements have been witnessed in the past decades, and some representative progresses are shown in Figure 2. The computational configurations studied with IB methods have become more and more complicated, and the number of loaded fully resolved particles has increased tremendously. Particularly in recent years, fully resolved simulations of heat and mass transfer and chemical reactions with finite-size particles by using the IB method have attracted a lot of attention.

There have been several review articles about the IB method published in the literature. Peskin (2002) provided an in-depth discussion of the mathematical fundamentals of the IB method applicable to simulate the interaction between elastic boundary and viscous incompressible fluid. Iaccarino and Verzicco (2003) reviewed the application of the IB method in simulating incompressible isothermal turbulent flows around complex configurations in the framework of large-eddy simulation (LES) and Reynolds-averaged Navier-Stokes (RANS) approaches. Mittal and Iaccarino (2005) systematically reviewed the formulations of IB methods and classified them into the 


\section{Complexity}

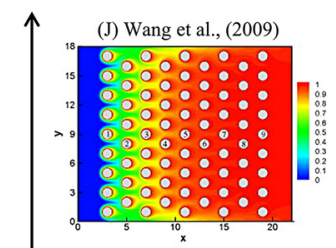

(F) Kang et al., (2009)
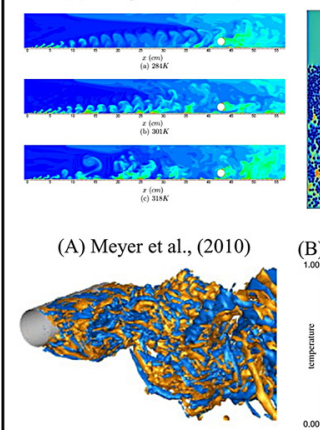

2009
(K) Deen and Kuipers (2014)

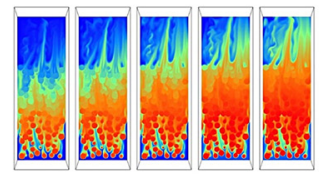

(G) Deen et al., (2012)

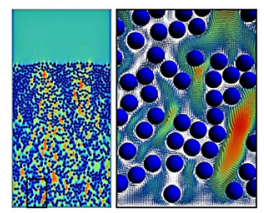

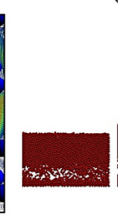

(C)
(L) Luo et al., (2016)

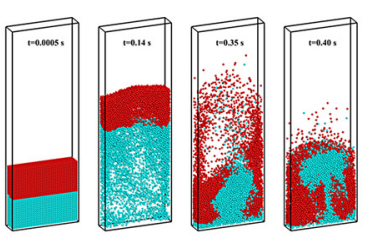

(M) Lu et al., (2019)

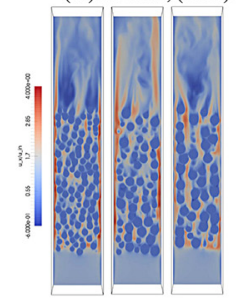

(I) Das et al., (2018)

(H) Tang et al., (2016)
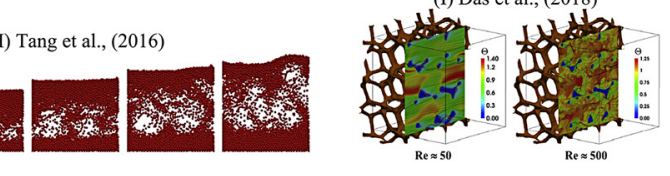

(E) Sulaiman et al., (2019)

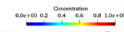
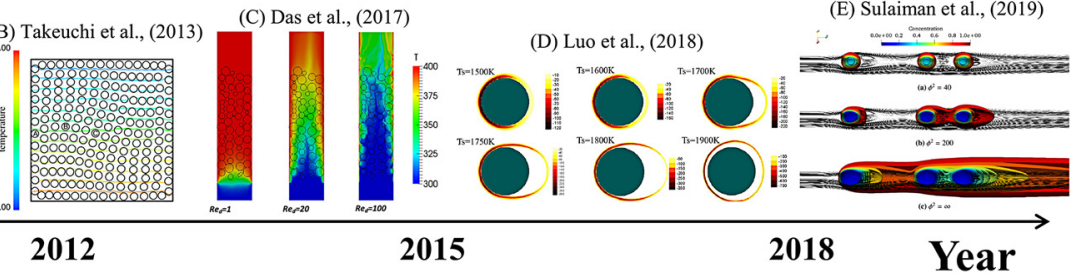

Figure 2: Simulations of multiphase flows at different levels of complexity with IB methods.

(A) Meyer et al. (2010), flow past adiabatic and inert cylinder; (B) Takeuchi et al. (2013), conjugate heat transfer with O(100) particles; (C) Das et al. (2017), conjugate heat transfer with O(100) particles; (D) Luo et al. (2018), single char cylinder combustion; (E) Sulaiman et al. (2019), mass transfer of several tandem spheres; (F) Kang et al. (2009), conjugate heat transfer over a bluff body; (G) Deen et al. (2012), forced convection of flow past O(1000) particles; (H) Tang et al. (2016), O(1000) adiabatic and inert particles in the fluidized bed; (I) Das et al. (2018), flow and conjugate heat transfer through random open-cell solid foams; (J) Wang et al. (2009), forced convection of flow past O(100) cylinders; (K) Deen and Kuipers (2014b), coupled heat and mass transfer with O(1000) particles; (L) Luo et al. (2016a), O(1000) adiabatic and inert particles in fluidized beds; (M) Lu et al. (2019b), coupled heat and mass transfer of flow past O(1000) particles. All figures are reprinted with the permission of Elsevier.

continuous forcing approach and the discrete forcing approach depending on when and how additional forcing terms were calculated. Sotiropoulos and Yang (2014) reviewed the applications of the IB method in fluidstructure interaction (FSI) problems particularly in biofluid mechanics. Maxey (2017) summarized several numerical methods, including a brief introduction to IB methods, in numerical simulations of particulate flows with focuses on the isothermal flows and hydrodynamics. Recently, Huang and Tian (2019) have reviewed the applications of the IB method in FSI problem, with a specific focus on high Reynolds number and supersonic problems. However, all of the aforementioned reviews pay attention to the isothermal problems, and there is currently no review article about the development and application of the IB methods to multiphase flows with heat and mass transfer. Although Deen et al. (2014) reviewed the application of fully resolved simulations on the fluid-particle momentum, heat, and mass transfer, they mainly focused on the dense gas-solid flows, especially for the fluidized bed modeling with simplified boundary conditions. Moreover, the review of numerical methods and applications is mainly based on the studies of their own groups.
Fruitful progresses have recently been achieved by applying IB methods to study multiphase transport phenomena in a wide range of fields. Various heat and mass transfer boundary conditions, which are more realistic (e.g. conjugate heat transfer and finite rate reactions), have also been enforced in fully resolved simulations. All these achievements deserve a comprehensive review.

Therefore, the main objective of this review is to summarize the recent progresses on the IB methods and the relevant applications to studying multiphase transport phenomena with heat and mass transfer. Various kinds of IB methods and relevant improvements are discussed by highlighting the interphase coupling conditions and implementation details for heat, mass transfer, and chemical reactions. The state-of-the-art applications to multiphase transport of momentum, heat, and mass are reviewed. Limitations and future challenges of IB method are also identified. The remainder of the review is organized as follows. In Section 2, the framework of the IB methods including the governing equations, the basic ideas, and the boundary conditions at the interphase surface for multiphase transport phenomena of momentum, energy, and mass is presented. The classification of the IB methods and implementation of 
boundary conditions at the interphase surface, together with a brief summary, are introduced in Section 3. This is followed by Section 4 in which we review the typical application of IB methods in multiphase transport phenomena, including isothermal flows and multiphase flow problems with heat and mass transfer, with particular attention on the latter two topics. Conclusions and the future challenges are given in the last section.

\section{Immersed boundary methods}

\subsection{Governing equations}

Description of a general multiphase flow with heat and mass transfer processes could be very complex. Here we give a set of fully compressible governing equations for two-phase reactive flow, which reads

$$
\begin{gathered}
\frac{\partial \rho}{\partial t}+\frac{\partial\left(\rho u_{j}\right)}{\partial x_{j}}=S_{m}, \\
\frac{\partial\left(\rho u_{i}\right)}{\partial t}+\frac{\partial}{\partial x_{j}}\left(\rho u_{i} u_{j}+p \delta_{i j}-\sigma_{i j}\right)=S_{m o, i}+f_{i b}, \\
\frac{\partial E}{\partial t}+\frac{\partial}{\partial x_{j}}\left((E+p) u_{j}-u_{i} \sigma_{i j}+q_{j}\right)=S_{h}+q_{i b}, \\
\frac{\partial \rho Y_{k}}{\partial t}+\frac{\partial}{\partial x_{j}}\left(\rho u_{j} Y_{k}+\rho V_{k, j} Y_{k}\right)=\omega_{k}+S_{k}+\varpi_{k, i b},
\end{gathered}
$$

where repeated subscripts follow the Einstein notation except for $k$ in Equation (4). $\rho$ is density, $u_{j}$ is the velocity in the $j$-th direction, $x_{j}$ is the Cartesian coordinates in the $j$-th direction, $t$ is time, $p$ is pressure, $\delta_{i j}$ is the Kronecker delta, $\sigma_{i j}$ is the viscous shear stress. $q_{j}$ is the heat flux in the $j$-th direction. $Y_{k}$ and $\omega_{k}$ are mass fraction and reaction rate of the $k$-th species, respectively. $V_{k, j}$ is the diffusion velocity of the $k$-th species in the $j$-th direction. The total energy is $E=\frac{1}{2} \rho u_{i} u_{i}+\rho h-p$, where $h$ is the total enthalpy. $S_{k}$ is the source term of the $k$-th species caused by droplet evaporation, release of volatile matter, or heterogeneous reaction. Because the boundary surface does not always conform to Cartesian mesh, additional forcing, heat, and species terms $f_{i b}, q_{i b}$, and $\varpi_{k, i b}$ are added in the momentum, energy, and species equations to enforce momentum, thermal and species boundary conditions at the embedded body surface. The constructions of $f_{i b}, q_{i b}$, and $\varpi_{k, i b}$ depend on the IB methods. These three terms should account for proper boundary conditions at the interphase surface with enough accuracy and vanish in the flow field far away from the embedded boundary.

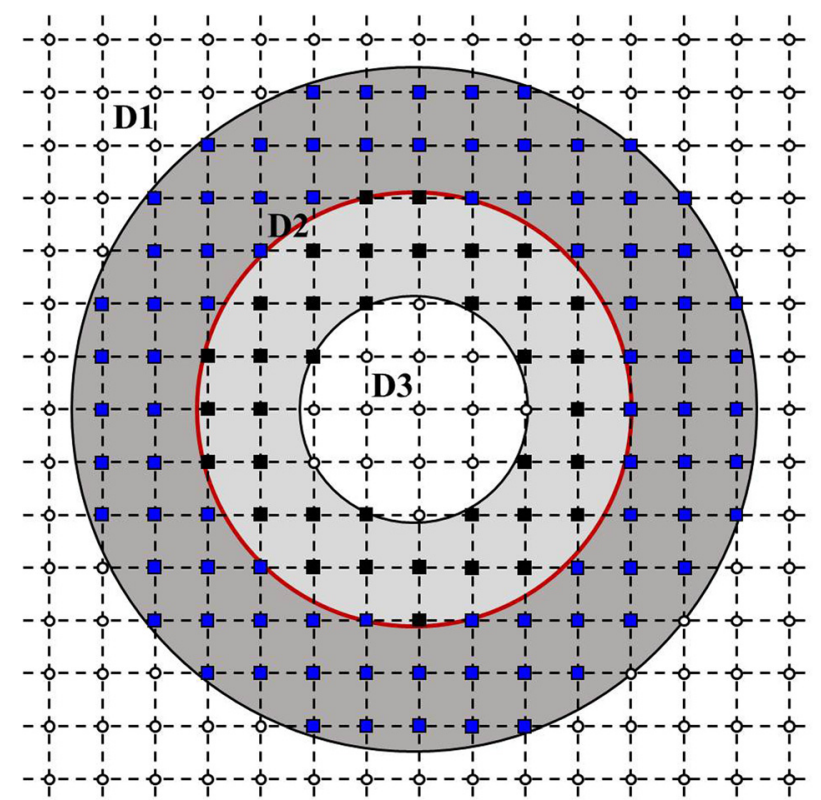

Figure 3: Division of the whole computational domain, the red circle is the true boundary, blue/black squares are exterior/interior grid points in the vicinity of the boundary surface (D2), D1 is the flow region far away from the boundary, and D3 is inside the body far away from the surface.

When the evaporation, release of volatile, or heterogeneous reactions take place at the boundary surface, the Stefan flow would occur, thus giving additional source terms $S_{m}$, $S_{m o, i}$, and $S_{h}$ in the continuity, momentum, and energy equations. It is noteworthy that in these cases, the momentum-energy-species equations are strongly coupled due to local surface conservation of mass and energy. Thus great difficulty arises in the calculation of $S_{m}, S_{m o, i}, S_{h}$, and $S_{k}$.

For a nonreactive incompressible system, the governing equations could be reduced to

$$
\begin{gathered}
\frac{\partial u_{j}}{\partial x_{j}}=0 \\
\rho \frac{\partial u_{i}}{\partial t}+\rho \frac{\partial\left(u_{i} u_{j}\right)}{\partial x_{j}}=-\nabla p+\frac{\partial \sigma_{i j}}{\partial x_{j}}+f_{i b} \\
\rho c_{p}\left(\frac{\partial T}{\partial t}+u_{j} \frac{\partial T}{\partial x_{j}}\right)=\lambda \frac{\partial^{2} T}{\partial x_{j}^{2}}+q_{i b}
\end{gathered}
$$

where $c_{p}$ is the specific heat capacity, $\lambda$ is the thermal conductivity.

\subsection{Basic ideas}

The most distinguishing feature of the IB methods is that it can calculate flows with complex geometry boundary of 
embedded bodies on the Cartesian grids. The interactions between fluid phase and embedded bodies (the effects of interphase coupling hereafter) can be represented by introducing extra terms in the governing equations of momentum, heat, and mass transport equations. With the development of IB methods, the calculation of additional terms is no longer restricted to apply the elastic forcing model as Peskin did (1972), and various methods have been developed to compute these additional terms.

The basic idea of IB methods can be illustrated by Figure 3. Here we use a two-dimensional cylinder as an example. The whole computational domain could be divided into three parts: grid nodes in the flow field that are far away from the boundary, grid nodes near the boundary, which can be either inside (shown in black squares) or outside (shown in blue squares) the boundary, grid nodes inside the embedded body that are away from the boundary. For simplicity, these areas are named as D1, D2, and D3, respectively. In numerical treatments, the effects of the interphase coupling conditions will be imposed in the vicinity of the immersed objects considering the computational efficiency and the negligible influence on the far away flow fields (Peskin 2002). In the original work of Peskin (1972), D1 is the region with larger than two mesh widths away from the boundary, in which the additional forcing terms in the governing equations can be set as zero. Depending on numerical algorithms and forcing models applied in the IB methods, the D1 domain coverage could be varied (Peskin 2002). Unlike D1, the interphase boundary coupling conditions will significantly influence the numerical solution of governing equations in domain D2 (Tseng and Ferziger 2003). Based on different types of boundary conditions at the boundary surface, special treatments are implemented for grid nodes in D2, which will be detailed later. The major difference between various IB methods lies in how the effects of the interphase coupling conditions are incorporated into D2 and how large D2 is.

In this review, we distinguish different kinds of IB methods mainly according to the treatments of D2. If the area of D2 is artificially enlarged compared to the actual object boundary, we call this method the artificial boundary method. The embedded boundary would directly affect the values of surrounding grid nodes in $\mathrm{D} 2$, which results in an artificial boundary representation. If the area of D2 is infinitely small and strictly the same as the actual object boundary, we call this method the authentic boundary method. In this case, the embedded boundary is the same as the actual boundary interface, and the interphase coupling conditions are taken into account by reconstruction of flow properties and local discretization at the immediate boundary interface, which do not directly affect the surrounding grid nodes. As a result, the boundary conditions can be more physically specified, and the conservation properties can be better satisfied.

As for the treatment of grid nodes in D3, there is no mandatory requirement to solve the values at these grid points for most IB methods, because the actual physics inside the boundary is not of interest under most conditions (Haeri and Shrimpton 2012). The governing equations for these grid nodes could be either left unsolved (Nagendra et al. 2014) or solved with proper solvers according to specific problems (Ardekani et al. 2018a).

\subsection{Boundary conditions}

The effects of object boundary on the underlying flow are usually represented by boundary conditions. There are three types of general boundary conditions, namely the constant variable, the constant flux/gradient, and the mix condition, or called as the Dirichlet, Neumann, and Robin-type boundary condition, respectively. These boundary conditions can be generally expressed as a nondimensional linear formulation as

$$
\left.\alpha \varphi\right|_{\Gamma}+\left.\beta \frac{\partial \varphi}{\partial \bar{n}}\right|_{\Gamma}=\left.G\right|_{\Gamma}
$$

where $\varphi$ is the variable, $\bar{n}$ is the normal vector at the immersed interface, $\alpha$ and $\beta$ are parameters related to boundary conditions, $G$ is the known function, which specifies the constraint for $\varphi$ and its gradient at the interface $\Gamma$ (Barozzi et al.2004). By alternating the value of $\alpha$ and $\beta$, it could be easily adapted to express three types of boundary conditions, i.e., setting $\alpha=1, \beta=0$ for the Dirichlet boundary condition or $\alpha=0, \beta=1$ for the Neumann boundary condition or $\alpha \neq 0, \beta \neq 0$ for the Robin boundary condition. All boundary conditions introduced as follows belong to these three types of boundary conditions.

\subsubsection{Momentum transfer boundary conditions}

For momentum transfer at the boundary surface, the no-slip and no-penetration boundary conditions are usually enforced on solid surfaces. Those two boundary conditions indicate there are no relative velocities at the surface (Day 1990), mathematically $\left.u\right|_{\Gamma}=0$ and $\left.v\right|_{\Gamma}=0$, where $\left.u\right|_{\Gamma}$ and $\left.v\right|_{\Gamma}$ are relative velocity in the tangential and perpendicular directions at the interface $\Gamma$.

Another extensively used boundary condition for momentum transfer is the slip boundary condition 
(Kempe et al. 2015). In the cases of rarefied-gas dynamics and non-Newtonian fluids in which the Newtonian law of viscosity cannot hold, modeling the solid surface as slip boundary would be more physically accurate (Lauga et al. 2007). Investigation of flows over such slip surface is quite important as non-Newtonian fluid is frequently encountered in chemical and process engineering (Chhabra and Richardson 1999). Another example is in the numerical study of bubbly flows without any contaminants in a clean system, in which it is usually more appropriate to assume that the surface boundary condition of bubbles is free-slip (Kempe et al. 2015). For slip boundary condition, the tangential slip velocity is determined by the surface shear $\tau_{w}$ and the wall-normal stress $\sigma_{w}$ as $\left.u\right|_{\Gamma}=f\left(\tau_{w}, \sigma_{w}\right)$ (Rao and Rajagopal 1999). The wall-normal velocity $\left.v\right|_{\Gamma}$ is not affected by the slip boundary condition and is still zero for the solid impermeable surface.

\subsubsection{Energy transfer boundary conditions}

For the energy transfer at the interface, additional thermal boundary conditions usually need to be specified. The most extensively used thermal boundary conditions in simulations of multiphase flows are the isothermal and isoflux boundary conditions. The former refers to a Dirichlet boundary condition of $\left.T\right|_{\Gamma}=T_{0}$, and the latter refers to a Neumann boundary condition of $\left.\frac{\partial T}{\partial \bar{n}}\right|_{\Gamma}=\Phi$. When $\Phi=0$, the heat flux across the interface is zero. This is the so-called adiabatic boundary condition.

For more realistic consideration, the heat conduction in the solid side can also be taken into account. In the previous work of Iaccarino et al. (2002), it was shown that heat transfer in heated ribbed passages was significantly modified when the heat conduction through the passage walls was included. Therefore, to include the effect of heat conduction, the thermal boundary conditions should be enforced on both sides of the interface. This kind of heat transfer problem is called as the conjugate heat transfer (CHT) (Perelman 1961). The boundary conditions for CHT are $T_{\text {fluid }}=T_{\text {solid }}$ and $\lambda_{\text {fluid }} \frac{\partial T_{\text {fluid }}}{\partial \bar{n}}=\lambda_{\text {solid }} \frac{\partial T_{\text {solid }}}{\partial \bar{n}}$, which indicate that the continuity of temperature field and balance of heat flux on both sides are associated at the interface. Essentially, the boundary condition for CHT problems is a combination of the Dirichlet type and the Neumann type of boundary conditions. Due to the constraint of temperature and temperature gradient at the boundary, these two boundary conditions must be decoupled first in numerical implementation, which will be discussed in Section 4.

\subsubsection{Mass transfer boundary conditions}

For multiphase flows with mass transfer, the boundary conditions for two typical types of mass transfer problems are discussed. The first type is the mass transfer among a membrane without heterogeneous surface reaction or permeation problem (De Jong et al. 2012a; De Jong et al. 2012b; Miyauchi et al. 2017; Takeuchi et al. 2019; Yao and Mori 2017), and the second type is the mass transfer associated with heterogeneous surface reaction ( $\mathrm{Lu}$ et al. 2019a; Qu and You 2019; Yousefzadeh and Battiato 2019; Zhu et al. 2019).

For the permeation problem, the mass transfer process is governed by the convection and diffusion process across the membrane interface. At the permeable interface, the solute flux in the normal direction is simply related to the concentration difference across the interface as $[c]_{\Gamma}=c_{\Gamma}^{+}-c_{\Gamma}^{-}$(Gong et al. 2014). Thus the solute flux is expressed as $\left.J \cdot \bar{n}\right|_{\Gamma}=-P[c]_{\Gamma}$, where $P$ is the permeable coefficient, which is determined by the properties of the membrane. The diffusive fluxes $J$ across the interface on both sides of the membrane are identical due to mass conservation. For more details of calculating the flux of solute, it is recommended to refer to the Kedem-Katchalsky relations (Kedem and Katchalsky 1958).

For the second mass transfer problem we consider here, the existence of surface heterogeneous reaction would strongly couple the momentum, energy, and mass transfer at the interface. Heterogeneous reaction introduces Stefan flow, which makes the problem more complicated. In fully resolved simulations of interphase mass transfer with the heterogeneous surface reaction, the convection and diffusion of the gas phase are balanced by the reaction rate at the interface as (Kee et al. 2005),

$$
\bar{n} \cdot\left[\rho Y_{k}\left(V_{k}+u_{s}\right)\right]=\omega_{k} W_{k},
$$

where $V_{k}, Y_{k}, \omega_{k}$, and $W_{k}$ are the diffusion velocity, mass fraction, reaction rate, and molar mass of species $k$, and $u_{s}$ is the velocity of the Stefan flow. The velocity of Stefan flow could be obtained by considering the mass balance equation

$$
\bar{n} \cdot u_{s}=\frac{1}{\rho} \sum_{k=1}^{K_{g}} \omega_{k} W_{k} \cdot
$$

The sum of diffusion fluxes of all species at the surface is zero, thus giving

$$
\sum_{k=1}^{K_{g}} Y_{k} V_{k}=0
$$

where $K_{g}$ is the total number of species. At the surface, the heat balance contains the convective and diffuse flux of the 
gas phase, the reaction heat, the radiation, and the heat conduction across the interface as

$$
\begin{aligned}
& \bar{n}\left.\cdot \lambda \nabla T\right|_{\text {gas }}-\sum_{k=1}^{K_{g}} \bar{n} \cdot \rho Y_{k}\left(V_{k}+u_{s}\right) h_{k} \\
&=\sigma \varepsilon\left(T^{4}-T_{0}^{4}\right)+\sum_{k=K s_{0}}^{K_{s}} \omega_{k} W_{k} h_{k}+\left.\bar{n} \cdot \lambda_{\text {bulk }} \nabla T\right|_{\text {bulk }},
\end{aligned}
$$

where $h_{k}$ is the enthalpy of species $k, \sigma$ and $\varepsilon$ are StefanBoltzmann constant and emissivity, respectively. Considering the computational efficiency, some of these parameters or terms in the equations may be simplified or ignored. These will be further discussed in Section 4.

Another noteworthy boundary condition related to the mass transfer problem at the body surface is that of a reactive flow over an embedded body without heterogeneous reactions. Under this condition, there is no surface reaction and mass transfer, thus only no-penetration condition for chemical species should be specified (McGurn et al. 2013), which is $\left.\frac{\partial Y}{\partial \bar{n}}\right|_{\Gamma}=0$ at the boundary surface.

\section{Numerical strategies of IB methods}

With the development of the numerical algorithm and computational capacity, the IB methods have been widely used in studying multiphase flow with heat and mass transfer problems (Deen et al. 2012; Lu et al. 2018a). Compared to Peskin's original IB method (Peskin 1972), the current IB methods have been improved a lot in terms of efficiency, accuracy, and robustness. Different strategies have been developed to handle the complex effects of IB on the underlying flow field. As mentioned before, IB methods can be classified into two types according to the area of D2, which will be introduced here.

\subsection{Artificial boundary method}

As illustrated in Section 2, the core feature of the artificial boundary method is that the numerical manipulations are carried out in an artificially generated region in the vicinity of the immersed object to exert the interphase coupling conditions. The area or layers of modified grid nodes may be different depending on various strategies. According to this definition, the original IB method, the direct-forcing method, and the ghost-cell method belong to this type as these methods share the same idea of modifying the fluid properties at the grid points based on different boundary conditions in an artificial boundary region, which is larger than the actual boundary.

\subsubsection{The original immersed boundary method}

In the pioneering work of Peskin (1972), the embedded body surface is represented by a series of Lagrangian points. At each Lagrangian point, the flow exerts an elastic force $f$ to the body, which is calculated under an elastic forcing model like Hooke's law. This elastic force $f$ is then spread backward to the surrounding Euler grid nodes by a Dirac delta function. Thus the interphase coupling conditions are represented by an additional forcing term $F$ added to the right-hand side of governing equations in this artificially created area near the embedded body. By introducing the additional force $F$, the no-slip boundary condition at the boundary surface could be satisfied. We term this kind of IB method that spreads a Lagrangian force calculated from forcing models to surrounding Euler grids to exert boundary conditions as the original IB method. This method can be expressed in the incompressible Navier-Stokes momentum equation as follows,

$$
\begin{gathered}
\rho\left(\frac{\partial u}{\partial t}+u \cdot \nabla u\right)=-\nabla p+\mu \cdot \nabla^{2} u+F \\
F=\int_{\Gamma} f(s, t) \delta(\bar{x}-\bar{X}(s, t)) \mathrm{d} s
\end{gathered}
$$

where $f(s, t)$ is the force at the $s$-th Lagrangian point $\bar{X}(s, t)$ at the interphase surface, $\delta$ is the Dirac delta function, $\mu$ is the dynamic viscosity, $\Gamma$ is the immersed boundary, $\bar{x}$ is the Eulerian coordinate. The formulation of the Dirac delta function is crucial. It would significantly alter the thickness of the artificial boundary as well as the accuracy of the original IB method. In the original study of Peskin (1972), the projection method was performed to achieve the continuity. The effect of the force term was only considered in the advection-diffusion step, which is shown as the following equation

$$
\rho u *+\rho \delta t\left(u^{n} \cdot \nabla u^{*}-\frac{\mu}{\rho} \nabla^{2} u^{*}\right)=\rho u^{n}+\delta t F
$$

where $u *$ is the intermediate velocity and $u^{n}$ is the velocity at the time step $n$. No additional manipulations were required in the solution of Poisson solver to account for the presence of immersed objects.

One advantage of the original IB method is the consistency in handling the interior and exterior flow fields, i.e., the additional forcing $F$ is added to the grid points on both sides of the boundary. This feature is essential for the case when the flows inside and outside the boundary interface are both of interest. The other advantage is the capability of treating deformable interfaces due to the elastic properties of the boundary specified in the original IB method. These merits make it especially suitable 
for simulations of the FSI problems. Similar ideas are applied to some other methods for simulations of deformable particles too, such as the front-tracking (FT) method (Unverdi and Tryggvason 1992) and the immersed-finite-element method (Saadat et al. 2018; Zhang et al. 2004).

The construction of the Dirac delta function that acts as a kernel in transferring information between Lagrangian and Eulerian fields is quite important and must follow several rules so as to give a smooth and accurate distribution of the additional force. Firstly, the discrete delta function should satisfy the zeroth and first discrete moment conditions so that the conservation of momentum and angular momentum could be achieved in interpolating the Lagrangian force to Eulerian grid nodes. Secondly, the discrete delta function should be continuous and vanish in far fields for computational efficiency. Based on these rules, Peskin (2002) introduced a four-point one-dimensional discrete delta function as $\phi(x / h) / h$, where $h$ is the grid width and $\phi(r)$ reads

$$
\phi(r)= \begin{cases}\frac{1}{8}\left(3-2|r|+\sqrt{1+4|r|-4 r^{2}}\right), & |r| \leq 1 \\ \frac{1}{8}\left(5-2|r|-\sqrt{-7+12|r|-4 r^{2}}\right), & 1 \leq|r| \leq 2 \\ 0, & 2 \leq|r|\end{cases}
$$

Another popular $\phi(r)$ function is formulated as (Peskin 1977),

$$
\phi(r)=\left\{\begin{array}{l}
\frac{1}{4}\left(1+\cos \left(\frac{\pi r}{2}\right)\right), \quad-2 \leq r \leq 2 \\
0, \text { otherwise }
\end{array}\right.
$$

However, the rules for constructing discrete delta function are not unique. In the framework of locally refined and staggered grid system, Roma et al. (1999) modified these rules into weaker forms and proposed a discrete delta function with three-point width. Stokie (1997) considered the second discrete moment condition and proposed a new discrete delta function with better accuracy but more computational cost. Based on these rules, a lot of efforts have been made in comparatively studying the performance of specific delta functions and/or constructing a proper delta function with good performance (Bao et al. 2016; Delouei et al. 2015; Kang and Hassan 2011; Lee and Kim 2012; Yang et al. 2009).

Besides the original model developed by Peskin, many other models have been developed to calculate the surface Lagrangian forcing, such as the PID (proportional-integralderivative) controller forcing model (Goldstein et al. 1993), the penalty IB method (Kim and Peskin 2007; Kim and Peskin 2009), and the volume penalty method (Angot et al. 1999;
Kadoch et al. 2012). According to the classification of Mittal and Iaccarino (2005), the original IB method belongs to the continuous forcing method, where the force terms are firstly calculated according to some constitutive equations and then interpolated into the governing equation of the whole flow field before discretization. As a result, it is independent of the spatial discretization. On the contrary, the force term in the discrete forcing method is introduced only in the boundary region after the discretization of the governing equations, which will be discussed in the following sections.

\subsubsection{The direct-forcing method}

The direct-forcing method was originally derived by MohdYusof (1997), but was first termed by Fadlun et al. (2000). It is also called as the discrete forcing method according to Mittal and Iaccarino (2005). In the direct-forcing method, the presence of the immersed object is represented through introducing additional forcing terms to the governing equations after numerical discretization. This forcing is direct in the sense that it is directly solved from the discrete momentum equations with the desired value of velocity imposed at the IB (Kajishima et al. 2001), rather than from constitutive models as applied in the original IB method. Therefore, the discretization scheme has a great influence on the accuracy, stability, and the conservation performance of the direct-forcing IB method.

The basic principle of the direct-forcing method is described as follows. For temporal advancement of the incompressible Navier-Stokes equation, one has

$$
\frac{u^{n+1}-u^{n}}{\Delta t}=R H S^{n+1 / 2}+F^{n+1 / 2},
$$

where $u^{n}$ is the velocity at the $n$th time step and $u^{n+1}$ for the $(n+1)$-th time step, $F^{n+1 / 2}$ is the boundary force, $R H S^{n+1 / 2}$ contains convective, viscous, and pressure gradient terms at the right-hand side of the momentum equation at the intermediate time step between $n$ and $n+1$ time step. $R H S^{n+1 / 2}$ could be represented by the prediction velocity $u *$ as $u *=u^{n}+R H S^{n+1 / 2} \Delta t$. At the interphase boundary, to yield $u^{n+1}=u_{d}\left(u_{d}\right.$ represents the desired velocity at the boundary), the solution can be used backward to give

$$
F^{n+1 / 2}=\frac{u_{d}-u^{n}}{\Delta t}-R H S^{n+1 / 2}=\frac{u_{d}-u *}{\Delta t} .
$$

Under this formulation, the forcing term $F^{n+1 / 2}$ that directly compensates the error between the calculated velocity and the desired velocity on the IB is derived from the discrete-time Navier-Stokes equations. The fractionalstep method is often used to enforce continuity. When calculating the intermediate velocity in the advection- 
diffusion step, the force term is involved (Uhlmann 2005), which is similar to the treatment in the original IB method. The $k$-th Runge-Kutta step is shown as

$$
\begin{gathered}
\mathrm{u}^{\prime}=\mathrm{u}^{k-1}+\Delta t\left(2 \alpha_{k} \nu \nabla^{2} \mathrm{u}^{k-1}-2 \alpha_{k} \nabla p^{k-1}\right. \\
\left.-\gamma^{k}[(\mathrm{u} \cdot \nabla) \mathrm{u}]^{k-1}-\zeta_{k}[(\mathrm{u} \cdot \nabla) \mathrm{u}]^{k-2}\right), \\
\nabla^{2} \mathrm{u}^{*}-\frac{\mathrm{u}^{*}}{\alpha_{k} v \Delta t}=-\frac{1}{v \alpha_{k}}\left(\frac{\mathrm{u}^{\prime}}{\Delta t}+\mathrm{f}^{k}\right)+\nabla^{2} \mathrm{u}^{k-1}, \\
\nabla^{2} \phi^{k}=\frac{\nabla \cdot \mathrm{u}^{*}}{2 \alpha_{k} \Delta t}, \\
\mathrm{u}^{k}=\mathrm{u}^{*}-2 \alpha_{k} \Delta t \nabla \phi^{k},
\end{gathered}
$$

where $\alpha_{k}, \gamma^{k}$, and $\zeta_{k}$ are coefficients of Runge-Kutta scheme and $\phi$ is the pseudo-pressure (Rai and Moin 1991). The force term $f$ is zero in the original fractional-step method without IB.

The idea of calculating the direct-forcing term is easily understood, while the key issue is how to spread this forcing term to the Eulerian flow field due to the employment of non-body-conformal grids in the IB methods. Many schemes have been proposed to overcome this issue (Chiu et al. 2010; Fadlun et al. 2000; Kajishima et al. 2001; Kim et al. 2001; Luo et al. 2007a; Mohd-Yusof 1997; Silva et al. 2003; Zhang and Zheng 2007). However, it was pointed out by Uhlmann (2005) that the interpolation method applied by Fadlun et al. (2000) could lead to the spurious force oscillations due to insufficient smoothing (this feature is more related to the ghost-cell method and will be further discussed in the next section), which makes the method not suitable for particulate flows with moving boundaries. One important modification to the directforcing method is made by Uhlmann (2005) by combining the direct-forcing method with the original IB method. The basic idea is similar to the original IB method by representing the boundary by a series of Lagrangian points, each of which exerts a direct forcing to the surrounding flow fields both inside and outside the boundary. Similarly, a regularized delta function is used as the kernel for smoothly transferring quantities between Lagrangian and Eulerian grid points. Employment of this regularized delta function could effectively damp the pressure oscillations when simulating moving particles (Uhlmann 2005; Yang et al. 2009).

However, the velocity on the Lagrangian points at the interphase boundary may not adequately satisfy the no-slip boundary condition due to the mutual influence of the direct forcing at the neighboring points through the interpolation of the Dirac delta function in the directforcing method of Uhlmann (2005). After the additional force added, one desires to get $u^{n+1}=u_{d}$ but always gets $u^{n+1} \neq u_{d}$ instead. Although $u^{n+1} \approx u_{d}$ could be achieved after a long period, the no-slip boundary condition may be still unsatisfactory. To remedy this problem, Luo et al. (2007b) and Wang et al. (2008) proposed the multi-directforcing method to reduce the difference between $u^{n+1}$ and $u_{d}$ by iteratively updating the velocity $u^{n+\Delta t_{1}}$ at the sub-time step $n+\Delta t_{1}$ based on the difference between $u^{n+\Delta t_{1}}$ and $u_{d}$. This iterative method can largely improve the numerical accuracy in imposing the no-slip and no-penetration interphase coupling conditions (Luo et al. 2007b). Wang et al. (2008) found out that three additional direct-forcing iterations could reduce the $l_{2}$-norm of the velocity at the surface Lagrangian points by nearly $90 \%$. In the study of Kempe and Fröhlich (2012), they found that for the flow past a single cylinder, three additional forcing loops could reduce nearly $95 \%$ velocity error at the cylinder surface, with only $8 \%$ computational cost increased. Tang et al. (2014) tested the performance of different forcing loop numbers in simulations of flow past an array of spheres and found that two additional iterations could reduce nearly $95 \%$ of the error of the nondimensional drag force.

Both the direct-forcing method of Uhlmann (2005) and the original IB method of Peskin (1972) apply the Dirac delta function to spread the Lagrangian forcing. One significant disadvantage of using the Dirac delta function is that the effective particle diameter is increased, resulting in inauthentic boundary capturing (Breugem 2012). Using the Dirac delta function would also overestimate the drag force experienced by the fluid phase. To alleviate this issue, some studies proposed to retract the Lagrangian points slightly inward to correct the boundary size, and a better drag force and a more accurate flow field could be obtained (Breugem 2012; Luo et al. 2019; Tang et al. 2014).

Besides the direct-forcing method of Uhlmann (2005), some other popular direct-forcing methods are also briefly introduced here. Nakayama and Yamamoto (2005) developed a smoothed-profile method to model the particle phase as Eulerian fields of particle concentration and velocity, which are all zero except in the immediate vicinity of the IB. Based on these Eulerian fields, additional forcing term is added to the governing equation to represent the presence of the IB (Jafari et al. 2011; Mino et al. 2017). Tenneti et al. (2011) developed a particle-resolved uncontaminated fluid reconcilable immersed boundary method (PUReIBM), which successfully alleviates the smear of boundary by restricting the additional direct-forcing term only to the grid points inside the particles, while maintaining the solution of the NavierStokes equation uncontaminated in the fluid phase. 


\subsubsection{The ghost-cell method}

The idea of the ghost-cell IB method originates from the studies of Mohd-Yusof (1997) and Fadlun et al. (2000). They pointed out that the explicit forcing terms in governing equations could be replaced equivalently by reconstructing the velocity field in the vicinity of the boundary region in the time advancement of the governing equations. Later on, Tseng and Ferziger (2003) further used the ghost cells inside the boundary to implicitly represent the direct forcing terms and named the method as the ghost-cell IB method.

The ghost-cell method owns the distinguishing feature of not requiring the explicit addition of forcing terms to the governing equations (Mittal and Iaccarino 2005). The values of the ghost cells are determined by interpolation of boundary conditions and mirror points. The mirror points, also called as image points in some literatures, are fluid points inside the boundary, whose values are interpolated from the surrounding Eulerian fluid grids. By directly solving the governing equations in the vicinity of the IB without any additional terms, the presence of the immersed object could be represented with the help of these reconstructed ghost cells. As for the more interior grid points (than the ghost cells) inside the boundary, their values could be generally left unsolved (Nagendra et al. 2014; Tseng and Ferziger 2003). In the time advancement of the fractional-step method, the detection of boundary interface and determination of values of ghost cells should be carried out before calculating the intermediate velocity. With the help of the ghost cells, the predicted velocity fields including those at the vicinity of the IB can be obtained. Therefore, the pressure Poisson equation could be solved straightforwardly, together with the update of the velocity fields.

The interpolation scheme is an essential component in the implementation of the ghost-cell method. Values at both the mirror points and the ghost cells are determined by interpolation. Many interpolation and extrapolation schemes are available in constructing the ghost-cell method, such as the linear interpolation (Gilmanov et al. 2003; Mittal et al. 2008), the bilinear/trilinear interpolation (Luo et al. 2008; Luo et al. 2016b; Pan 2010; Seo and Mittal 2011a), the high-order interpolation (Seo and Mittal 2011a; Xia et al. 2014), and even the wall function model (Bernardini et al. 2016). Utilizing a suitable interpolation/ extrapolation scheme with higher accuracy and low computational cost is quite crucial in this method.

Pressure oscillation in moving/deformable boundaries is another frequently investigated issue for the ghost-cell methods. Just as commented by Uhlmann (2005), the interpolation procedures of Fadlun et al. (2000) relating values at fixed grid nodes and values at arbitrarily located boundaries can lead to spurious force oscillations due to insufficient smoothing. Some potential sources have been explored, such as implementation of the pressure boundary conditions at the IB (Mark and van Wachem 2008; Shinn et al. 2009), violation of geometric conservation law (Seo and Mittal 2011b), spatial discontinuity in pressure when a grid point inside the solid region becomes that of fluid and temporal discontinuity of velocity vice versa, namely "fresh cell" and "dead cell," respectively (Lee et al. 2011; Seo and Mittal 2011b). Many researchers have paid attention to this problem of the ghost-cell method. Improvements include combining the ghost-cell method with a cut-cell IB method (Merlin et al. 2013; Seo and Mittal 2011b), introducing additional mass source/sink terms together with a larger time step in fully implicit numerical scheme (Lee and You 2013), correcting the fictitious velocity and pressure fields at the ghost cells to ensure no penetration and mass conservation through boundaries (Mark et al. 2013).

\subsection{Authentic boundary method}

Compared with the artificial boundary method, the authentic boundary method considers only values at the actual boundary surface. As a result, better representation of boundary surface and conservation properties could be achieved without the inclusion of a finite area to impose the interphase coupling conditions. Two typical authentic boundary methods are the cut cell method and the immersed interface method (IIM).

\subsubsection{The cut-cell method}

The cut-cell method is a successful application of the IB methods in the finite-volume framework. It was first developed by Udaykumar et al. (1997) and Ye et al. (1999). In this method, the cells near the boundary are cut and reshaped to fit the boundary curves, and the boundary conditions are imposed on the reshaped boundary interface. For the grid points inside the geometry, they could be just left unsolved if the interior flows are not of interest or solved based on specific problem of interest due to the convenience of the finite-volume solver (Ye et al. 1999). One of the most highlighted advantages of the cut-cell method is the strict conservation of mass and momentum in the vicinity of the embedded boundary due to the usage of the finite-volume solver (Schneiders et al. 2013). This 
makes the cut-cell method a good choice in maintaining a divergence-free velocity field (Muldoon and Acharya 2008) and damping the spurious unphysical pressure oscillation (Cecere and Giacomazzi 2014; Seo and Mittal 2011b) in incompressible flows.

However, in the generation of cut cells, exceptional cells of small size may exist, which could result in impractical small time step and numerical instability (Yang et al. 1999). Different techniques have been developed to handle this problem. Zeuuw (1993) used a special local time-stepping scheme to overcome the small time step problem. Ye et al. (1999) reshaped and merged cut cells to form a bigger cell. Muralidharan and Menon (2016) developed a novel cell clustering algorithm for polynomial reconstruction in the vicinity of small cells to achieve smoothness. Kirkpatrick and Armfield (2003) introduced a cell linking method to tag cells as master and slave cells to enable the same treatment for all cells.

When dealing with the time advancement, the boundary cells need to be reshaped into the trapezoidal boundary cells according to the intersection of the boundary with the Cartesian mesh, as shown in Figure 4. In most cases, the face centers of trapezoidal boundary cells are not located at the midpoint of neighboring cell centers. Therefore, to maintain the accuracy, some complicated polynomial interpolating functions may be employed instead of using linear approximation. Consequently, the convergence of the Poisson equation becomes more difficult as the stencil needed by the interpolating functions of the trapezoidal boundary cells cannot be included in the line-successiveoverrelaxation (SOR) sweeps.

In addition, there exist some inherent difficulties in employing the cut-cell method. The cut-cell generation is very challenging when dealing with complex threedimensional problems since complicated cut-cell geometry may occur. To capture the boundary correctly with high efficiency, the cut-cell method is usually combined with the level-set method to identify the boundary interface (Cheny and Botella 2010; Schneiders et al. 2013). Another problem is that numerical oscillations will occur if the cut-cell method for fixed bodies is directly applied to solve moving boundary problems (Schneiders et al. 2013). To overcome this problem, Chung (2006) developed a fully implicit scheme for convective and diffusion terms integration along with a pressure-free projection method to handle moving boundary problems. Muralidharan and Menon (2018) combined the cell clustering technique with the cell mass redistribution for moving boundaries to ensure mass and momentum conservation near the boundary.

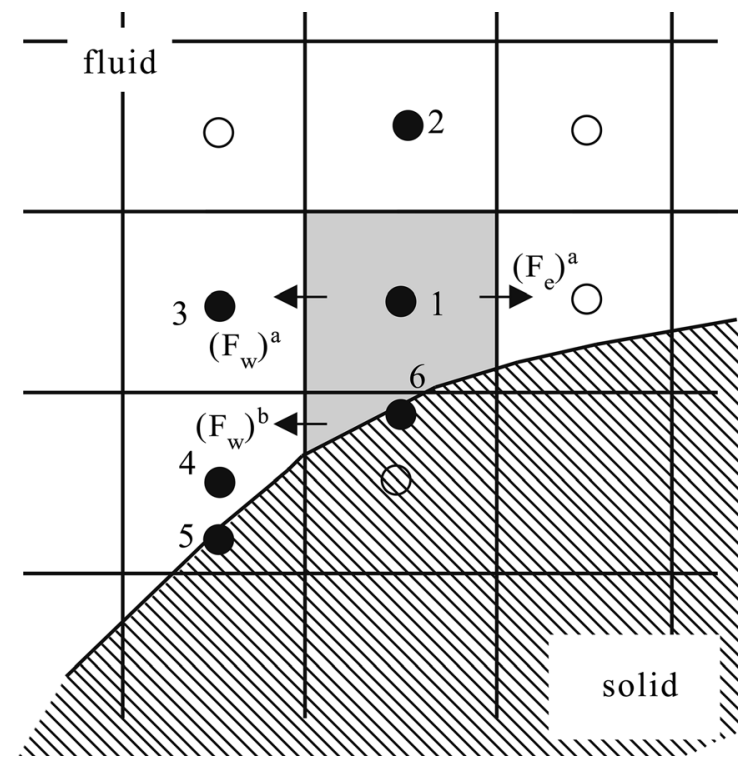

Figure 4: The trapezoidal boundary cells and stencil points for interpolation to obtain the flux. Reprinted from Udaykumar (2001) with the permission of Elsevier.

\subsubsection{The immersed interface method}

In the original IB method of Peskin (1972) and the directforcing IB method of Uhlmann (2005), the Dirac delta function would cause diffusive boundary problems, as discussed before. To alleviate this problem, Leveque and Li (1994) first proposed the IIM for elliptical equations to incorporate the forcing terms into jump conditions at the boundary interface and modify the numerical discretization algorithms, instead of spreading the influence of singular forces. In this method, the forcing terms that are a function of the jump conditions and their derivatives are derived from the Taylor expansions rather than approximating the Dirac delta function. This manipulation restricts direct modifications only to the boundary surface. This method was then extended to Stokes flows (LeVeque and Li 1997) and full Navier-Stokes flows (Lee and LeVeque 2003; Li and Lai 2001).

In the implementation of the IIM, the first step is to obtain the singular force acting on the interface. At the early stage, this singular force is calculated as that in the original IB method for elastic boundary or by PID-forcing model for rigid boundary (Goldstein et al. 1993). To avoid numerical instability due to the introduction of some constant parameters in these forcing models, Xu (2012, $2008,2009,2011)$ presented an explicit approach to decompose the singular force into tangential and normal 
components to enforce the prescribed motion of a rigid boundary in an incompressible viscous flow. Le et al. (2006), Tan et al. (2009), and Li et al. (2016) proposed an implicit method to calculate the singular force for the rigid boundary that shared the same basic idea with the direct-forcing scheme. In the work of $\mathrm{Xu}$ and Wang (2008), the singular force is calculated under the spring model or surface tension force model for different problems. Liu et al. (2019) modified the surface tension model to a strictly inextensible model, in which the surface tension was not calculated as an unknown variable but fully satisfied the inextensibility of the boundary surface.

After obtaining singular force acting on the interface, the next step of the IIM is to incorporate this singular force into the jump conditions and relevant derivatives. The relationship between the jump conditions together with their derivatives and the singular forces has been studied by a larger number of studies over recent decades (Lee and LeVeque 2003; LeVeque and Li 1997; Li and Lai 2001). Lai and Li (2001) derived the principal jump conditions across an immersed moving membrane of velocity, pressure, and relevant normal derivatives. $\mathrm{Xu}$ and Wang (2006) systematically derived necessary spatial and temporal jump conditions for simulating viscous flows subject to moving boundaries with the third-order and second-order accuracy near the boundaries. $\mathrm{Xu}$ and Pearson (2015) derived jump conditions for the triangular mesh system in three-dimensional simulations.

Finally, the discretization of governing equations in the vicinity of the immersed object, both inside and outside the interface, is modified implicitly with all these jump conditions and relevant derivatives. During the time advancement, the force term is not enforced only in the advection-diffusion step of the fractional-step scheme, compared with the original IB method. It is divided into tangential component $F_{1}$ and normal component $F_{2}$. The tangential component $F_{1}$ term is involved in the advectiondiffusion step, while the normal component $F_{2}$ that indicates the proper jump condition is solved together with the Poisson equation (Lee and LeVeque 2003) as follows:

Advection-diffusion step:

$$
u_{t}+(u \cdot \nabla) u=\mu \nabla^{2} u+F_{1} ;
$$

Projection step:

$$
U^{n+1}=U^{*}-\Delta t \nabla p^{n+1}+\Delta t F_{2},
$$

$p^{n+1}$ is determined by the Poisson equation

$$
\nabla^{2} p^{n+1}=\frac{1}{\Delta t} \nabla \cdot U^{*}+\nabla \cdot F_{2}
$$

To obtain high-order accuracy, more jump conditions of the variable and its high-order derivatives are favored. Zhong (2007) derived six versions of new IIM up to fourthorder accuracy by using only two jump conditions together with boarder grid stencils. Liang et al. (2008) and Jiang et al. (2012) combined the spectral scheme with the IIM to take advantage of the high accuracy of the spectral method. Hosseinverdi and Fasel (2017) used a compact difference scheme to derive the first- and second-order derivatives with seventh-order and sixth-order accuracy, respectively.

\subsection{Hybrid immersed boundary method}

Besides the aforementioned various strategies, another strategy to improve the versatility of the IB methods is to combine one IB method with the other kind of numerical technique or solver. One successful and famous combination is the coupling of IB method with the lattice Boltzmann method (LBM) (Jahanshaloo et al. 2016). Taking the advantages of IB method in the implementation of boundary conditions and LBM in computational efficiency, the hybrid immersed boundary lattice Boltzmann method (IB-LBM) has become quite popular in studying multiphase flows (Chen et al. 2013; Feng and Michaelides 2004; Suzuki et al. 2018). The classical bounce-back (BB) scheme is usually used in the framework of LBM to handle the fluidparticle interface. Although both the BB scheme and the IB method can obtain an acceptable accuracy for the stationary boundary problem (Chen et al. 2014), the IB method shows better performance on avoiding the fluctuation of the velocity and the body force for a moving boundary (Feng and Michaelides 2004). Moreover, it is easier for the IB method to deal with complex geometries (De Rosis et al. 2014) so that it has demonstrated to be more efficient than the LBM-BB scheme (de Motta et al. 2019).

Combining a specific IB method with another one is also a favorable choice to take advantage of both methods. In the work of Lee and Leveque (2003), the IIM and the original IB method were combined to handle the boundary force in different directions, respectively. Boukharfane et al. (2018) applied the direct-forcing method and the ghost-cell method together to enforce no-slip and nopenetration boundary conditions at the interphase interface in studying compressible reactive flows over immersed objects, respectively. Hu et al. (2018) employed the original IB method to study the moving of an irregular domain while the IIM is used to study the convection- 
diffusion problem at the domain interface. Meyer et al. (2010) coupled the IIM with the cut-cell method to maintain high accuracy, together with a combination of level-set method, which showed excellent mass and momentum conservation for arbitrary moving and deforming boundaries.

Although the IB method was first developed in the framework of the Cartesian grids, it has been successfully extended to the non-Cartesian grid system. Applying advanced grid techniques, such as the adaptive mesh refinement (AMR) technique (Hu et al. 2013; Kedia et al. 2014; Mohapatra et al. 2013; Muralidharan and Menon 2018), the curvilinear grid technique (Nagendra et al. 2014), the overset grid technique (Borazjani et al. 2013), and the local refinement technique (Kang et al. 2009; Pan and Shen 2009), could guarantee the resolution in the core region near the boundary and lower the computational cost in solving the region far away from the boundary. These advanced grid generation techniques work well, especially for high Reynolds number problems and complex geometry problems.

\subsection{Implementation of boundary conditions}

The implementation of different boundary conditions in the framework of IB methods has subtle difference. The enforcement of the Dirichlet boundary condition is the most basic and original part of the IB methods because constructing a no-slip velocity boundary condition is nearly inevitable in multiphase flows. In the original IB method and the direct-forcing method, the no-slip boundary condition gives a constraint to the surface force. Although calculated through different approaches, this surface force generally acts as additional forcing term in governing equations to exert the influence of boundary. In the ghostcell method, the cut-cell method, and the IIM, zero value of the velocity at the interface can be easily specified through interpolating the ghost point values, integrating over the trapezoidal boundary cell, and constructing the jump conditions, respectively. For the Dirichlet boundary condition of temperature, the implementation is similar to the enforcement of the velocity boundary condition.

The construction of the Neumann boundary condition is more complicated than that of the Dirichlet type, especially for a high-order scheme (Barozzi et al. 2004). For this reason, the improvement of IB methods in studying heat transfer problems with higher accuracy is still under study. Kim and Choi (2004) firstly proposed an IB method to handle the Neumann thermal boundary condition, and the energy forcing at the interface was interpolated through the heat flux at the surface and the temperature of the fluid point. Similar work was done by Pacheco et al. (2005) by constructing a Neumann thermal boundary condition with the bilinear-linear interpolation scheme. In the framework of the direct-forcing method, Zhang et al. (2008) computed the temperature at the boundary surface by a first-order extrapolation with the combination of a virtual point in the fluid region and the given heat flux. With the known boundary temperature, the direct-forcing scheme could be applied as straightforward as in the implementation of the Dirichlet temperature boundary condition. In the work of Ren et al. (2013), to enforce the Neumann thermal boundary condition, the energy equation was advanced without considering the thermal effect of IB first. Then in the correction step, the energy source term was calculated based on the difference between the precalculated heat flux and the constant heat flux boundary condition. This idea is similar to the correction of the direct-forcing term and could be termed as a direct-heat method. Wang et al. (2009) developed a multi-direct-forcing and multi-directheat method for the Dirichlet boundary condition. This method has been commented as the best choice for a Dirichlet boundary condition in the framework of IB methods and has further been extended to a Neumann boundary condition by Santarelli (2016). As the main difference of the original IB method and the direct-forcing method is the way to calculate the additional forcing terms and is not relevant to the treatment of thermal conditions, these aforementioned methods could be straightforwardly extended to the original IB method. In the framework of the ghost-cell method, the implementation of the Neumann boundary condition is much easier, as the ghost-cell method is closely related to the interpolation/extrapolation schemes in the calculation of ghost cells. With a slight change in the interpolation scheme to include the derivative term at the interface, the effect of the isoflux boundary condition could be easily integrated, as done in the studies of Luo et al. (2016b), Pan (2012), and Xia et al. (2014).

As for the authentic boundary method, i.e., the IIM and the cut-cell method, the implementation of the Neumann boundary condition is straightforward too. A key point in the IIM and the cut-cell method is to enforce the Neumann boundary condition for pressure to ensure no-penetration at the surface. The given derivatives at the interface could be easily integrated into the calculation of jump conditions and spatial and temporal discretization process for the IIM (Hu et al. 2018; Meyer et al. 2010) or in the calculation of the surface derivatives and values in a finite-volume solver (Schneiders et al. 2013).

The application of enforcing the Robin boundary condition through the IB methods is relatively rare. Pacheco-Vega 
et al. (2007) calculated the temperature at the interphase boundary with iteration and interpolation to fulfill the Robin boundary condition with the direct-forcing method. Pan (2012) and Luo et al. (2016b) independently constructed interpolation schemes to realize the Robin boundary condition in the ghost-cell method with second-order accuracy. It should be noted that the IIM could easily handle the Robintype boundary condition through the derivation of jump conditions and modification to discretization schemes $\mathrm{Hu}$ et al. 2018), which makes the IIM widely applied in complex boundary condition problems.

\subsection{Summary of the IB methods}

All the aforementioned IB methods have their own characteristics. The advantages and defects, implementation difficulty, and scope of application of each method are summarized in Table 1.

Due to the numerical similarity of the original IB method of Peskin (1972) and the direct-forcing method of Uhlmann (2005), summaries of them are presented together. The original IB method and Uhlmann's directforcing method share the same idea of calculating the forces at the surface Lagrangian markers and spreading them to surrounding Eulerian grids by the Dirac delta function. These two methods are easy to understand and implement numerically. Furthermore, pressure boundary conditions are not required at the immersed interface, which can avoid the extra computational cost needed in solving the Poisson equation with additional pressure boundary conditions
(Udaykumar et al. 2001). On one hand, the utilization of the Dirac delta function leads to an artificially finite-size boundary problem. This is critical to high Reynolds number flows, where the turbulent statistics are quite sensitive to the construction of the boundary conditions (Umphrey et al. 2017). On the other hand, the Dirac delta function could smooth the spurious pressure oscillations when handling the moving/deformable boundaries (Seo and Mittal 2011b). This advantage, as well as the numerical simplicity, makes the original IB method and Uhlmann's direct-forcing method suitable for moving/deformable geometries with moderate Reynolds numbers. As for calculating the additional forcing terms, the utilization of elastic forcing models in the original IB method makes it favorable for studying fluid-structure interaction. However, the accuracy of these forcing models greatly relies on empirical parameters. How to choose proper forcing models and corresponding empirical parameters for the original IB method could be sophisticated, while these defects are subtly avoided in the direct-forcing method.

In the ghost-cell method, the effects of the interphase coupling conditions are represented by reconstructing the values at the grid nodes in the vicinity of the embedded body without adding additional terms in the governing equations. The reconstruction scheme and corresponding preliminary manipulation depend on interpolation/ extrapolation so that the ghost-cell method could be easily implemented, and its accuracy could be easily adjusted. However, the requirement of implementing the pressure boundary conditions at the IB could lead to unphysical solutions and more computational cost (Mark and van

Table 1: A summary of five different IB methods.

\begin{tabular}{|c|c|c|c|c|}
\hline $\begin{array}{l}\text { IB method } \\
\text { types }\end{array}$ & Sub-type & $\begin{array}{l}\text { Add } \\
\text { terms }\end{array}$ & Merits & Limitations \\
\hline \multicolumn{5}{|c|}{ Artificial boundary method } \\
\hline & $\begin{array}{l}\text { The original IB } \\
\text { method }\end{array}$ & Yes & $\begin{array}{l}\text { Physically easy. Suitable for fluid-structure } \\
\text { interaction problem. No pressure boundary } \\
\text { condition required. }\end{array}$ & $\begin{array}{l}\text { Relies on empirical parameters. Boundary diffusion } \\
\text { problem. Hard to deal with high Reynolds number and } \\
\text { compressible flow. }\end{array}$ \\
\hline & $\begin{array}{l}\text { Uhlmann's } \\
\text { direct forcing }\end{array}$ & Yes & $\begin{array}{l}\text { Easy to implement. Suitable for moving geom- } \\
\text { etries. No pressure boundary condition } \\
\text { required. }\end{array}$ & $\begin{array}{l}\text { Boundary diffusion problem. Hard to deal with high } \\
\text { Reynolds number and compressible flow. }\end{array}$ \\
\hline & Ghost cell & No & $\begin{array}{l}\text { Physically easy. Easy to implement. Easy to } \\
\text { adjust accuracy. }\end{array}$ & $\begin{array}{l}\text { Hard to implement in moving geometries due to } \\
\text { pressure oscillations. }\end{array}$ \\
\hline \multicolumn{5}{|c|}{ Authentic boundary method } \\
\hline & Cut cell & No & $\begin{array}{l}\text { Sharp boundary representation. Local } \\
\text { conservation. }\end{array}$ & $\begin{array}{l}\text { Hard to implement. Special technique needed to cell } \\
\text { reshaping. Very time-consuming in three- } \\
\text { dimensional/moving geometries. }\end{array}$ \\
\hline & IIM & No & $\begin{array}{l}\text { Sharp boundary representation. Volume con- } \\
\text { servation. Suitable for complex boundary } \\
\text { condition. }\end{array}$ & $\begin{array}{l}\text { Sophisticated to deduce jump conditions for each } \\
\text { application. }\end{array}$ \\
\hline
\end{tabular}


Wachem 2008). Besides, pressure oscillations would occur in the vicinity of the boundary in handling moving boundary due to some potential sources, such as the "fresh cell" and "dead cell," the pressure boundary condition, and the nonconservation. Efforts should be made in the future to overcome these issues for the ghost-cell method.

The cut-cell method is usually incorporated with the finite-volume solver. It reshapes the cells near the boundary by emerging and splitting and then exerts the boundary conditions by specifying boundary conditions at the cell surface without additional terms in the governing equations. The application of the finite-volume solver and reshaping scheme enables an authentic boundary representation and local conservation. However, difficulty in implementing a cut-cell method is mainly about the reshaping scheme. As discussed earlier, dealing with the extreme small cells and complex geometries is inevitable in the realization of the cut-cell method. The extension of the cut-cell method from the two-dimensional problem to three-dimensional would also pose a significant burden in computational cost. In particular, the reshaping of cut cells needs to be carried out during every time step in handling moving boundaries, which is also very time-consuming. More efficient techniques are desired.

As for the IIM, the interphase coupling conditions are quantified as jump conditions at the boundary surface and then incorporated into temporal advancement and spatial discretization without explicitly modifying the governing equations. As a result, the strict capturing of the boundary interface and the local conservation could be guaranteed. However, deriving a correct jump condition is somewhat sophisticated. For a new configuration, a new set of singular forces and jump conditions are usually required. This procedure will be more sophisticated in handling moving boundaries because these numerical manipulations should be carried out during every time step.

It is of great interest to quantitatively compare and evaluate each IB method based on some benchmark cases; however, a direct comparison of each IB method is quite scarce in literature, and most of them are limited to the direct-forcing method and the ghost-cell method. In the work of Kang and Hassan (2010, 2011), the performance of the multi-direct-forcing method and the ghost-cell method is compared in simulations of flow past stationary and moving boundaries. For a stationary boundary, the ghostcell method works better than the multi-direct-forcing method. While for moving boundary, the ghost-cell method showed spurious oscillations in pressure field and surface force coefficients. It was concluded that with a proper radius correction, the multi-direct-forcing method (Luo et al. 2007b; Wang et al. 2008) could behave better than the ghost-cell method in both stationary and moving problems. Delouei et al. (2015) studied a thermal flow past a heated cylinder with the direct-forcing method and the ghost-cell method. It was pointed out that the ghost-cell method was better than the direct-forcing method in computational cost and accuracy but harder in implementation for a stationary thermal boundary problem in a steady regime. Lu et al. (2019b) studied the forced convection of flow past particle systems in a tube using both the direct-forcing method and the high-order ghost-cell method. Although they found out that the computational costs for these two methods were almost the same, the direct-forcing method would introduce inaccuracy in capturing the heat transfer coefficient due to the usage of the Dirac delta function. Santarelli et al. (2016) compared the multi-direct-forcing method (Tavassoli et al. 2013; Wang et al. 2009) with the ghost-cell method in simulations of flow past a stationary sphere under isothermal, isoflux, and adiabatic thermal boundary conditions. They found that for the isothermal case, the ghost-cell method showed worse accuracy. While for the isoflux and adiabatic cases, the ghost-cell method performed better. However, rigorous comparisons and general conclusions are still on the way.

\section{Applications of IB methods to multiphase transport phenomena}

With the development of the IB methods and the computer capability, fully resolved simulations of multiphase flows with heat and mass transfer problems have gained more and more attention, and significant progresses have been achieved. In this section, we will give an overview of recent applications of the IB methods in multiphase transport phenomena, including isothermal flows, multiphase heat transfer problems, and mass transfer problems, with particular focus on the latter two topics.

\subsection{Isothermal flows}

Particle-laden isothermal flows exist in many industrial processes, such as suspension and circulating of particles in fluidized beds, transportation of powder, and vaporization of spray in combustors, and so on. This kind of flow has attracted many studies to develop advanced drag model to predict dispersed particle motion. Configurations studied with the IB methods in this topic mainly include 
uniform flow past a stationary object problem, moving problem with simple geometry, and multiobject systems such as flow past a series of particles and fluidized bed system.

The configuration of a uniform flow past a stationary object, especially the uniform flow past a two-dimensional circular cylinder (Choi et al. 2007; Kim et al. 2001; Lai and Peskin 2000; Le et al. 2008; Linnick and Fasel 2005; Saiki and Biringen 1996; Silva et al. 2003; Tseng and Ferziger 2003; Uhlmann 2005; Ye et al. 1999; Zhang and Zheng 2007) or a three-dimensional sphere (Choi et al. 2007; Gao et al. 2007; Kim et al. 2001; Le et al. 2008; Zhang and Zheng 2007) immersed in a fluid stream, has been considered as a benchmark for numerical validation of various IB methods. A summary of the characteristic parameters and simulation configurations from previous studies is presented in Tables 2 and 3, where the column "Grid resolution" stands for how many grid points lie within a cylinder/sphere diameter. All the aforementioned five subtypes of IB methods are covered in these studies. In this simple configuration, different kinds of flow behaviors can be captured depending on the Reynolds number, which varies from 20 to 300 . With the increase of the Reynolds number, the flow pattern would change from a steady state with symmetrical standing vortices to unsteady vortex shedding state. In the study of flow past a

Table 2: Summary of simulation setup and flow parameters for uniform flow past a two-dimensional cylinder.

\begin{tabular}{|c|c|c|c|c|c|c|c|}
\hline References & Method & $\begin{array}{r}\text { Grid } \\
\text { resolution }\end{array}$ & $\begin{array}{l}\text { Reynolds } \\
\text { number }\end{array}$ & Flow pattern & $\begin{array}{l}\text { Drag force } \\
\text { coefficient }\end{array}$ & $\begin{array}{r}\text { Lift force } \\
\text { coefficient }\end{array}$ & $\begin{array}{r}\text { Strouhal } \\
\text { number }\end{array}$ \\
\hline Lai and Peskin (2000) & $\begin{array}{l}\text { The original IB } \\
\text { method }\end{array}$ & 38.4 & $\begin{array}{r}{[100,150,} \\
200]\end{array}$ & $\begin{array}{l}\text { Vortex } \\
\text { shedding }\end{array}$ & {$[1.4473,-,-]$} & {$[0.3299,-,-]$} & $\begin{array}{r}{[0.166,0.183} \\
0.197]\end{array}$ \\
\hline Uhlmann (2005) & Direct forcing & 38.4 & 100 & $\begin{array}{l}\text { Vortex } \\
\text { shedding }\end{array}$ & 1.501 & 0.349 & 0.172 \\
\hline Ye et al. (1999) & Cut cell & - & $\begin{array}{r}{[20,40]} \\
{[80,300]}\end{array}$ & $\begin{array}{l}\text { Steady } \\
\text { Vortex } \\
\text { shedding }\end{array}$ & $\begin{array}{l}{[2.03,1.52]} \\
{[1.37,1.38]}\end{array}$ & - & {$[0.15,0.21]$} \\
\hline $\begin{array}{l}\text { Linnick and Fassel } \\
(2005)\end{array}$ & IIM & - & $\begin{array}{r}{[20,40]} \\
{[100,200]}\end{array}$ & $\begin{array}{l}\text { Steady } \\
\text { Vortex } \\
\text { shedding }\end{array}$ & $\begin{array}{l}{[2.06,1.54]} \\
{[1.34,1.34]}\end{array}$ & {$[0.333,0.69]$} & {$[0.166,0.197]$} \\
\hline Kim et al. (2001) & Ghost cell & 30 & $\begin{array}{r}40 \\
100\end{array}$ & $\begin{array}{l}\text { Steady } \\
\text { Vortex } \\
\text { shedding }\end{array}$ & $\begin{array}{l}1.51 \\
1.33\end{array}$ & $\begin{array}{r}- \\
0.32\end{array}$ & 0.165 \\
\hline $\begin{array}{l}\text { Saiki and Biringen } \\
\text { (1996) }\end{array}$ & $\begin{array}{l}\text { The original IB } \\
\text { method }\end{array}$ & - & $\begin{array}{r}{[25,30]} \\
{[50,65,100]}\end{array}$ & $\begin{array}{l}\text { Steady } \\
\text { Vortex } \\
\text { shedding }\end{array}$ & $\begin{array}{r}{[1.54,1.38]} \\
{[1.38,1.33,} \\
1.26]\end{array}$ & - & {$[0.139,0.152$} \\
\hline & & & {$[200,400]$} & $\begin{array}{l}\text { Vortex } \\
\text { shedding }\end{array}$ & {$[1.18,1.18]$} & - & {$[0.197,0.22]$} \\
\hline Silva et al. (2003) & $\begin{array}{l}\text { The original IB } \\
\text { method }\end{array}$ & 16.7 & {$[10,20,40]$} & Steady & $\begin{array}{r}{[2.81,2.04} \\
1.54]\end{array}$ & - & \\
\hline & & & {$[47,50,80]$} & $\begin{array}{l}\text { Vortex } \\
\text { shedding }\end{array}$ & $\begin{array}{r}{[1.46,1.46} \\
1.40]\end{array}$ & - & $\begin{array}{r}{[0.11,0.121} \\
0.15]\end{array}$ \\
\hline & & & $\begin{array}{r}{[100,150} \\
300]\end{array}$ & $\begin{array}{l}\text { Vortex } \\
\text { shedding }\end{array}$ & $\begin{array}{r}{[1.39,1.37,} \\
1.27]\end{array}$ & - & $\begin{array}{r}{[0.161,0.181} \\
0.20]\end{array}$ \\
\hline Tseng and Ferziger & Ghost cell & - & 40 & Steady & 1.53 & - & - \\
\hline$(2003)$ & & & 100 & $\begin{array}{l}\text { Vortex } \\
\text { shedding }\end{array}$ & 1.42 & 0.29 & 0.164 \\
\hline Choi et al. (2007) & Ghost cell & $\begin{array}{l}41 \\
81\end{array}$ & $\begin{array}{r}{[20,40]} \\
{[100,200]}\end{array}$ & $\begin{array}{l}\text { Steady } \\
\text { Vortex } \\
\text { shedding }\end{array}$ & $\begin{array}{l}{[2.02,1.52]} \\
{[1.34,1.36]}\end{array}$ & {$[0.315,0.64]$} & {$[0.164,0.191]$} \\
\hline $\begin{array}{l}\text { Zhang and Zheng } \\
\text { (2007) }\end{array}$ & Direct forcing & 40 & $40-550$ & - & - & - & - \\
\hline \multirow[t]{2}{*}{ Le et al. (2008) } & IIM & - & $\begin{array}{r}{[20,40]} \\
{[80,100]}\end{array}$ & $\begin{array}{l}\text { Steady } \\
\text { Vortex } \\
\text { shedding }\end{array}$ & $\begin{array}{l}{[2.07,1.58]} \\
{[1.42,1.39]}\end{array}$ & {$[0.257,0.346]$} & {$[0.15,0.16]$} \\
\hline & & & {$[200,300]$} & $\begin{array}{l}\text { Vortex } \\
\text { shedding }\end{array}$ & {$[1.38,-]$} & {$[0.676,-]$} & {$[0.192,0.208]$} \\
\hline
\end{tabular}


Table 3: Summary of simulation setup and flow parameters for uniform flow past a three-dimensional sphere.

\begin{tabular}{|c|c|c|c|c|c|c|c|}
\hline References & Method & $\begin{array}{r}\text { Grid } \\
\text { resolution }\end{array}$ & $\begin{array}{c}\text { Reynolds } \\
\text { number }\end{array}$ & Flow pattern & $\begin{array}{l}\text { Drag force } \\
\text { coefficient }\end{array}$ & $\begin{array}{l}\text { Lift force } \\
\text { coefficient }\end{array}$ & $\begin{array}{c}\text { Strouhal } \\
\text { number }\end{array}$ \\
\hline \multirow[t]{3}{*}{ Kim et al. (2001) } & Ghost cell & - & 100 & Steady axisymmetric & 1.087 & - & - \\
\hline & & & 250 & Steady asymmetric & 0.701 & 0.059 & - \\
\hline & & & 300 & Unsteady & 0.657 & 0.067 & 0.134 \\
\hline \multirow[t]{3}{*}{ Choi et al. (2007) } & Ghost cell & 41 & 100 & Steady axisymmetric & 1.09 & - & - \\
\hline & & 81 & 250 & Steady asymmetric & 0.7 & 0.052 & - \\
\hline & & 81 & 300 & Unsteady & 0.658 & 0.068 & 0.134 \\
\hline Zhang and Zheng (2007) & Direct forcing & 20 & $30-100$ & Steady axisymmetric & - & - & - \\
\hline \multirow[t]{4}{*}{ Le et al. (2008) } & IIM & - & {$[50,100]$} & Steady axisymmetric & {$[1.66,1.16]$} & - & - \\
\hline & & & {$[150,200]$} & Steady axisymmetric & {$[0.96,0.85]$} & - & - \\
\hline & & & 250 & Steady asymmetric & - & 0.057 & - \\
\hline & & & 300 & Unsteady & 0.685 & - & - \\
\hline \multirow[t]{2}{*}{ Gao et al. (2007) } & Ghost cell & 33.3 & 100 & Steady axisymmetric & 1.09 & - & - \\
\hline & & & 300 & Unsteady & 0.66 & - & - \\
\hline
\end{tabular}

two-dimensional cylinder, the critical particle Reynolds number for the transition to unsteady vortex shedding is at $R e=47$ (Silva et al. 2003). However, the evolution of the flow pattern becomes more complicated in the flow past a three-dimensional spherical particle. With the enhancement of the Reynolds number, three different flow regimes exist: steady axisymmetric regime ( $R e<200)$, steady asymmetric regime $(210<R e<270)$, and unsteady flow regime ( $R e>280$ ) (Choi et al. 2007). Besides validation, the linear stability analysis of flow past bluff bodies with simple geometries by using the IB methods has also drawn much attention in the recent years. The instability mechanisms for the two-dimensional flow past a single cylinder (Giannetti and Luchini 2007) or two side-by-side cylinders (Carini et al. 2014) and three-dimensional flow past a rotating circular cylinder (Pralits et al. 2013) have been investigated. The instability of thermal-driven natural convection problems has been explored too (Feldman 2018a; Feldman and Gulberg 2016).

Although the Reynolds numbers of particles in chemical and other industrial applications are often small, it is not always reasonable to assume the particles to be stationary, especially in the case where particle transport and mixing are of primary interest. For moving boundary problems with simple geometries, two kinds of problems have been well studied. One is the flow past a periodic inline or transversely oscillating cylinder or sphere (Choi et al. 2007; Gilmanov and Sotiropoulos 2005; Kumar and Roy 2016; Lee et al. 2011; Liao et al. 2010; Liao and Lin 2012b; Luo et al. 2010, 2012; Schneiders et al. 2013; Su et al. 2007; Yang and Balaras 2006). During the oscillation of cylinder, the vortex shedding "lock-in" phenomenon may occur when the frequency of vortex shedding in the wake synchronizes with the frequency of an imposed perturbation at the cylinder, as shown in Figure 5A. This phenomenon would lead to oscillations of flow patterns and enhancement of drag and lift coefficients. The other is the free sedimentation of particles (Delouei et al. 2016; Kempe and Fröhlich 2012; Kim and Choi 2006; Lo et al. 2018; Luo et al. 2007a; Luo et al. 2013; Suzuki et al. 2018; Yang and Stern 2013). One of the most well-known phenomena in particle sedimentation is the "drafting, kissing and tumbling" phenomenon, or called as DKT motion, as shown in Figure 5B. This phenomenon occurs when a trailing particle follows the wake of the leading particle, the trailing particle will accelerate and contact with the leading particle due to the low pressure in the wake region. These two unique microscale phenomena have been widely studied for validation of the IB methods in handling the moving boundary problem. In addition, the capability of IB method in handling deformable boundary has enabled its applications to biomedical domain, such as proliferation and fusion of cells (Rejniak et al. 2004), growth and development patterns of tumor microarchitectures (Rejniak 2007; Rejniak and Dillon 2007).

However, in realistic applications of the industrial process, a large number of particles are usually involved. In many-particle systems, the particle-particle interactions, including collisions and indirect mutual effects, would substantially alter the flow patterns and particle hydrodynamics compared with the single-particle case. Applying the IB methods to study these effects and develop better drag force models for many-particle system is of great interest.

Tenneti et al. (2011) studied the flow past fixed random assemblies of monodisperse spheres at a Reynolds number up to 300 by using the PUReIBM. A new 
drag correlation, fitting well with the comprehensive set of drag data from the monodisperse gas-solid suspensions, was proposed for a Reynolds number up to 300 and a solid volume fraction $\phi$ in the range of $0.1-0.5$ as follows

$$
\begin{aligned}
F_{d}(\phi, \operatorname{Re})= & \frac{1+0.15 R e^{0.687}}{(1-\phi)^{3}} \\
& +(1-\phi)^{2}(1+1.5 \sqrt{\phi})+\frac{5.81 \phi}{(1-\phi)^{3}} \\
& +0.48 \frac{\phi^{1 / 3}}{(1-\phi)^{4}}+\phi^{3} \operatorname{Re}\left(0.95+\frac{0.61 \phi^{3}}{(1-\phi)^{2}}\right) .
\end{aligned}
$$

They concluded that special attention should be paid to the grid resolution. With the increase of solid volume fraction from 0.2 to 0.4 , the allowable minimum grid resolution is also enhanced from 40 to 60 . This is a very severe burden for computational resources in simulations of large-scale particle system. Following this work, Mehrabadi et al. (2016) studied the drag force on particle clusters with a solid volume fraction $\phi$ ranging from 0.1 to 0.5 and a Reynolds number ranging from 0.01 to 50 . It was found that the drag experienced by particle clusters was reduced compared with those for uniform particles, especially in low solid volume fraction case.

Tang et al. (2014) studied flow past stationary assemblies of 108 monodisperse spheres in random configurations with the Reynolds number at 50 and 100 and a solid volume fraction $\phi$ ranging from 0.1 to 0.6 by using an iterative direct-forcing IB method. Correlations for the drag force coefficient were proposed for these two Reynolds numbers. In their work, the grid resolution was chosen to be around 16. Later they (Tang et al. 2015) extended the study to cover the Reynolds number of 50-1000 and then proposed a new correlation for interphase force as follows

$$
\begin{aligned}
F_{d}(\phi, R e)= & \frac{10 \phi}{(1-\phi)^{2}}+(1-\phi)^{2}(1+1.5 \sqrt{\phi}) \\
& +\left[0.11 \phi(1+\phi)-\frac{0.00456}{(1-\phi)^{4}}\right. \\
& \left.+\left(0.169(1-\phi)+\frac{0.0644}{(1-\phi)^{4}}\right) R e^{-0.343}\right] R e .
\end{aligned}
$$

Zaidi et al. (2014) applied the direct-forcing IB method to investigate the drag force of flow past randomly placed spheres moving at the same velocity. The solid volume fraction and Reynolds number were varied from 0.05 to 0.5 and 0.01 to 1000 , respectively. The grid resolution ranged from 17 to 26 . A drag law was developed as

$$
F_{d}=\left\{\begin{array}{r}
\frac{10 \phi}{(1-\phi)^{3}}+(1-\phi)(1+1.5 \sqrt{\phi}) \\
+\frac{0.034}{(1-\phi)^{3.7}} \operatorname{Re}(\operatorname{Re} \leq 200) . \\
\frac{10.9 \phi^{0.4}}{(1-\phi)^{2.7}}+\frac{0.024}{(1-\phi)^{3.86}} \operatorname{Re}(\operatorname{Re}>200)
\end{array}\right.
$$

In the study of Uhlmann and Doychev (2014), the gravity-induced sediment of particles under dilute conditions was investigated by using the direct-forcing IB method. The average particle settling velocity of the clusters was increased by $12 \%$ compared with that of the single particle, which was associated with the downward fluid motion induced by the clustering. The variance of the particle velocity was also higher because of the fluid velocity fluctuation. The particles with higher Galileo number (a nondimensional number proportional to gravity forces divided by viscous forces) tended to form the vertically elongated columnar regions, while the particles with lower Galileo number showed slightly more ordered than a random Poisson distribution. In the study of Lashgari et al. (2014), three flow regimes were identified based on the Reynolds number and the particle volume fraction. Except for the laminar and the turbulent regimes, a new regime was found when the volume fraction was sufficiently large (greater than 0.1). In this new regime, the wall friction was even higher than that in the turbulent regime. The increase of the wall friction was not associated with the increase of the Reynolds number (i.e. turbulent transport), and the stress induced by the particles also played an important role. Meanwhile, due to the suspension of particles, the mean streamwise velocity profiles were altered, and the von Kármán constant in the log-law decreased (Picano et al. 2015).

Kriebitzsch et al. (2013) performed fully resolved simulation of a gas-fluidized bed laden with 2000 particles by using the combined direct-forcing IB method and the hard-sphere model. It was found that the averaged fluidparticle force from existing discrete element simulation is $33 \%$ smaller than that from the fully resolved simulation. They believed that the drag correlation should consider the effects of fluctuations of particle positions, velocities, and granular temperature to achieve more accurate DEM simulations. Tang et al. (2016) simulated a gas-fluidized bed loaded with 5000 sphere particles by using the directforcing IB method. Detailed comparison between the fully resolved DNS results and the experimental data from a laboratory gas fluidized bed was firstly reported, and a good agreement was obtained. It was emphasized that 


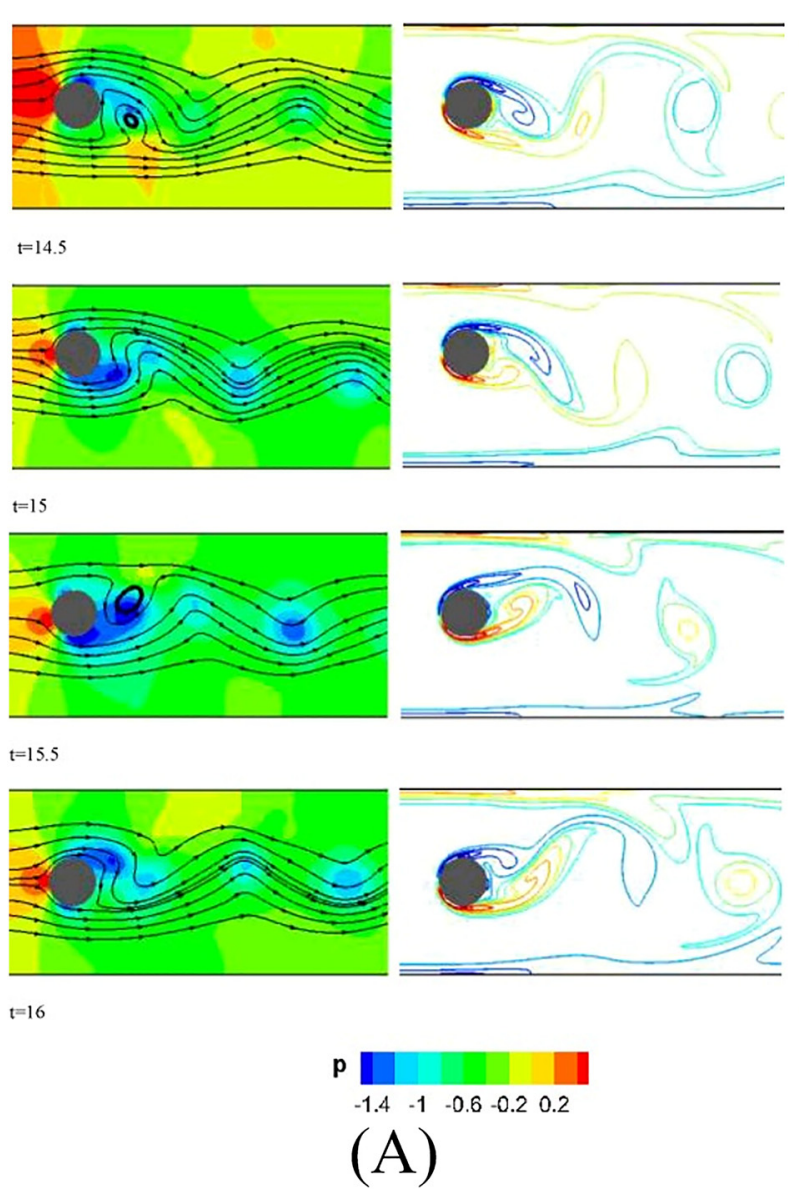

more detailed information could be achieved than traditional experimental studies and non-fully-resolved simulations, such as granular temperature.

Luo et al. (2016a) simulated a laboratory bubbling fluidized bed loaded with 9240 particles with the multidirect-forcing IB method. To the best of the authors' knowledge, this is the largest-scale fully resolved simulation of laboratory fluidized beds, which covers the maximum number of particles. The $\lambda$ vortex structures (Jeong and Hussain 1995) in the central slice of the bubbling fluidized bed are shown in Figure 6, together with the particles. It can be seen that numerous small vortices appear in the bottom of bubbling fluidized bed and isolated wake vortices appear around each particle in the bubble growth area, whereas nearly no vortices exist in the nearwall region. The time-averaged particle vertical velocities showed a better agreement with the experimental data than the classic DEM results. The analysis of particle drag force demonstrated that the prevailing DEM drag force models underestimate the particle drag force in fluidized beds, which is consistent with the study of Kriebitzsch et al. (2013).

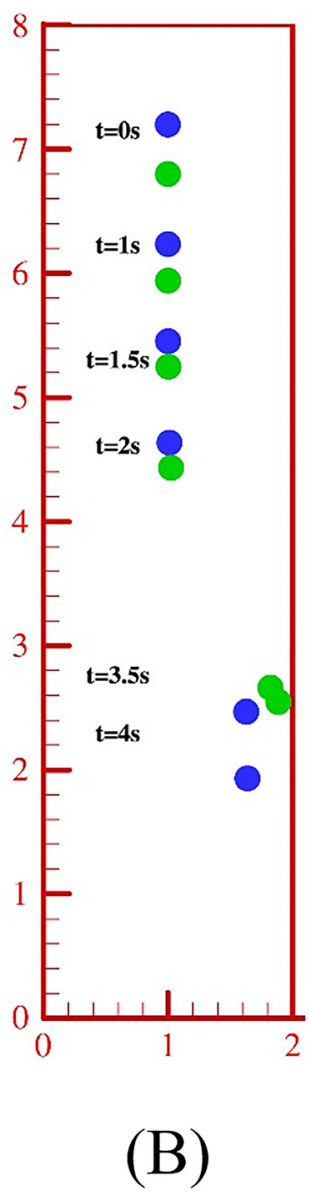

Figure 5: (A) Pressure contour along with streamline (left) and vorticity (right) for transversely oscillating cylinder at $R e=100$ in a channel at different time instants. (B) Sedimentation of two cylindrical particles in a channel at different time stages. Reprinted from Kumar and Roy (2016) and Delouei et al. (2016) respectively with permission of Elsevier.
To summarize, fully resolved simulations of multiphase isothermal flows for a single object with simple geometry at low Reynolds numbers are abundant in the literature. However, challenges appear in studying large-scale system loaded with many particles and high Reynolds number by using the IB methods. With the increase of particle number and solid volume fraction, the grid resolution and computational cost emerge as important issues (Tenneti et al. 2011). More attention should be paid to get grid-independent results from the fully resolved simulations. What is more, existing studies of particle drag correlation were mainly based on the assumption of stationary particles, which is quite unrealistic. The drag coefficient analysis in fully resolved simulation of movable particles in fluidized beds showed that the drag correlation coefficients are remarkably larger than those from the traditional prevailing drag models (Luo et al. 2016a; Tang et al. 2016). This needs to be confirmed by more studies. A more universal and accurate drag force model should also be developed for dense flows, for which an effort has been made recently (Luo et al. 2020). 


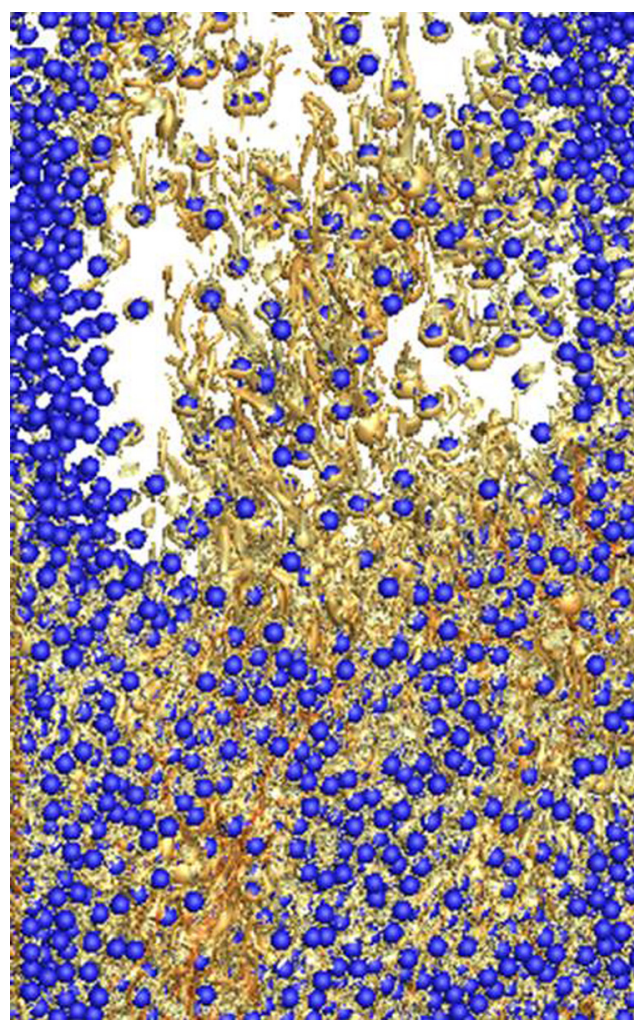

Figure 6: The lambda vortex structure (yellow isosurfaces) in the bubbling fluidized bed. Particles are represented by blue spheres. Reprinted from Luo et al. (2016a) with the permission of John Wiley and Sons.

\subsection{Heat transfer problems}

Heat transfer in multiphase flow is a crucial problem in the design of gas-solid contactors, such as packed beds and fluidized beds. Having a better understanding of the interphase heat exchange process could shed some light on increasing heat exchange efficiency and enhancing energy utilization in chemical systems. For most of the cases we consider here, the heat transfer problems in multiphase flows could be divided into three types, namely the natural convection, the forced convection, and the conjugate heat transfer. Natural convection is a phenomenon of heat transport in the flow without external driving. While in contrast, forced convection is accompanied by externally driven fluid motion. Conjugate heat transfer refers to the coupled heat transfer process considering both convection heat transfer in the fluid side and conduction heat transfer in the solid side.

\subsubsection{Natural convection}

There are two major configurations in the investigation of the natural convection process by using the IB methods.
One is the natural convection within an enclosure, and the other is the sedimentation process accompanied by natural convection.

Natural convection within an enclosure is substantial to many industrial and environmental applications such as heat exchangers, chemical reactors, cooling of electronic equipment, and refrigerator condensers. Due to the exclusion of external flow motion, the Rayleigh number is demonstrated to be a dominating factor in characterizing flow and thermal patterns and distribution of local Nusselt number. Kim et al. (2008) studied two-dimensional natural convection of a heated cylinder within a cold square enclosure by using the ghost-cell method. When the Rayleigh number was small at $R a=10^{3}$ and $10^{4}$, the thermal field was fully symmetric, and four counterrotating vortices occurred, as shown in Figure 7. In the case with a stronger buoyancy effect, such as $R a=10^{5}$ and $10^{6}$, the intense heat convection generated a thermal plume, which modified the flow field significantly, and only two recirculating eddy cores existed so that the dominant flow became upward. The thermal boundary layer at the cylinder surface also became thinner due to the strong convection. Similar phenomena were also observed in many other studies with different complex geometries (Liao and Lin 2012a; Park et al. 2013a; Park et al. 2013b).

Recently, natural convection in a three-dimensional cubic enclosure immersed with cylinder has received much attention (Feldman 2018b; Lee et al. 2016; Seo et al. 2016; Spizzichino et al. 2019b). Comparing with the twodimensional calculations, significant difference exists for the thermal and flow fields (Choi et al. 2015b; Pandey et al. 2019) as well as the surface-averaged Nusselt number (Choi et al. 2015a) in the three-dimensional simulations, even the overall computational parameters are similar. One typical and excellent three-dimensional study has been done by Seo et al. (2019a; 2019b). They demonstrated that the $\lambda$ vortex structures (Jeong and Hussain 1995) in the natural convection of circular cylinder in a long rectangular enclosure are evidently three-dimensional and much more complicated than those in the two-dimensional cases, as shown in Figure 8.

One interesting and notable feature of the natural convection within an enclosure is the unsteadiness. For an enclosure with simple symmetric bifurcated geometry, the flow field and thermal field are generally symmetrically bifurcated too. If the buoyancy effect is strong enough, even though the geometries of embedded object and enclosure are symmetric, the flow and thermal patterns could be asymmetric and unsteady (Cho et al. 2019; Cho et al. 2018; Choi et al. 2014). The asymmetric distribution of flow and thermal fields also leads to an asymmetric distribution of 


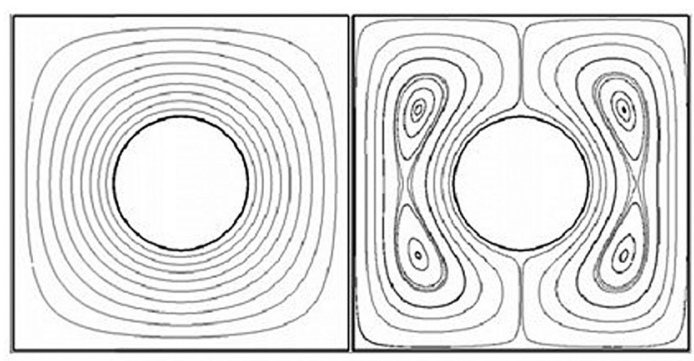

(A) $R a=10^{3}$

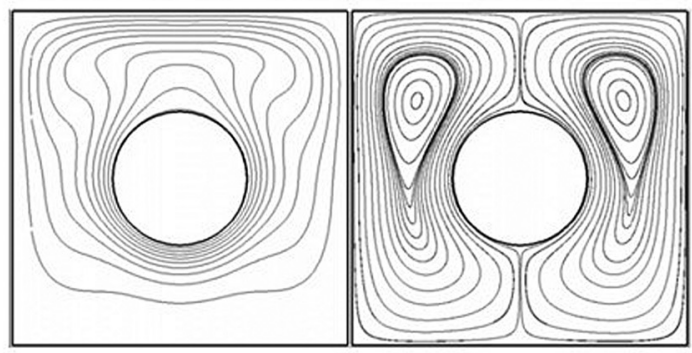

(C) $R a=10^{5}$

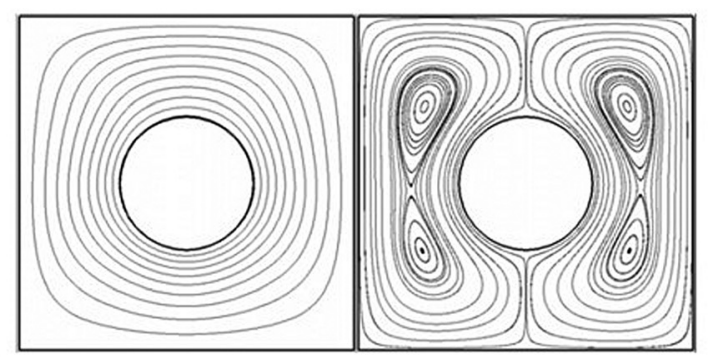

(B) $R a=10^{4}$

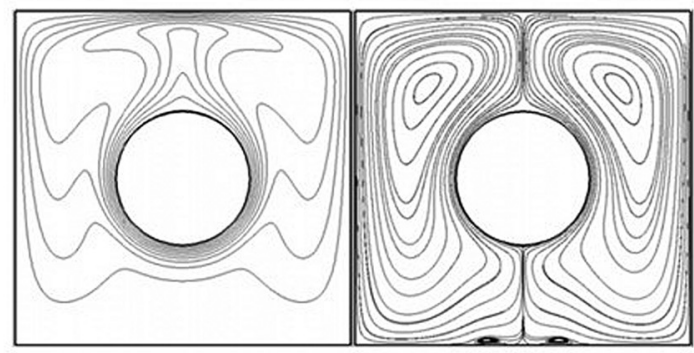

(D) $R a=10^{6}$

Figure 7: Isothermals (left) and streamlines (right) at four different Rayleigh numbers for the natural convection of a circular cylinder at the center within a square enclosure. Reprinted from Kim et al. (2008) with permission of Elsevier.

local Nusselt number at the enclosure surface due to the domination of convection (Park et al. 2013b). However, currently there is still no quantitative indicator for estimating the unsteadiness of flow and thermal fields, as this feature is influenced by geometries of enclosure and interior object, thermal boundary conditions, relative position, inclination angle, and aspect ratio of the embedded object, as well as primarily the Rayleigh number.

The other frequently studied problems are the sedimentation process of particles with natural convection. In these cases, the thermal flux between particles and fluid would modify the flow fields around particles under the influence of buoyancy force and then alter the motion of the particles. This combination effects would result in a huge change of particle settling speed compared with that without heat transfer or buoyancy effect. In the work of $\mathrm{Wu}$ et al. (2017), sedimentations of a hot, cold, and isothermal single particle in an infinite channel were studied by using the hybrid IB-LBM. Heat transfer of particle to fluid was proved to influence the buoyancy effect significantly, thus making the trailing vortices of hot settling particle disappear, as shown in Figure 9. It was reported that the hot particle owned the lowest settling velocity, which was only $50 \%$ of that in the isothermal case. While for cooled particle, the settling velocity is $40 \%$ more than that of the isothermal case. Xia et al. (2015) applied the high-order ghost-cell method to study the sedimentation of a hot spherical particle in the cool fluid. It was found out that for all the Reynolds number considered ( $R e=50,71$, and 100), particle settling velocity considering buoyancy force (Grashof number $G r=5000$ ) was much smaller compared

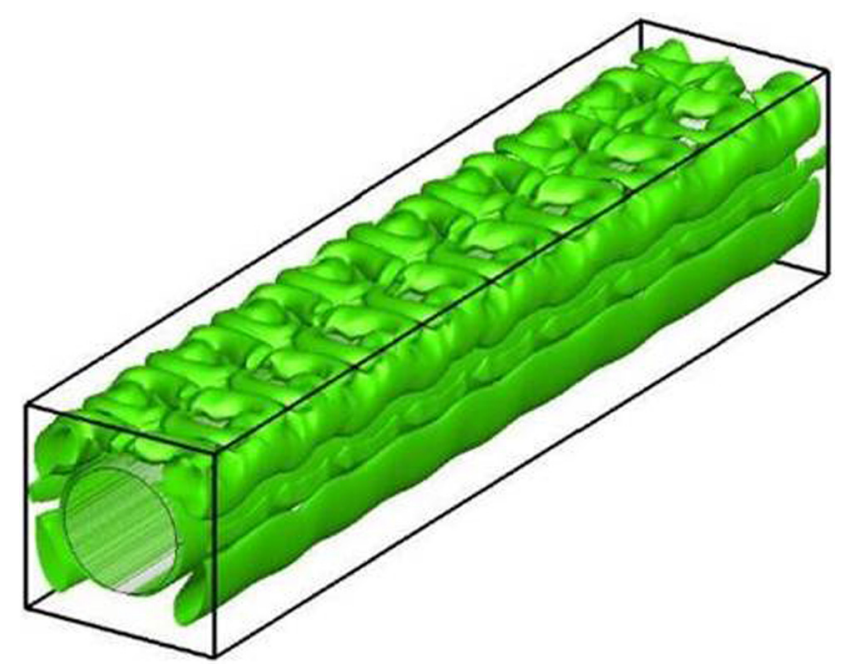

Figure 8: The lambda vortex structure (green isosurfaces) in the natural convection of circular cylinder in a long rectangular enclosure at $R a=10^{6}$. Reprinted from Seo et al. (2019a) with the permission of Elsevier. 


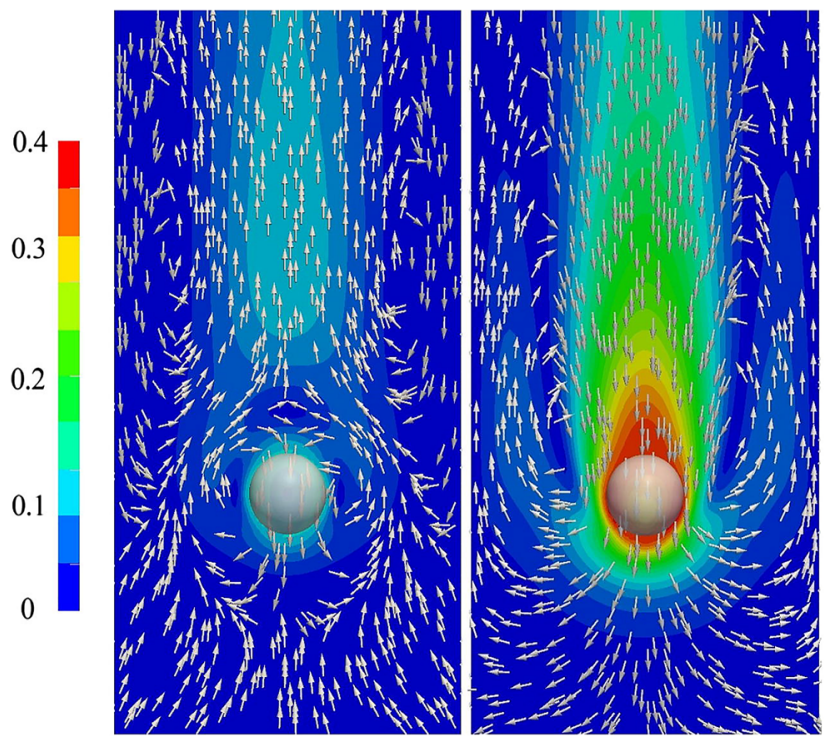

Figure 9: Dimensionless velocity fields of the fluid in the sedimentation of single hot particle (left) and cold particle (right) cases at $R e=40.5$. The contours and the vectors represent the magnitude and the direction, respectively. Particles are represented by white spheres. Reprinted from Wu et al. (2017) with the permission of Elsevier.

with those cases without buoyancy force $(\mathrm{Gr}=0)$ at the same Reynolds number. The heat transfer and hydrodynamics interaction were strongest in the case of $R e=50$ and $G r=5000$, which formed the highest Richardson number in their simulations.

Sedimentation of particle clusters has great importance in industrial applications. Compared with single-particle settling, sedimentation of clusters of particles becomes more complicated due to particle-particle interaction and hydrodynamics-thermal interaction. Feng and Michaelides (2009) studied the settling and cooling of 56 particles with the direct-forcing IB method. The particle-fluid density ratio was set as 1.5, Prandtl number as $\operatorname{Pr}=1$, Grashof number as $G r=200$. It was pointed out that there exists strong interaction between particle trajectory and heat transfer, and the thermal wake of leading particles could help the following particles keep higher temperatures.

Musong et al. (2015) studied the sedimentation of vertically and horizontally placed bispheres and triangular clusters at $\mathrm{Pr}=0.72$ and $\mathrm{Gr}=100$ with the direct-forcing method. They concluded that the orientation and distance between particles significantly affected the average Nusselt number and temperature patterns. For in-line placed two particles system, increasing the distance between particles would always increase the average heat transfer rate. For tangentially placed two particles system, the increase of distance between each particle would initially lead to an enhanced heat transfer rate. Further increasing would make the combined plume structure isolated, and the bispheres act like two single spheres. For multiple particles system, the average heat transfer rate per sphere particle always decreased with the increase of the number of spherical particles in a cluster.

Eshghinejadfard and Thévenin (2016) investigated the movements of 60 spherical particles under natural convection with a low solid-fluid density ratio of 1.1 at $P r=0.7$ and $\mathrm{Gr}=1000$ by using the direct-forcing IB-LBM. Particles were released at the bottom of the computational domain and firstly settled down and then quickly rose up to the top of the enclosure, as shown in Figure 10. Particle-particle and particle-fluid interactions generated strong vortical structures and made some individual particles move upward quicker than the single-particle case. Sometimes this strong vortex could even drive the particles to move back to the bottom of the enclosure.

In summary, multiphase flow with natural convection has been well studied using the IB methods. However, a systematic understanding of natural convection is still on the way. There is no criterion for determining whether the flow could eventually reach a steady state for the natural convection within an enclosure. Further investigations are required to explain the mechanism for the threedimensional unsteadiness in natural convection within an enclosure. Also, the multiparticle sedimentation with heat transfer is far from clear. The distinct particle behaviors
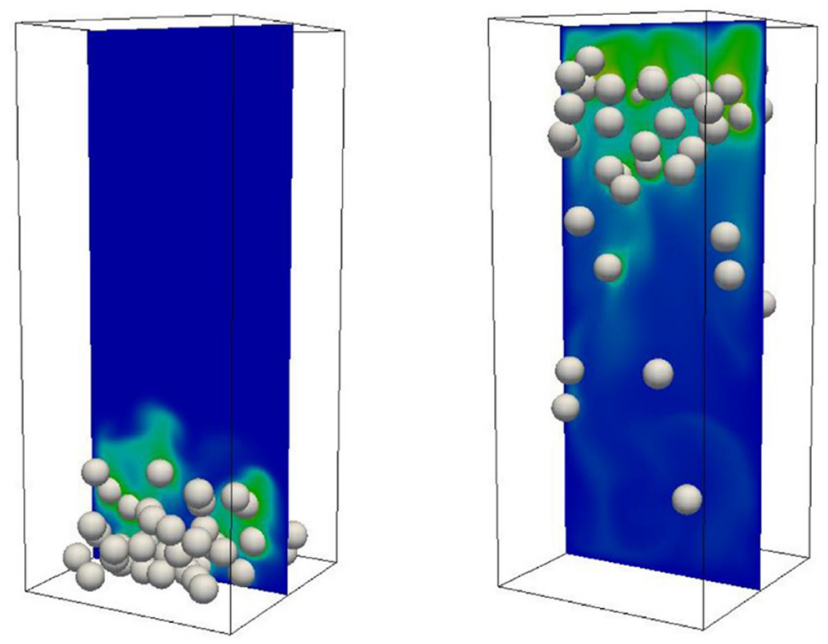

Figure 10: Temperature distribution of the center plane and particle position evolution at different times due to the natural convection in the enclosure; red and blue represent the higher and lower temperatures, respectively. Particles are represented by white spheres. Reprinted from Eshghinejadfard and Thévenin (2016) with permission of Elsevier. 
in the multiparticle system due to buoyancy force need further investigations (Eshghinejadfard and Thévenin 2016). Mechanism studies of the interaction between particle motion and interphase heat exchange also need more efforts.

\subsubsection{Forced convection}

Forced convection is usually encountered in gas turbines, combustors, fluidized beds, and other industrial processes. Here we mainly consider the following types of forced convection, namely the benchmark problems of uniform flow past single object with simple geometry, the heat transfer of flow past clusters, the investigations of heat transfer coefficient in a complex configuration, the turbulent heat transfer, and the heat transfer in fluidized bed systems.

Heat transfer of flow past single object with stationary or moving boundary has been extensively studied mainly as a benchmark problem for the validation of the thermal IB methods. A parametric summary of these studies is presented in Table 4. Most of these studies focus on low or moderate Reynolds numbers ranging from 10 to 200 with the direct-forcing method and the ghost-cell method, and all three types of thermal boundary conditions were considered. With the increase of the Reynolds number, the average Nusselt number is also increased due to a higher flow velocity and the resultant vortex shedding, thus leading to stronger interphase heat transfer. Considering the heat transfer rate among the three types of thermal boundary conditions, it is believed that the heat transfer coefficient is the highest for the Neumann-type thermal boundary condition (iso-heat-flux), while lowest for the Dirichlet (isothermal) boundary condition. The distributions of the local surface Nusselt number and the temperature of the heat transfer in the uniform flow past a single cylinder are shown in Figure 11. As can be seen that the surface temperature and Nusselt number in a Robin type case are smaller than those in a Neumann case (Luo et al. 2016b; Pan 2012). This is associated with the variation of surface temperature changing inversely with the local Nusselt number in a Neumann case (Zhang et al. 2008).

Heat transfer in clusters is much more complicated compared to that of flow past a single object. The particleaveraged Nusselt number could be increased or decreased due to particle-particle interaction. Tavassoli et al. (2013)

Table 4: A parametric summary of flow past single object with heat transfer using IB method.

\begin{tabular}{|c|c|c|c|c|c|c|}
\hline References & Method & $\begin{array}{r}\text { Grid } \\
\text { resolution }\end{array}$ & Geometry & Reynolds number & Object status & $\begin{array}{l}\text { Thermal } \\
\text { boundary }\end{array}$ \\
\hline Luo et al. (2016b) & Ghost cell & 70 & Circular cylinder & 20,40 & $\begin{array}{l}\text { Stationary, in-line } \\
\text { oscillating }\end{array}$ & $\begin{array}{l}\text { Dirichlet, Neu- } \\
\text { mann, Robin }\end{array}$ \\
\hline Xia et al. (2014) & Ghost cell & 32 & Circular cylinder & $50,100,200$ & Stationary & $\begin{array}{l}\text { Dirichlet, } \\
\text { Neumann }\end{array}$ \\
\hline $\begin{array}{l}\text { Santarelli et al. } \\
(2016)\end{array}$ & $\begin{array}{l}\text { Direct forcing } \\
\text { and ghost cell }\end{array}$ & 30 & Sphere & 10 & Stationary & $\begin{array}{l}\text { Dirichlet, } \\
\text { Neumann }\end{array}$ \\
\hline Zhang et al. (2008) & Direct forcing & 40 & Circular cylinder & $20,120,200,218$ & $\begin{array}{l}\text { Stationary, trans- } \\
\text { versely oscillating }\end{array}$ & $\begin{array}{l}\text { Dirichlet, } \\
\text { Neumann }\end{array}$ \\
\hline Paul et al. (2013) & Direct forcing & - & Elliptic cylinder & $50,100,150,200$ & Stationary & $\begin{array}{l}\text { Dirichlet, } \\
\text { Neumann }\end{array}$ \\
\hline Ren et al. (2013) & Direct forcing & 256 & Circular cylinder & $10,20,40,100$ & Stationary & Neumann \\
\hline Pan (2012) & Ghost cell & - & Circular cylinder & $20,40,100,200$ & Stationary & $\begin{array}{l}\text { Dirichlet, Neu- } \\
\text { mann, Robin }\end{array}$ \\
\hline $\begin{array}{l}\text { Pacheco-Vega } \\
\text { et al. (2007) }\end{array}$ & Direct forcing & - & $\begin{array}{l}\text { Circular cylinder, } \\
\text { sphere }\end{array}$ & $\begin{array}{r}40,80,120,150 \\
50,100,150 \\
200,220,300\end{array}$ & Stationary & Dirichlet \\
\hline $\begin{array}{l}\text { Suzuki et al. } \\
\text { (2018) }\end{array}$ & Direct forcing & 50 & Circular cylinder & $10,20,40$ & $\begin{array}{l}\text { Stationary, trans- } \\
\text { versely oscillating }\end{array}$ & $\begin{array}{l}\text { Dirichlet, } \\
\text { Neumann }\end{array}$ \\
\hline Wang et al. (2009) & Direct forcing & 96 & Circular cylinder & 20 & Stationary & Dirichlet \\
\hline $\begin{array}{l}\text { Dierich and Nikri- } \\
\text { tyuk (2013) }\end{array}$ & $\begin{array}{l}\text { The original IB } \\
\text { method }\end{array}$ & - & $\begin{array}{l}\text { Circular cylinder with } \\
\text { roughness }\end{array}$ & $\begin{array}{r}10,20,40,100, \\
200\end{array}$ & Stationary & Dirichlet \\
\hline Luo et al. (2017) & Ghost cell & $60^{*}$ & $\begin{array}{l}\text { Square cylinder, semi- } \\
\text { circular cylinder }\end{array}$ & $\begin{array}{r}100 \\
150\end{array}$ & Stationary & Dirichlet \\
\hline $\begin{array}{l}\text { Tavassoli et al. } \\
\text { (2013) }\end{array}$ & Direct forcing & 20 & Sphere & $\begin{array}{r}20,30,40,50,60 \\
100\end{array}$ & Stationary & Dirichlet \\
\hline
\end{tabular}

*Unit of diameter of semicircle or the edge of square. 
investigated a thermal flow of $T_{\infty}=275 \mathrm{~K}$ past 54 randomly packed stationary sphere particles with a constant temperature of $320 \mathrm{~K}$ by using the direct-forcing IB method. The Reynolds number was chosen as $R e=10,50$, and 100, and the solid fraction as $\phi=0.1,0.3$, and 0.5 . The small changes in the temperature and velocity fields in the vicinity of particles were well captured, as shown in Figure 12. It was found that great discrepancy existed in the local Nusselt number for every single particle, but a good agreement of averaged Nusselt number with Gunn's correlation (Gunn 1978) was obtained. Xia et al. (2014) studied the forced convection in a cluster of 20 randomly packed stationary sphere particles by using the high-order ghost-cell method. The solid fraction is $\phi=0.16$, and the Reynolds number is $R e=20,50$, and 100 . They reported that the flow patterns and drag force coefficients were like those in flow past a single sphere particle with an equivalent diameter. Nevertheless, the particle-averaged Nusselt number of the cluster was much lower than that of an isolated sphere particle under the same equivalent Reynolds number, showing that particle clusters severely lowered the heat transfer of each particle.

Many studies focus on the correlations of local and averaged Nusselt number in particle clusters with forced convection heat transfer in order to establish more accurate multiphase heat transfer models. With the multi-directforcing method, Wang et al. (2009) derived a correlation for the local Nusselt number distribution at each column of the staggered cylindrical tube bank. The temperature at the cylinder surface was kept constant and the Reynolds number was set as $R e=20$. The local Nusselt number distribution could be written as:

$$
N u(\theta, m)=N u(\theta, 2) \times 1.79^{2-m}, m \geq 2,
$$

where $N u(\theta, m)$ is the Nusselt number at the $\theta$ degree at the $m$-th cylinder's surface. In the work of Tenneti et al. (2013),

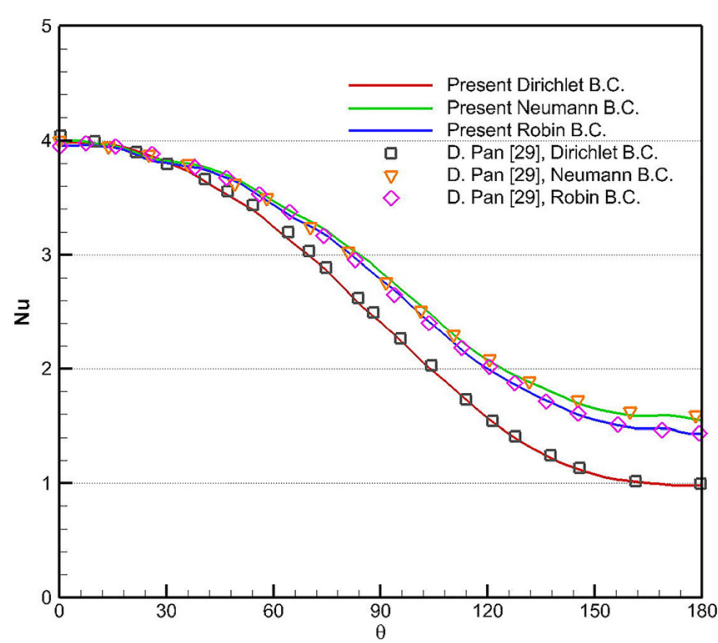

(A)

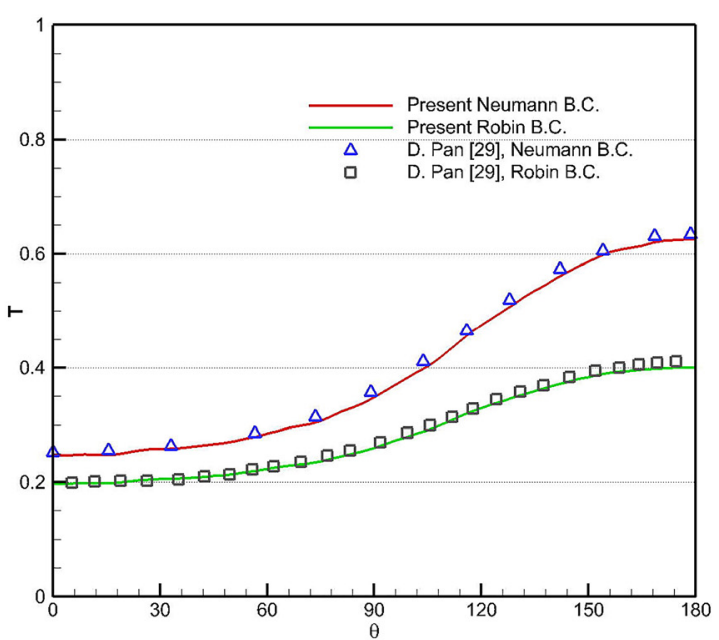

(B)

Figure 11: The local distribution of the Nusselt number (A) and temperature (B) at the surface of the cylinder particle. Reprinted from Luo et al. (2016b) with permission of Elsevier.

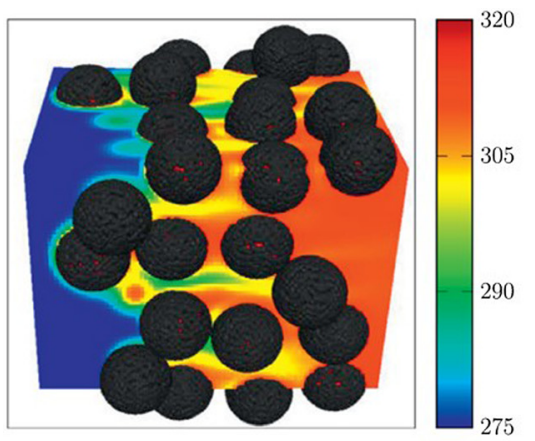

(A)

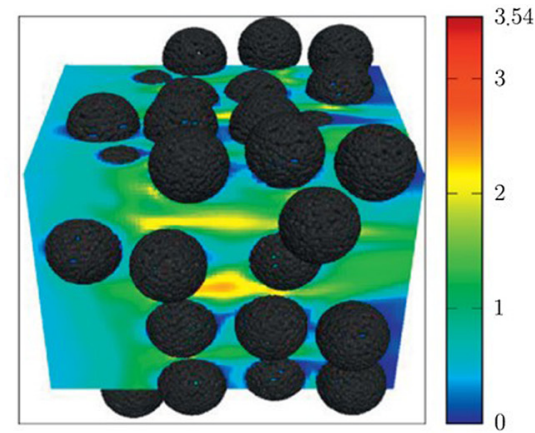

(B)
Figure 12: The temperature $(A)$ and velocity (B) distributions of fluid phase in a randomly packed cluster domain (54 particles) at $R e=50$ and $\phi=0.1$. Particles are represented by black spheres. Reprinted from Tavassoli et al. (2013) with permission of Elsevier. 
the heat transfer in a fixed cluster of sphere particles at constant temperature was studied by the PUReIBM. They concluded that for gas-solid systems with solid volume fraction $\phi<0.4$ and particle mean slip Reynolds number $R e_{m}>10$, the bulk fluid temperature would decay over a few particle diameters. However, in the case of $\phi>0.4$ and $R e_{m}<10$, particle heat transfer would significantly alter the bulk temperature. This result showed that the scale separation assumption in the modeling of the two-fluid method was not always valid. Later on, they studied the heat transfer in fixed assemblies of particles under the Dirichlet thermal boundary condition and proposed a new correlation of Nusselt number for volume fraction within 0.1-0.5 and particle slip Reynolds number within 1-100 (Sun et al. 2015). The newly proposed correlation was

$$
\begin{aligned}
N u= & \left(-0.46+1.77 \varepsilon+0.69 \varepsilon^{2}\right) / \varepsilon^{3} \\
& +\left(1.37-2.4 \varepsilon+1.2 \varepsilon^{2}\right) \operatorname{Re}^{0.7} \operatorname{Pr}^{1 / 3}
\end{aligned}
$$

where $\varepsilon=1-\phi$ is the porosity. Based on this correlation, they modified the heat transfer model for the two-fluid model. He and Tafti (2017) investigated the heat transfer in the flow past a fixed random assembly of spheres and ellipsoid particles at low to moderate Reynolds numbers under Neumann thermal boundary condition by using the ghost-cell method. The Reynolds number was varied from 10 to 200. The Prandtl number and solid volume fraction were set as 0.2 and 0.74 , respectively. The temperature variation at particle surfaces for both cases at $R e=200$ was clearly shown in Figure 13. It could be seen that the temperature at the particle surface could vary significantly with the changing of the azimuthal angle, and the particle position could alter the surface temperature. The average Nusselt number of spherical particles was in good comparison with existing data. In general, the mean Nusselt number was larger for ellipsoid particles than sphere particles, and particle orientation had a significant influence on the modulation of the flow and thermal fields around ellipsoid particles. A new correlation for the ellipsoidal particles of aspect ratio 1:2.5 was given as follows:

$$
\begin{aligned}
N u= & \left(1.49-0.885 \varepsilon+0.078 \varepsilon^{2}\right)\left(2.458-0.042 \operatorname{Re}^{1.09} \operatorname{Pr}^{1 / 3}\right) \\
& +\left(1.114-0.62 \varepsilon-0.08 \varepsilon^{2}\right) \operatorname{Re}^{0.7} \operatorname{Pr}^{1 / 3} .
\end{aligned}
$$

This correlation was suitable when the Reynolds number and solid fraction were in the range of $10 \leq R e \leq 50$ and $0<\phi \leq 0.35$, or of $50<R e \leq 200$ and $0.1 \leq \phi \leq 0.35$.

Most of the aforementioned studies focus on heat transfer of particles under stationary status at a relatively low Reynolds number without turbulence. However, the actual particle motion and heat transfer processes in a fluidized bed and other chemical and industrial equipment are usually free and strongly interacted with turbulence. Hence, the study of fully resolved particles interaction with turbulence is imperative.

With the high-order ghost-cell method, Xia et al. (2017) studied the heat transfer of a group of free-moving particles subjected to incoming isotropic turbulence with a characteristic Reynolds number of $R e=271.2$. The particle temperature was set as constant, and the solid volume fraction was set as 0.01 and 0.02 for two different cases. They found that the classical drag and Nusselt correlations for stationary particles, such as the Schiller-Naumann formula (Naumann and Schiller 1935) and Gunn's correlation (Gunn 1978), could hardly predict those for moving particles. In all the cases considered, the particle-averaged Nusselt number was higher than that predicted by Gunn's correlation (Gunn 1978). Compared to heavier particles, lighter particles were more pronouncedly affected by turbulent fluctuations so that the interphase heat exchange was much more significant, thus resulting in a higher average Nusselt number for lighter particles. Soon afterward, they carried out a fully resolved direct numerical simulation of spatially developing boundary layer laden with 1242 spherical particles at a characteristic momentum thickness

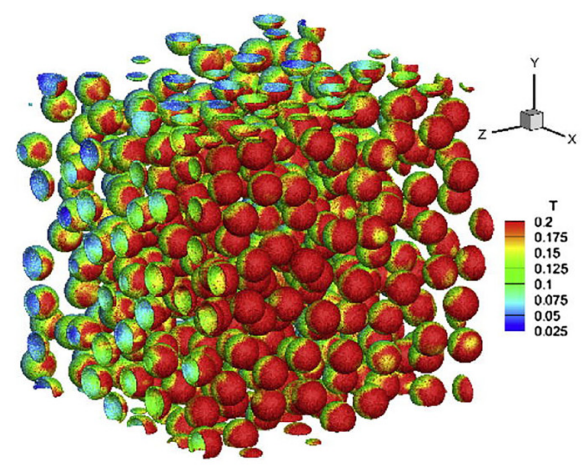

(A)

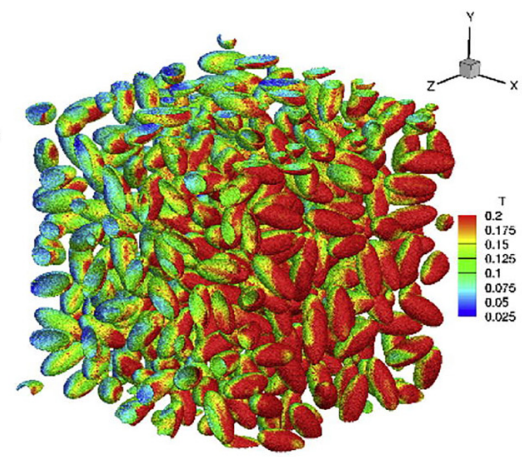

(B)
Figure 13: The dimensionless temperature variation at the surfaces of a fixed random assembly of spherical particles (A) and ellipsoidal particles (B) at solid fraction of $\phi=0.2$ and $R e=200$. Reprinted from $\mathrm{He}$ and Tafti (2017) with permission of Elsevier. 


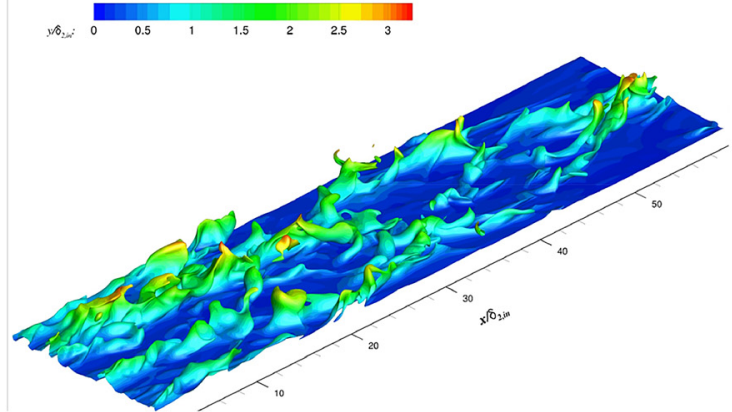

(A)

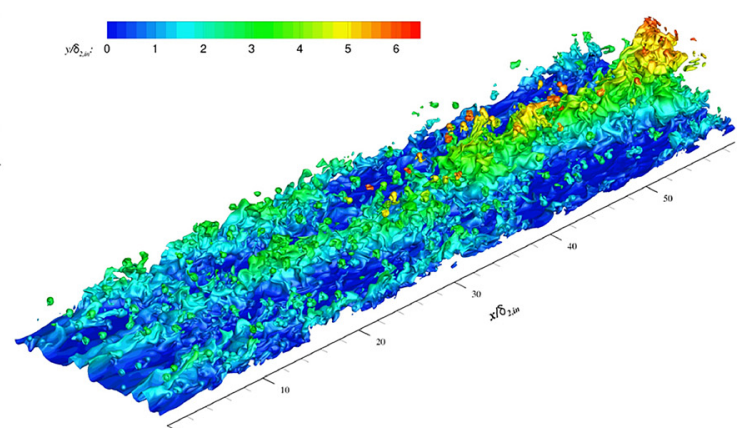

(B)

Figure 14: Isosurfaces of the dimensionless temperature $T^{\star}=0.5$ in the turbulent thermal boundary layer for the no particle case (A) and multiphase case (B), colored by wall-normal height. Reprinted from Xia et al. (2018) with the permission of Elsevier.

Reynolds number $R e_{\delta_{2}}=800$ by using the high-order ghost-cell IB method (Xia et al. 2018). The solid volume fraction was very low at $\phi=0.25 \%$. It was pointed out that finite-size particles were strong thermal and kinetic energy sources to the turbulent boundary layer, thus leading to the increase in velocity fluctuations and wall-normal turbulent heat flux. However, since particle surface temperature was kept the same as the wall temperature, the existence of particles in the near-wall region damped temperature fluctuation. This could also be demonstrated in the isosurfaces of dimensionless temperature of 0.5 , as shown in Figure 14. Compared with the no particle case in Figure 14A, the isosurfaces in Figure 14B are thicker and the temperature near the wall is more uniform due to the existence of particles so that temperature fluctuations in the near-wall regions are suppressed.

Although the computational cost is very high, many studies directly focus on the heat transfer in fluidized bed systems aiming at revealing the complex multiscale heat transfer process by using the IB methods. Feng and Musong (2014) studied the heat transfer of 225 heat spheres in a fluidized bed using the direct-forcing method. Five cases with fluidization velocity varying from $0.035 \mathrm{~m} / \mathrm{s}$ to $0.055 \mathrm{~m} / \mathrm{s}$ were studied with a particle diameter of $d=0.00635 \mathrm{~m}$. Characteristic hydrodynamic and thermal properties showed reasonable agreements with other literatures. It was found that the particle-averaged Nusselt number increased with the enhancement of the fluidization velocity and the bed height. This method was further extended to study the fluidization in a narrow channel with 1024 particles (Feng and Roig 2014). Fluidization velocity was set as $0.04 \mathrm{~m} / \mathrm{s}$ and $0.045 \mathrm{~m} / \mathrm{s}$ with the same particle diameter as that in the work of Feng and Musong (2014), and similar results were obtained. Deen et al. (2012) studied a pseudo-two-dimensional fluidized bed system with 1296 sphere particles with a diameter of $d_{p}=0.005 \mathrm{~m}$ by using the ghost-cell method. The minimum fluidization velocity was $1.17 \mathrm{~cm} / \mathrm{s}$, the terminal velocity was $39.6 \mathrm{~cm} / \mathrm{s}$, and the superficial liquid velocity was $8 \mathrm{~cm} / \mathrm{s}$. The flow field was highly uniform under the influence of particles, and significant bed expansion occurred due to the high superficial velocity, as shown in Figure 15. This high superficial velocity also led to the great spatial variation of fluid-particle heat transfer coefficients.

In summary, the IB methods, particularly the directforcing method and the ghost-cell method, have been extensively used in fully resolved simulations of multiphase flows with forced convection. The particles are
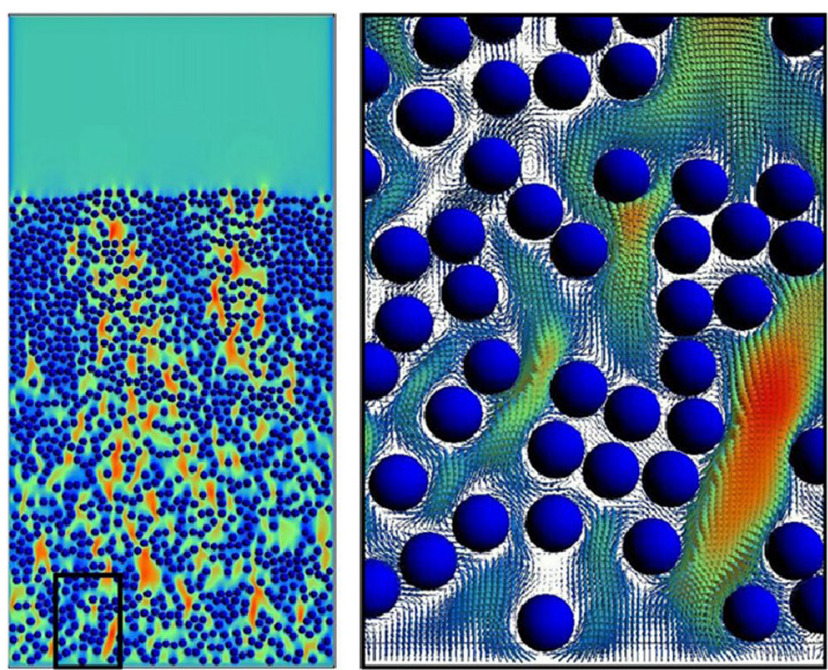

Figure 15: The particle configuration in a subdomain of the fluidized bed (left) and the velocity profile of the fluidized agent in a partial enlarged view (right); blue spheres represent the fluidized particles while red and blue represent the higher and lower velocities, respectively. Reprinted from Deen et al. (2012) with the permission of Elsevier. 
generally assumed to be stationary in the study of particle clusters with heat transfer, and it has been demonstrated that the particle motion would significantly alter the heat transfer process as well as the Nusselt number (Xia et al. 2017). In addition, the distribution of local Nusselt number varies a lot in different locations at the particle surface, and the rotation of particles should be considered in the future work in deriving a general correlation for Nusselt number. The interaction of fully resolved particles and heat transfer in turbulent flow is also far from clear, and more investigations on particle-turbulence-heat transfer interactions should be carried out. Furthermore, most of the aforementioned studies are limited to small-scale system loaded with few particles. Fully resolved numerical simulations of multiphase heat transfer in a large-scale system laden with thousands of particles are on the way ahead.

\subsubsection{Conjugate heat transfer}

In the aforementioned studies, the effects of heat conduction within the solid were generally neglected. However, in realistic industrial applications, especially when the thermal conductivity of the solid phase is smaller than that of flow, considering conjugate heat transfer at the interphase surface would be more physically necessary.

Because of the coupling of thermal boundary conditions at both sides of the boundary surface in conjugate heat transfer, numerically modeling these coupled equations is the first step in the IB methods. Kang et al. (2009) transferred the CHT boundary condition to the Dirichlet boundary condition of temperature at the fluid side and Neumann boundary condition of heat flux at the solid side. The temperature and flux at each target boundary are interpolated from the opposite side, respectively. With a similar idea, Crocker et al. (2014) interpolated the heat flux through the one-side difference in each side of the interface under a cut-cell method. Under the framework of the ghostcell method, Nagendra et al. (2014) and Das et al. (2016) applied the ghost cells at both fluid and solid regions to satisfy the thermal balance. Recently, a simplified numerical algorithm was proposed to solve the conjugate heat transfer problem. By assuming that the products of density and heat capacity $\rho C_{p}$ are identical in both fluid and particle phases, the original governing equation of energy, i.e., $\rho C_{p}\left(\frac{\partial T}{\partial t}+u \cdot \nabla T\right)=\nabla \cdot(\lambda \nabla T)$, could be reduced to $\frac{\partial T}{\partial t}+$ $u \cdot \nabla T=\nabla \cdot(\alpha \nabla T)$, where $\alpha=\lambda /\left(\rho C_{p}\right)$ is the thermal diffusivity. This simplified equation could be solved in the whole computational domain including the particle region. Consequently, the calculation of the heat flux at the interphase surface is the only remaining question.
Ardekani et al. (2018a) used the volume of fluid method to calculate the velocity and thermal diffusivity at the interphase surface. In the work of Sato et al. (2016), the heat flux at the interfacial boundary was decomposed into the normal and tangential component, so that one more constraint of $\partial T /\left.\partial \bar{\tau}\right|_{\text {fluid }}=\partial T /\left.\partial \bar{\tau}\right|_{\text {solid }}$ was applied.

The first attempt to employ the IB method to deal with the CHT problem was made by Iaccarino and Moreau (2005). They combined the ghost-cell method with a RANS solver and local grid refinement technique to investigate the CHT problem of an automotive engine cooling fan system under natural and forced convective conditions. Their results agreed well with those obtained under the body-conformal grid system, which showed the capability of the IB method to study CHT problems. Kang et al. (2009) studied CHT problems of turbulent flows on a heated cylinder with the ghost-cell method. They found that the inclusion of solid conduction could lead to better results of local Nusselt numbers compared with experiments.

Takeuchi et al. (2013) extended the direct-forcing method of Kajishima et al. (2001) to include heat conduction to study the natural convection of a group of movable two-dimensional cylinders in a square enclosure with a cold bottom and a hot top considering the $\mathrm{CHT}$ at the cylinder surfaces. The Prandtl number, particle-fluid density ratio, and specific heat ratio are all set as unity. When the heat conductivity ratio is low, such as $\lambda_{s} /$ $\lambda_{f}=10^{-3}$ and $10^{\circ}$, the fluid and particles were found to circulate the domain center in one direction. However, at a high heat conductivity ratio, i.e., $\lambda_{s} / \lambda_{f}=10^{1}$ and $10^{2}$, particles tended to oscillate around the domain center at a frequency relevant to the thermal diffusion time scale. They further investigated the effects of solid conductivity to heat transfer (Tsutsumi et al. 2014) and found that in the case of particles with lower solid conductivity, such as $\lambda_{s} /$ $\lambda_{f}=10^{-3}$, particles tended to accumulate near the wall region; while in the case of higher solid conductivity, such as $\lambda_{s} / \lambda_{f}=10^{3}$, particles distributed more uniformly. Particles near the wall region would lower the heat transfer from hot wall to fluid, thus leading to a decreased Nusselt number. Later on, this method was extended to a threedimensional case considering the effects of particle contacting heat transfer (Takeuchi et al. 2015). They found that when particles formed a chain contacting structure, a burst of heat flux occurred in the region across several particle diameters, as highlighted in Figure 16. This burst would cause a large temperature gradient near the particle chain and efficiently enhance fluid heat transfer and interphase heat exchange. 
Das et al. (2017) applied the ghost-cell IB method to simulate the heat transfer of a randomly packed structure of spherical particles in slender fixed-bed reactors. A CHT boundary condition was imposed on the surface of the spherical particles. The particle diameter was set as constant, while the diameter of the cylindrical domain was varied from 4 to 12 times of particle radius. Particle Reynolds number was varied from 1 to 500 . It was found that increasing the solid conductivity from 1 to 100 would slightly enhance the overall heat transfer coefficient. This enhancement was further flattened at a high particle Reynolds number of $R e=500$, which indicated that conjugate heat transfer had little effect on the overall wall-to-bed heat transfer. Further, this method was extended to simulate flow through a three-dimensional highly complex random solid foam structure under the CHT boundary condition (Das et al. 2018). The conductivity ratio was chosen as $\lambda_{s} /$ $\lambda_{f}=10,100$, and 1000. Although the flow field was little influenced by the conjugate heat transfer process, a higher temperature distortion could still be observed at $\lambda_{s}$ / $\lambda_{f}=1000$, as shown in Figure 17.
Ardekani et al. (2018a) combined the direct-forcing method and the volume of fluid method to study the conjugate heat transfer of the rigid spherical particle in a laminar Couette flow. The interphase heat transfer was studied by varying the particle Reynolds number from 1 to 16 , the volume fraction of particles from 3 to $30 \%$, and ratio of the thermal diffusivity of the particle to the fluid from 0.1 to 1 . They found that when the particle volume fraction is lower than $10 \%$, the increase of the particle Reynolds number could effectively enhance the interphase heat transfer. However, at higher volume fractions, the increase of Reynolds number cannot prompt the overall interphase heat transfer due to the larger enhancement of viscosity effect. It was also pointed out that the contribution of heat conduction in solid phase to the overall heat transfer was reduced as the particle Reynolds number increased. Using the same method, Ardekani et al. (2018b) studied the motion and heat transfer of spherical particles in a pipe flow at laminar and turbulent regions with $R e=370$ and 5300, respectively. The solid volume fraction was varied from 0 to $40 \%$. In the laminar regime, fully resolved particles greatly enhanced
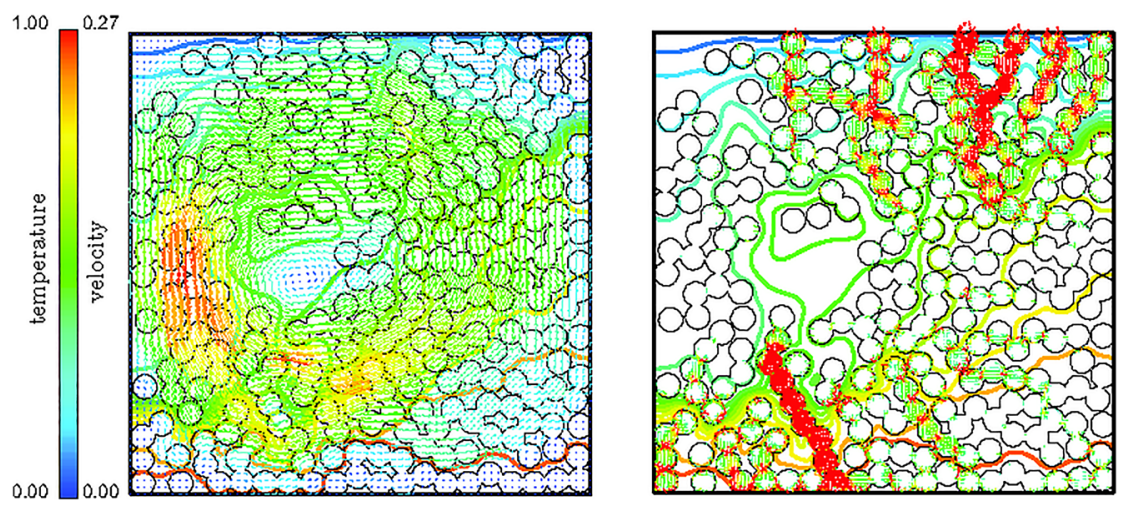

Figure 16: Instantaneous flow field (left) and conductive heat flux (right) of a 2D natural convection problem with multiple particles in a square domain. The vectors represent fluid velocity and conductive heat flux, respectively, while in both figures the isocontours represent the temperature. Particles are represented by black circles. Reprinted from Takeuchi et al. (2015) with permission of Begell House.

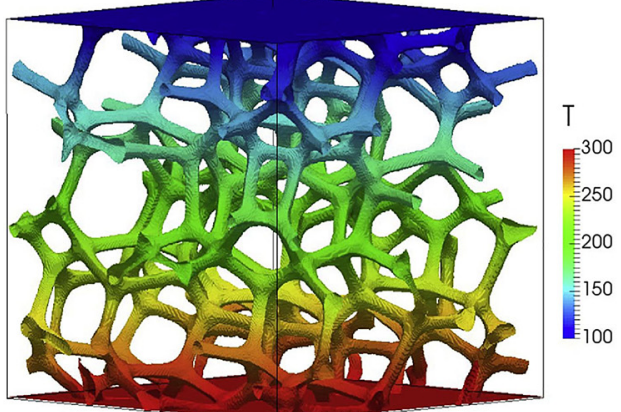

(A)
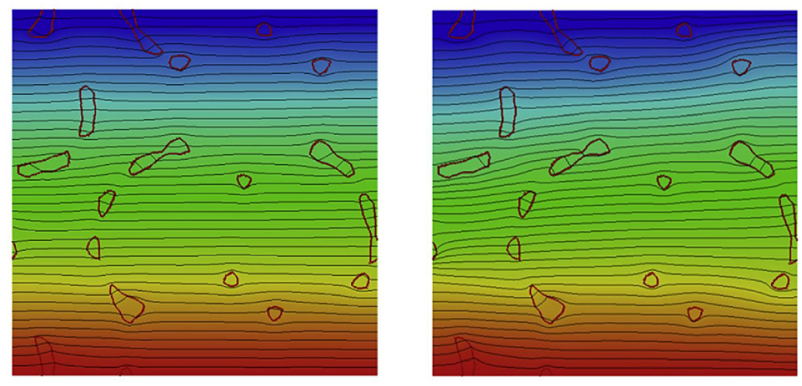

(B)

Figure 17: (A) The temperature at the solid surface of random foam structure at $\lambda_{s} / \lambda_{f}=1000$. (B) Temperature contours at mid-plane for $\lambda_{s} /$ $\lambda_{f}=10$ (left) and $\lambda_{s} / \lambda_{f}=1000$ (right). The irregular hollow structures are crossed section of random foam. Reprinted from Das et al. (2018) with permission of Elsevier. 
heat transfer (with a maximum of 330\%) and wall drag compared with the case of no particle. However, in the turbulent regime, particles would relaminarize the turbulent pipe flow and severely damp the heat transfer. Only in the case of a volume fraction of $5 \%$, the heat transfer coefficient was higher than that in the single-phase turbulent flow.

Apart from application in multiphase problems, CHT also gains popularity in characterizing the complex thermal boundary conditions of a wall or confined geometry. One noteworthy application is considering CHT in investigating flame-wall interaction (FWI) problems (Kedia et al. 2014; Michaels and Ghoniem 2016). Using the IB method to study CHT and FWI problems is quite straightforward. Consider the FWI problem, only the no-penetration boundary condition of species should be additionally satisfied at the immersed interface as $\partial Y /\left.\partial \bar{n}\right|_{\Gamma}=0$, and this Neumann boundary condition is easy to implement in the framework of the IB methods (Kedia et al. 2014).

To sum up, significant efforts have been made to apply the IB methods to simulations of the CHT problems. Numerical results demonstrate that including the conduction heat transfer within the solid could reproduce better collapse on experimental results. However, the multiphase heat transfer model considering the effect of CHT is rarely investigated. Due to the complexity in characterizing CHT problems, the influence of CHT on fluid convective heat transfer, interphase momentum, and energy transfer needs further investigations.

\subsection{Mass transfer problem}

The multiphase flow with mass transfer is frequently encountered in solid/liquid fuel combustion, permeation at the porous surface, and so on. Although there have been many modeling studies toward multiphase combustion and reaction system with traditional methods, a systematic fully resolved study is still in need to uncover the underlying physics of multiphase mass transfer. In recent years, lots of efforts have been made to investigate mass/ species transfer problems since the capability of the IB methods on handling species transport problems has been demonstrated by Huang et al. (2009) with a simulation of mass diffusion across a fixed permeable interface.

The application of the IB methods in mass transfer problems mainly contains two types, namely the species permeation and heterogeneous reaction. Modeling of mass transfer boundary conditions is based on the reactions or permeation at the immersed boundary. In the species permeation or diffusion-convection problems, the mass transfer process is governed by species diffusion and convection. While for the problems involving heterogeneous chemical reactions, the mass transfer on the surface contains diffusion, reaction, and other transport phenomena such as Stefan flow (Kee et al. 2005). Furthermore, the species transport equation is usually coupled with the energy equation, which leads to fully coupled momentumspecies-energy problems.

\subsubsection{Permeation problems}

The permeation problems are widely encountered in the studies of medical science, such as blood cells or medicine delivery. Gong et al. (2014) extended the idea of Huang et al. (2009) to the multiple moving interface problems governed by the convection-reaction-diffusion equation by using the original IB method. The effects of mechanical and geometry properties were analyzed. Figure 18 shows the oxygen transfer over the IB. Simulations indicated that the effect of the hematocrit value became a constant when the value was above 0.362 . The stiffness influenced the oxygen transfer rate of the symmetrically distributed cells significantly, while it showed little effect on the asymmetric configuration. Wei et al. (2014) presented a directforcing IB method for simulations of sorption behaviors of

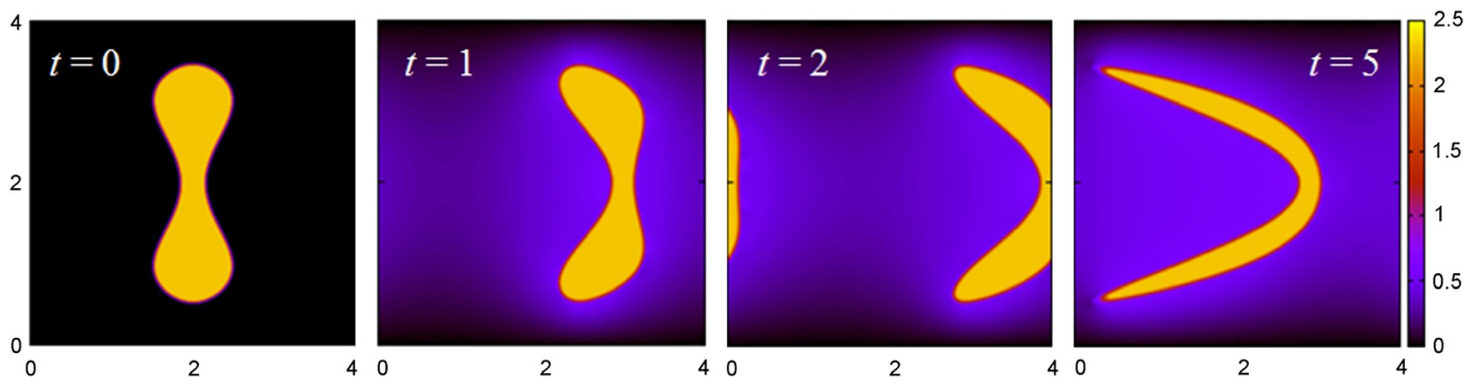

Figure 18: Contours of the dimensionless concentration for oxygen transfer over the passively moving and deforming interface immersed in a steady flow field at different time instants. The yellow region represents the interior of the interface. Reprinted from Gong et al. (2014) with permission of Elsevier. 
porous media. A sink term was added in the species transport equation to represent the porous effect of the smaller-scale microporous material. It was found that the species could not be absorbed by a single layer of the porous structure, which could be used to determine the number of microscopic porous layers and provide valuable information for the design of the gas filter. Roghair et al. (2016) studied heat and mass transfer in a bubble swarm by combining the original IB method with the FT method under the framework of a finite-volume solver. The method was suitable to simulate the mass transfer of bubble swarms at a gas holdup range of $4-40 \%$. Results showed that the mass transfer rate to the liquid phase just increased slightly with the increase of the gas holdup.

The key to the permeation problem is how to enforce the jump conditions of the concentration and pressure on the surface. Jayathilake et al. (2010) studied the effect of deformation and swelling of the membrane on the permeability properties by using the IIM to implement the jump conditions of solute concentration at the boundary surface. The jump conditions were calculated based on the Kedem-Katchalsky relations (Kedem and Katchalsky 1958). The accuracy of this method was validated with numerical errors within $1 \%$. The simulations showed that the osmotic load influenced the capsule swelling and deformation observably. The capsule took a longer time to reach an equilibrium state when the osmotic load was smaller. Besides, the initial solute concentration ratio had a positive effect on the capsule swelling. This method was also applied by Jayathilake et al. (2011) to simulate the adhesive capsule, where the capsule-substrate adhesion was enforced using an adhesion potential (Seifert 1991). The solute absorbed by the vessel can be enhanced by increasing the diffusive permeability of the membrane. The capsule moving along the vessel increased the solute absorption by the vessel, and the adhesion strength influenced the solute transfer positively. Figure 19 shows the deformation and movement of the capsule. The solute concentration was much larger in a nonadhesive capsule, while the concentration inside an adhesive capsule was little different from that outside. The main reason was that adhesive capsules tended to move toward the wall, and more solute mass would be absorbed.

Musielak et al. (2009) studied the nutrient acquisition of diatom chains in different kinds of background flows and nutrient sources by using the original IB method. The uptake of nutrients was transmitted to the fluid domain by using a reaction term in the species concentration equation. The role of the mechanical properties of the chain between the diatoms was clarified, and some noteworthy points about the evolution of diatoms were pointed out. For the chain with four or five cells, the stiff chains took in more nutrients than solitary cells, and this effect was enhanced when the stiffness increased. Lambert et al. (2013) studied the active suspension of particles in a thin film by using the direct-forcing IB method. The nutrient uptake and swimming accumulation tendency of particles were investigated. A detailed analysis of the effect of the absorption rate, the swimming speed, and the volume fraction on nutrient uptake was performed. They clarified that although swimming took energy, it was still a winning strategy for microorganism to survive in the relatively dense suspensions.

\subsubsection{Mass transfer with heterogeneous reactions}

Researches involving heterogeneous reactions are very complicated since the heat and mass transfers are fully coupled, and the boundary conditions might be influenced by the reactions. The early studies of mass transfer with heterogeneous reactions were simplified by ignoring the thermal properties and complicated transport phenomena (e.g., Stefan flow) of the heterogeneous reactions. The coupling effects of these multiphysics were only investigated in the recent years.

In simplified studies, the Stefan flow phenomenon and energy equation, as well as the chemical reaction heat, are sometimes ignored in imposing boundary conditions. Yao and Fogelson (2012) formulated the direct-forcing IB method as an augmented system to simulate the chemical transport in the fluid field outside reactive particles. The forcing term and the value of species concentration were solved simultaneously in an augmented system of equations, which consisted of discrete chemical transport equations and discrete boundary conditions so that the boundary conditions were enforced explicitly. It was demonstrated to be more stable and accurate than the traditional direct-forcing IB method, which calculated the correcting forcing term using a prediction species concentration value of the next time step (the so-called predictor-corrector forcing method). The Stefan flow was neglected, and the energy equation was not included. Sulaiman et al. (2019) used the IB method coupling with the fictitious domain method to simulate the convective mass transfer around reactive particles in an incompressible flow. The ranges of Reynolds number and Schmidt number were from 10 to 100 and 0.5 to 10 , respectively. The species diffusion and reactions inside the particle were considered. The chemical reaction was assumed to be first order and had no backward influence on the flow field. The convective heat transfer and interphase heat transfer, as well as the Stefan flow, were neglected. The effect of the reaction 

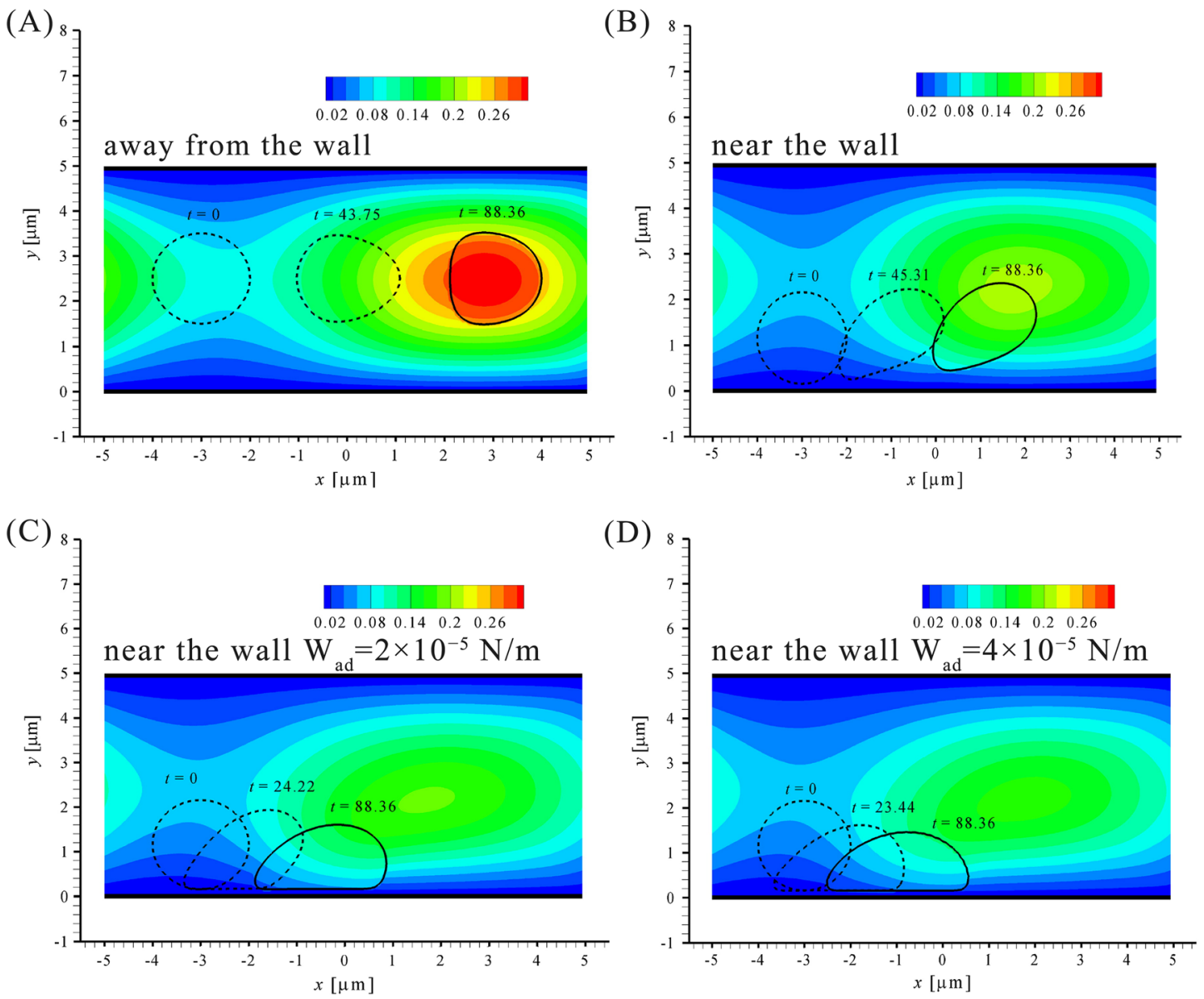

Figure 19: The deformation and movement of nonadhesive $((A)$ and $(B))$ and adhesive capsules with different adhesive coefficients $W_{a d}((C)$ and (D)); the contours represent the solute concentration at the time of $t=88.36 \mathrm{~s}$. The dashed $/$ solid circles represent the capsules at different time instants. Reprinted from Jayathilake et al. (2011) with permission of Elsevier.

was treated as a conjugate mass transfer problem due to the continuity of species concentration and species normal flux. Figure 20 shows the isosurfaces of concentration within and outside three aligned reactive spheres, which are significantly dependent on the Damköhler number. Accordingly, a modified correlation of Sherwood number for a particle with an internal first-order reaction was proposed, which was based on an existing nonreactive Sherwood number correlation.

In the studies including heat transfer effect, the coupling of energy equation and heterogeneous reaction at the immersed surface poses more difficulties in constructing the coupled boundary conditions. Deen and Kuipers (2013, 2014a, 2014b) extended the direct-forcing method to simulate coupled heat and mass transfer in reactive dense particles-laden flows. The Reynolds numbers of the exothermal chemical reactive particles were from 120 to 240 (Deen and Kuipers 2014b), and the mass transfer was simplified as a completely mass- transfer-limited condition. Several cases were presented under forced convection accompanied by a fast exothermic surface reaction. It was found that the mass transfer coefficients agreed well with the empirical equation (Deen and Kuipers 2013) and the correlation proposed by Gunn (Gunn 1978) for the cases of isolated particles and dense arrays, respectively. However, the mass transfer of a single particle in the dense array had a remarkable variation due to the preferred pathways of the fluid (Deen and Kuipers 2014b). Thereafter, a ghost-cell IB method was developed to simulate the mass transfer in the dense fluid-particle system (Lu et al. 2018a). The Reynolds numbers varied from 20 to 400, and the Damköhler numbers varied from 0.001 to 1000 . The first-order surface reaction was considered instead of an infinitely fast reaction in the previous study. However, the Stefan flow was still neglected. As a result, the species boundary condition has the form of the Robin boundary condition. Figure 21 shows the concentration field of the dense particle system at different Damköhler 


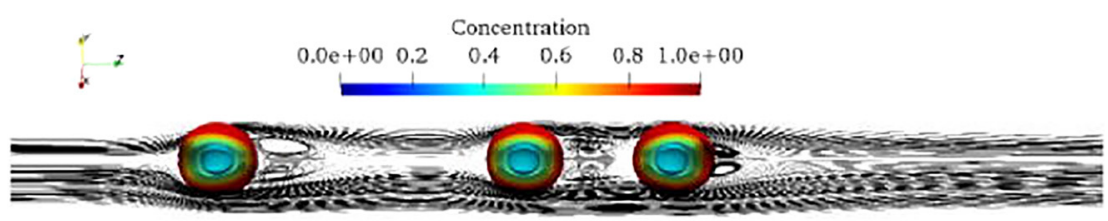

(A) $\phi^{2}=40$

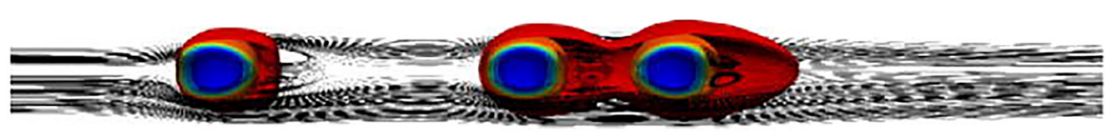

(B) $\phi^{2}=200$

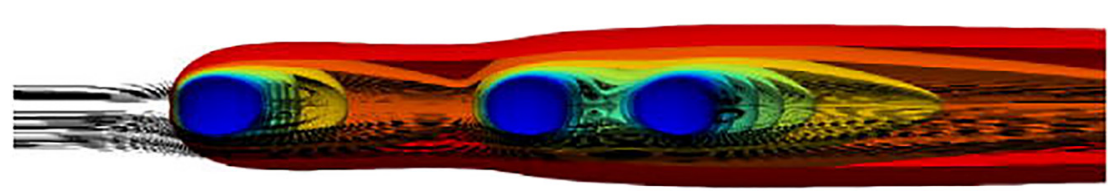

(C) $\phi^{2}=\infty$
Figure 20: Steady-state convectiondiffusion process in the flow past three aligned reactive spheres at different Damköhler numbers $\phi^{2}$; isosurfaces represent the species concentration. Reprinted from Sulaiman et al. (2019) with permission of Elsevier. numbers. With the increasing of Damköhler numbers, the reactant decayed quickly in the front part of the array, and full conversion of the reactant was achieved. The Sherwood number was influenced by the Damköhler number in the particle array. The temperature and species concentration profile of the dense particle array were compared with the results calculated according to the 1D heterogeneous model ( $\mathrm{Lu}$ et al. 2018c), and good qualitative agreement was achieved. Nevertheless, the discrepancy caused by the heterogeneity of the array was amplified in the conditions of a high Reynolds number. The same method was also utilized to study the mass transfer in a catalyst cluster when diluted by inert particles ( $\mathrm{Lu}$ et al. 2018b). The distribution of two kinds of particles led to a large variation of the mass transfer behavior. When the catalyst particle was surrounded by inert particles, the mass transfer became more active, especially at small cluster proximities.

Nevertheless, the aforementioned studies do not consider the Stefan flow effect. Inclusion of Stefan flow and heat transfer caused by heterogeneous reactions would be much more physically realistic. McGurn et al. (2013) investigated the conjugate heat transfer and mass transfer processes associated with charring solids. The moving interface was described by a level-set method, and the ghost-cell method was used to enforce the boundary conditions. The effects of Stefan flow were superimposed through an explicit source term deposition into the Eulerian flow field. Lupo et al. (2019) presented a new direct-forcing IB method based on Breugem's study (2012), which can fully capture the momentum, energy, and mass exchanges at the interface. Several validation cases of evaporating droplets were performed, including fixed rate and free evaporation of a static droplet, displacement of a droplet by Stefan flow, and evaporation of a hydrocarbon droplet in homogeneous isotropic turbulence. The Stefan flow velocity, temperature, and species distribution showed good agreements with analytical solutions and experimental data for both laminar and turbulent flows. Luo et al. (2016b) improved a ghost-cell IB method so that it could handle all the Dirichlet, Neumann, and Robin types of boundary conditions and further extended it to perform a fully resolved simulation of char particle combustion (Luo et al. 2018). In this study, the surface species transfer was a balance of diffusion, Stefan flow, and the heterogeneous reaction. The velocity of the interface was under a combined effect of the Stefan flow and the particle shrinking velocity. The heat transfer on the surface was coupled with a heterogeneous reaction. The char surface temperature was kept constant and varied from 1500 to $1900 \mathrm{~K}$ in different cases, and the Reynolds numbers were in a range of 2.5-30.0. The method was successfully validated against the experiment (Makino et al. 2003), and the jump of the combustion rate due to the changing of the dominant reaction can be captured accurately. Figure 22 shows the flame shape around the char particle under different conditions. It can be seen that the CO flame was detached from the surface when the particle temperature was high enough. The gas-phase reaction in the boundary was always transport-limited, and the drag force coefficient and the Nusselt number became larger due to the chemical reactions. Furthermore, this method was extended by Zhang et al. (2020) to investigate the effects of heterogeneous reactions and homogeneous reactions on 


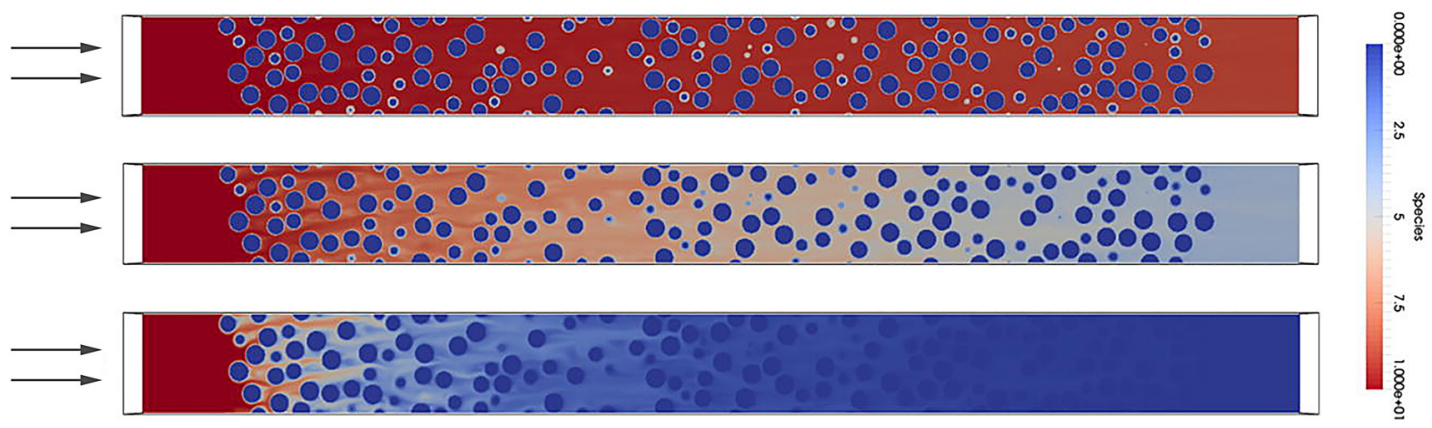

Figure 21: Concentration contours in the central slice of flow past the fixed particle array at three Damköhler numbers $(0.1,1,10$ from top to bottom). Reprinted from Lu et al. (2018a) with permission of Elsevier.

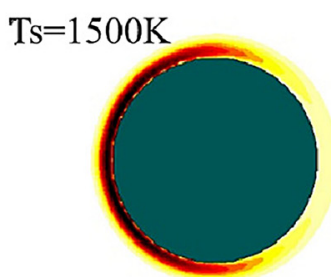

$\mathrm{Ts}=1750 \mathrm{~K}$

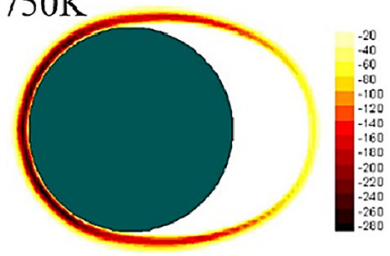

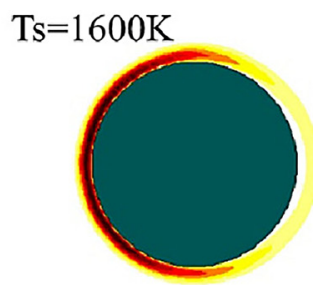

$\mathrm{Ts}=1800 \mathrm{~K}$

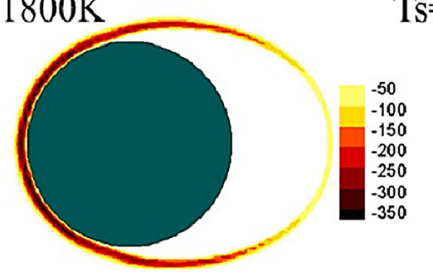

$\mathrm{Ts}=1700 \mathrm{~K}$

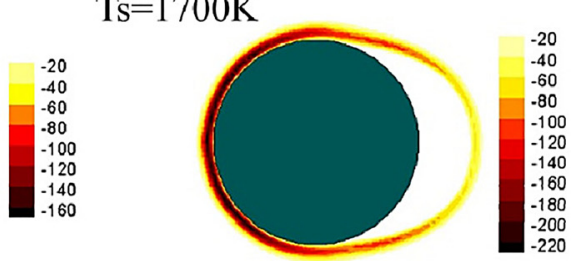

$\mathrm{Ts}=1900 \mathrm{~K}$

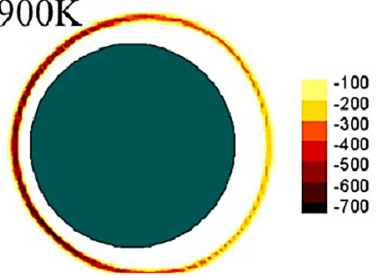

Figure 22: CO flame (shown as the contour of $\mathrm{CO}$ consumption rate) around the burning char particle (green cylinder in the figure) at different particle temperatures. Reprinted from Luo et al. (2018) with permission of John Wiley and Sons.

the drag force of a char particle. Simulation results indicated that both heterogeneous and homogeneous reactions tended to increase the pressure drop along the particle because of the accumulation of reactants at the rear region of the particle. Meanwhile, the high particle temperature led to high viscosity of gas phase in the particle boundary layer, which further increased the drag force.

In summary, the study of multiphase flow with mass transfer and the heterogeneous reaction has been carried out by many researchers, and a lot of achievements have been obtained. However, how to model the boundary condition physically as much as possible remains an open question. So far, there is no boundary model that considers the effect of finite reaction rate, the Stefan flow, and heat and mass transfer within in the particle at the same time. Although sophisticated, developing a realistic boundary model for the heterogeneous surface reaction is in need. Moreover, improved correlations for multiphase transport phenomena can be obtained with the help of the IB methods, which will further improve the predictive capability of traditional coarse models for industrial applications.

\section{Conclusions and future challenges}

With the development of large-scale parallel computing and numerical algorithms, the IB method has emerged as a powerful tool for fully resolved simulations of multiphase transport phenomena in the recent decades. Methodology development of two major types of IB methods and relevant applications to fully resolved simulations of multiphase isothermal flows, heat, and mass transfer problems have been reviewed in this article. From the perspective of methodology, we classify the IB method into two types, namely the artificial boundary method and the authentic boundary method, based on whether the interphase coupling conditions are numerically 
imposed on an artificially created region or only on the actual boundary. The artificial boundary method is easier to implement, but it suffers from inaccurate description of the boundary surface and worse conservation. Although the authentic boundary method could better represent the actual boundary, the difficulty arises in the construction of high-order numerical schemes, especially for moving and complex geometries. In the applications of the IB methods to multiphase transport, the focus is placed on natural convection within an enclosure, thermal characteristics of multiphase flow, heat correlations, CHT problem, permeation problem, and heterogeneous reaction. New findings have been obtained to better understand the underlying physics and develop new models.

Despite all these achievements, some open issues still exist in the field. The first issue is about the strict representation of the IB. Without body-conformal grid systems, special treatments, such as combining with the cut-cell method (Seo and Mittal 2011b), dynamically correcting the interface locations (Li et al. 2012), or constructing additional kinematic constraint imposed by the IB to the flow (Spizzichino et al. 2019a), are required to achieve better representation of the boundary surface as well as conservation property for the artificial boundary method, which gives rise to additional mathematical and numerical difficulties. This problem is quite disturbing, especially in simulations of high Reynolds number flows. Inaccuracy in the vicinity of the embedded body would lead to numerical instability, even divergence. Secondly, fully resolved simulations of realistic three-dimensional particle-laden dense flows need too many computational resources compared with the traditional Eulerian-Eulerian and Eulerian-Lagrangian methods. Using advanced grid generation techniques could partially alleviate this problem. However, the largest-scale laboratory fluidized bed simulation could only fully resolve around $O\left(10^{4}\right)$ particles so far, which is far from realistic industrial applications. Thirdly, there is a lack of comprehensive understanding of the modeling of boundary condition for mass transfer with heterogeneous surface reactions. The modeling of this boundary condition is quite sophisticated due to the strong and complicated coupling of momentum-energy-mass at the surface. As a result, development of the IB method in this domain is scarce, and many assumptions have been introduced to simplify the boundary conditions.

To overcome these issues, more realistic and robust descriptions of interphase coupling conditions should be developed in the future, especially for multiphase flows with heterogeneous reactions and surface mass transfer. In addition, advanced and efficient numerical techniques such as the adaptive mesh refinement and the accelerating algorithms are favorable to be incorporated into the IB methods to reduce computational cost. Finally, developing advanced subgrid models for interphase momentum, heat, and mass transfer that are usually used in the framework of RANS or LES based on the database of fully resolved simulations is another important direction in the field, which will essentially benefit industrial applications.

Author contribution: All the authors have accepted responsibility for the entire content of this submitted manuscript and approved submission.

Research funding: This study was supported by the National Science Fund for Distinguished Young Scholars (51925603) and the National Key Research and Development Program of China (2017YFB0601805). The authors are grateful for that.

Conflict of interest statement: The authors declare no conflicts of interest regarding this article.

\section{References}

Alan Wei, Z., Charlie Zheng, Z., and Yang, X. (2014). Computation of flow through a three-dimensional periodic array of porous structures by a parallel immersed-boundary method. J. Fluid Eng. 136: 040905.

Angot, P., Bruneau, C.-H., and Fabrie, P. (1999). A penalization method to take into account obstacles in incompressible viscous flows. Numer. Math. 81: 497-520.

Ardekani, M.N., Abouali, O., Picano, F., and Brandt, L. (2018a). Heat transfer in laminar Couette flow laden with rigid spherical particles. J. Fluid Mech. 834: 308-334.

Ardekani, M.N., Asmar, L.A., Picano, F., and Brandt, L. (2018b). Numerical study of heat transfer in laminar and turbulent pipe flow with finite-size spherical particles. Int. J. Heat Fluid Flow 71: 189-199.

Balachandar, S. and Eaton, J.K. (2010). Turbulent dispersed multiphase flow. Annu. Rev. Fluid Mech. 42: 111-133.

Bao, Y., Kaye, J., and Peskin, C.S. (2016). A Gaussian-like immersedboundary kernel with three continuous derivatives and improved translational invariance. J. Comput. Phys. 316: 139-144.

Barozzi, G.S., Bussi, C., and Corticelli, M.A. (2004). A fast Cartesian scheme for unsteady heat diffusion on irregular domains. Numer. Heat Tran. Part B: Fund. 46: 59-77.

Bernardini, M., Modesti, D., and Pirozzoli, S. (2016). On the suitability of the immersed boundary method for the simulation of highReynolds-number separated turbulent flows. Comput. \& Fliud 130: 84-93.

Borazjani, I., Ge, L., Le, T., and Sotiropoulos, F. (2013). A parallel overset-curvilinear-immersed boundary framework for simulating complex 3D incompressible flows. Comput. \& Fliud 77: 76-96.

Boukharfane, R., Eugênio Ribeiro, F.H., Bouali, Z., and Mura, A. (2018). A combined ghost-point-forcing/direct-forcing immersed boundary method (IBM) for compressible flow simulations. Comput. \& Fliud 162: 91-112. 
Breugem, W.-P. (2012). A second-order accurate immersed boundary method for fully resolved simulations of particle-laden flows. J. Comput. Phys. 231: 4469-4498.

Carini, M., Giannetti, F., and Auteri, F. (2014). First instability and structural sensitivity of the flow past two side-by-side cylinders. J. Fluid Mech. 749: 627-648.

Cecere, D. and Giacomazzi, E. (2014). An immersed volume method for large Eddy simulation of compressible flows using a staggeredgrid approach. Comput. Meth. Appl. Mech. Eng. 280: 1-27.

Chang, S., laccarino, G., Ham, F., Elkins, C., and Monismith, S. (2014). Local shear and mass transfer on individual coral colonies: computations in unidirectional and wave-driven flows. J. Geophys. Res: Oceans 119: 2599-2619.

Chen, L., Yu, Y., and Hou, G. (2013). Sharp-interface immersed boundary lattice Boltzmann method with reduced spuriouspressure oscillations for moving boundaries. Phys. Rev. 87: 053306.

Chen, L., Yu, Y., Lu, J., and Hou, G. (2014). A comparative study of lattice Boltzmann methods using bounce-back schemes and immersed boundary ones for flow acoustic problems. Int. J. Numer. Meth. Fluid. 74: 439-467.

Cheny, Y. and Botella, O. (2010). The LS-STAG method: a new immersed boundary/level-set method for the computation of incompressible viscous flows in complex moving geometries with good conservation properties. J. Comput. Phys. 229: 1043-1076.

Chhabra, R.P., and Richardson, J.F. (1999). Non-Newtonian flow in the process industries: fundamentals and engineering applications. Butterworth-Heinemann, Oxford, Britain.

Chiu, P.-H., Lin, R.-K., and Sheu, T.W.H. (2010). A differentially interpolated direct forcing immersed boundary method for predicting incompressible Navier-Stokes equations in timevarying complex geometries. J. Comput. Phys. 229: 4476-4500.

Cho, H.W., Ha, M.Y., and Park, Y.G. (2019). Natural convection in a square enclosure with two hot inner cylinders, Part II: the effect of two elliptical cylinders with various aspect ratios in a vertical array. Int. J. Heat Mass Tran. 135: 962-973.

Cho, H.W., Park, Y.G., and Ha, M.Y. (2018). The natural convection in a square enclosure with two hot inner cylinders, Part I: the effect of one elliptical cylinder with various aspect ratios in a vertical array. Int. J. Heat Mass Tran. 125: 815-827.

Choi, C., Cho, H.W., Ha, M.Y., and Yoon, H.S. (2015a). Effect of circular cylinder location on three-dimensional natural convection in a cubical enclosure. J. Mech. Sci. Technol. 29: 1307-1318.

Choi, C., Ha, M.Y., and Park, Y.G. (2015b). Characteristics of thermal convection in a rectangular channel with an inner cold circular cylinder. Int. J. Heat Mass Tran. 84: 955-973.

Choi, C., Jeong, S., Ha, M.Y., and Yoon, H.S. (2014). Effect of a circular cylinder's location on natural convection in a rhombus enclosure. Int. J. Heat Mass Tran. 77: 60-73.

Choi, J.-I., Oberoi, R.C., Edwards, J.R., and Rosati, J.A. (2007). An immersed boundary method for complex incompressible flows. J. Comput. Phys. 224: 757-784.

Chung, M.-H. (2006). Cartesian cut cell approach for simulating incompressible flows with rigid bodies of arbitrary shape. Comput. \& Fliud 35: 607-623.

Crocker, R., Dubief, Y., and Desjardins, O. (2014). A second order thermal and momentum immersed boundary method for conjugate heat transfer in a Cartesian finite volume solver, arXiv preprint arXiv:1411.1004.
Crowe, C.T., Troutt, T.R., and Chung, J.N. (1996). Numerical models for two-phase turbulent flows. Annu. Rev. Fluid Mech. 28: 11-43.

Das, S., Deen, N.G., and Kuipers, J.A.M. (2016). Direct numerical simulation for flow and heat transfer through random open-cell solid foams: development of an IBM based CFD model. Catal. Today 273: 140-150.

Das, S., Deen, N.G., and Kuipers, J.A.M. (2017). A DNS study of flow and heat transfer through slender fixed-bed reactors randomly packed with spherical particles. Chem. Eng. Sci. 160: 1-19.

Das, S., Panda, A., Deen, N.G., and Kuipers, J.A.M. (2018). A sharpinterface Immersed Boundary Method to simulate convective and conjugate heat transfer through highly complex periodic porous structures. Chem. Eng. Sci. 191: 1-18.

Day, M.A. (1990). The no-slip condition of fluid dynamics. Erkenntnis 33: 285-296.

De Jong, J.F., van Sint Annaland, M., and Kuipers, J.A.M. (2012a). Membrane-assisted fluidized beds-part 1: development of an immersed boundary discrete particle model. Chem. Eng. Sci. 84: 814-821.

De Jong, J.F., van Sint Annaland, M., and Kuipers, J.A.M. (2012b). Membrane-assisted fluidized beds-Part 2: numerical study on the hydrodynamics around immersed gas-permeating membrane tubes. Chem. Eng. Sci. 84: 822-833.

de Motta, J.C.B., Costa, P., Derksen, J.J., Peng, C., Wang, L.P., Breugem, W.P., Estivalèzes, J.L., Vincent, S., Climent, E., Fede, P., et al (2019). Assessment of numerical methods for fully resolved simulations of particle-laden turbulent flows. Comput. \& Fliud 179: 1-14.

De Rosis, A., Ubertini, S., and Ubertini, F. (2014). A comparison between the interpolated bounce-back scheme and the immersed boundary method to treat solid boundary conditions for laminar flows in the lattice Boltzmann framework. J. Sci. Comput. 61: 477-489.

Deen, N.G., Kriebitzsch, S.H.L., van der Hoef, M.A., and Kuipers, J.A.M. (2012). Direct numerical simulation of flow and heat transfer in dense fluid-particle systems. Chem. Eng. Sci. 81: 329-344.

Deen, N.G. and Kuipers, J.A.M. (2013). Direct numerical simulation of fluid flow and mass transfer in dense fluid-particle systems. Ind. Eng. Chem. Res. 52: 11266-11274.

Deen, N.G. and Kuipers, J.A.M. (2014a). Direct numerical simulation (DNS) of mass, momentum and heat transfer in dense fluidparticle systems. Curr. Opin. Chem. Eng. 5: 84-89.

Deen, N.G. and Kuipers, J.A.M. (2014b). Direct numerical simulation of fluid flow accompanied by coupled mass and heat transfer in dense fluid-particle systems. Chem. Eng. Sci. 116: 645-656.

Deen, N.G., Peters, E.A.J.F., Padding, J.T., and Kuipers, J.A.M. (2014). Review of direct numerical simulation of fluid-particle mass, momentum and heat transfer in dense gas-solid flows. Chem. Eng. Sci. 116: 710-724.

Delouei, A.A., Nazari, M., Kayhani, M.H., Kang, S.K., and Succi, S. (2016). Non-Newtonian particulate flow simulation: a directforcing immersed boundary-lattice Boltzmann approach. Phys. Stat. Mech. Appl. 447: 1-20.

Delouei, A.A., Nazari, M., Kayhani, M.H., and Succi, S. (2015). Immersed boundary - thermal lattice Boltzmann methods for non-Newtonian flows over a heated cylinder: a comparative study. Commun. Comput. Phys. 18: 489-515.

Dierich, F. and Nikrityuk, P.A. (2013). A numerical study of the impact of surface roughness on heat and fluid flow past a cylindrical particle. Int. J. Therm. Sci. 65: 92-103. 
Eshghinejadfard, A. and Thévenin, D. (2016). Numerical simulation of heat transfer in particulate flows using a thermal immersed boundary lattice Boltzmann method. Int. J. Heat Fluid Flow 60: 31-46.

Fadlun, E.A., Verzicco, R., Orlandi, P., and Mohd-Yusof, J. (2000). Combined immersed-boundary finite-difference methods for three-dimensional complex flow simulations. J. Comput. Phys. 161: 35-60.

Faghri, A. and Zhang, Y. (2006). Transport phenomena in multiphase systems. Elsevier, Cambridge, Massachusetts, USA.

Feldman, Y. (2018a). Oscillatory instability of 2D natural convection flow in a square enclosure with a tandem of vertically aligned cylinders. Fluid Dynam. Res. 50: 051410.

Feldman, Y. (2018b). Semi-implicit direct forcing immersed boundary method for incompressible viscous thermal flow problems: a Schur complement approach. Int. J. Heat Mass Tran. 127: 1267-1283.

Feldman, Y. and Gulberg, Y. (2016). An extension of the immersed boundary method based on the distributed Lagrange multiplier approach. J. Comput. Phys. 322: 248-266.

Feng, Z.-G. and Michaelides, E.E. (2004). The immersed boundarylattice Boltzmann method for solving fluid-particles interaction problems. J. Comput. Phys. 195: 602-628.

Feng, Z.-G. and Michaelides, E.E. (2009). Heat transfer in particulate flows with direct numerical simulation (DNS). Int. J. Heat Mass Tran. 52: 777-786.

Feng, Z.-G. and Musong, S.G. (2014). Direct numerical simulation of heat and mass transfer of spheres in a fluidized bed. Powder Technol. 262: 62-70.

Feng, Z.-G. and Roig, A. (2014). Direct numerical simulation of particle heat and mass transfer in a fluidized bed, V01CT18A004. American Society of Mechanical Engineers, Chicago, Illinois, USA.

Fox, R.O. (2014). On multiphase turbulence models for collisional fluid-particle flows. J. Fluid Mech. 742: 368-424.

Gao, T., Tseng, Y.-H., and Lu, X.-Y. (2007). An improved hybrid Cartesian/immersed boundary method for fluid-solid flows. Int. J. Numer. Meth. Fluid. 55: 1189-1211.

Giannetti, F. and Luchini, P. (2007). Structural sensitivity of the first instability of the cylinder wake. J. Fluid Mech. 581: 167-197.

Gilmanov, A. and Sotiropoulos, F. (2005). A hybrid Cartesian/ immersed boundary method for simulating flows with 3D, geometrically complex, moving bodies. J. Comput. Phys. 207: 457-492.

Gilmanov, A., Sotiropoulos, F., and Balaras, E. (2003). A general reconstruction algorithm for simulating flows with complex 3D immersed boundaries on Cartesian grids. J. Comput. Phys. 191: 660-669.

Glowinski, R., Pan, T.W., Hesla, T.I., and Joseph, D.D. (1999). A distributed Lagrange multiplier/fictitious domain method for particulate flows. Int. J. Multiphas. Flow 25: 755-794.

Goldstein, D., Handler, R., and Sirovich, L. (1993). Modeling a No-slip flow boundary with an external force field. J. Comput. Phys. 105: 354-366.

Gong, X., Gong, Z., and Huang, H. (2014). An immersed boundary method for mass transfer across permeable moving interfaces. J. Comput. Phys. 278: 148-168.

Gunn, D.J. (1978). Transfer of heat or mass to particles in fixed and fluidised beds. Int. J. Heat Mass Tran. 21: 467-476.
Höhne, T. and Mehlhoop, J.-P. (2014). Validation of closure models for interfacial drag and turbulence in numerical simulations of horizontal stratified gas-liquid flows. Int. J. Multiphas. Flow 62: 1-16.

Haeri, S. and Shrimpton, J.S. (2012). On the application of immersed boundary, fictitious domain and body-conformal mesh methods to many particle multiphase flows. Int. J. Multiphas. Flow 40: 38-55.

He, L. and Tafti, D.K. (2017). Heat transfer in an assembly of ellipsoidal particles at low to moderate Reynolds numbers. Int. J. Heat Mass Tran. 114: 324-336.

Horne, W.J. and Mahesh, K. (2019a). A massively-parallel, unstructured overset method for mesh connectivity. J. Comput. Phys. 376: 585-596.

Horne, W.J. and Mahesh, K. (2019b). A massively-parallel, unstructured overset method to simulate moving bodies in turbulent flows. J. Comput. Phys. 397: 108790.

Hosseinverdi, S. and Fasel, H.F. (2017). 23rd AIAA computational fluid dynamics conference. American Institute of Aeronautics and Astronautics, Denver, Colorado, p. 3624.

Hu, H.H., Joseph, D.D., and Crochet, M.J. (1992). Direct simulation of fluid particle motions. Theor. Comput. Fluid Dynam. 3: 285-306.

Hu, O., Zhao, N., and Liu, J.M. (2013). A ghost cell method for turbulent compressible viscous flows on adaptive Cartesian grids. Proc. Eng. 67: 241-249.

Hu, W.-F., Lai, M.-C., and Misbah, C. (2018). A coupled immersed boundary and immersed interface method for interfacial flows with soluble surfactant. Comput. \& Fliud 168: 201-215.

Huang, H., Sugiyama, K., and Takagi, S. (2009). An immersed boundary method for restricted diffusion with permeable interfaces. J. Comput. Phys. 228: 5317-5322.

Huang, W.-X., and Tian, F.-B. (2019). Recent trends and progresses in the immersed boundary method. Proc. IME C J. Mech. Eng. Sci. 233: 7617-7636.

laccarino, G. and Moreau, S. (2005). Natural and forced conjugate heat transfer in complex geometries on Cartesian adapted grids. J. Fluid Eng. 128: 838-846.

laccarino, G., Ooi, A., Durbin, P.A., and Behnia, M. (2002). Conjugate heat transfer predictions in two-dimensional ribbed passages. Int. J. Heat Fluid Flow 23: 340-345.

Iaccarino, G. and Verzicco, R. (2003). Immersed boundary technique for turbulent flow simulations. Appl. Mech. Rev. 56: 331-347.

Jafari, S., Yamamoto, R., and Rahnama, M. (2011). Lattice-Boltzmann method combined with smoothed-profile method for particulate suspensions. Phys. Rev. 83: 026702.

Jahanshaloo, L., Sidik, N.A.C., Fazeli, A., and HA, M.P. (2016). An overview of boundary implementation in lattice Boltzmann method for computational heat and mass transfer. Int. Commun. Heat Mass Tran. 78: 1-12.

Jayathilake, P.G., Liu, G., Tan, Z., and Khoo, B.C. (2011). Numerical study of a permeable capsule under Stokes flows by the immersed interface method. Chem. Eng. Sci. 66: 2080-2090.

Jayathilake, P.G., Tan, Z., Khoo, B.C., and Wijeysundera, N.E. (2010). Deformation and osmotic swelling of an elastic membrane capsule in Stokes flows by the immersed interface method. Chem. Eng. Sci. 65: 1237-1252.

Jeong, J. and Hussain, F. (1995). On the identification of a vortex. J. Fluid Mech. 285: 69-94.

Jiang, Y., Liang, A., Sun, X., and Jing, X. (2012). Multi-domain spectral immersed interface method for solving elliptic equation with a 
global description of discontinuous functions. Chin. J. Aeronaut. 25: 297-310.

Johnson, A.A. and Tezduyar, T.E. (1999). Advanced mesh generation and update methods for 3D flow simulations. Comput. Mech. 23: 130-143.

Kadoch, B., Kolomenskiy, D., Angot, P., and Schneider, K. (2012). A volume penalization method for incompressible flows and scalar advection-diffusion with moving obstacles. J. Comput. Phys. 231: 4365-4383.

Kajishima, T., Takiguchi, S., Hamasaki, H., and Miyake, Y. (2001). Turbulence structure of particle-laden flow in a vertical plane channel due to vortex shedding. JSME Int. J. Ser. B Fluids Therm. Eng. 44: 526-535.

Kang, S., laccarino, G., and Ham, F. (2009). DNS of buoyancydominated turbulent flows on a bluff body using the immersed boundary method. J. Comput. Phys. 228: 3189-3208.

Kang, S.K. and Hassan, Y.A. (2010). A comparative study of interface schemes in the immersed boundary method for a moving solid boundary problem using the lattice Boltzmann method. American Society of Mechanical Engineers, Montreal, Quebec, Canada, pp. 2433-2440.

Kang, S.K. and Hassan, Y.A. (2011). A comparative study of directforcing immersed boundary-lattice Boltzmann methods for stationary complex boundaries. Int. J. Numer. Meth. Fluid. 66: $1132-1158$.

Kedem, O. and Katchalsky, A. (1958). Thermodynamic analysis of the permeability of biological membranes to non-electrolytes. Biochim. Biophys. Acta 27: 229-246.

Kedia, K.S., Safta, C., Ray, J., Najm, H.N., and Ghoniem, A.F. (2014). A second-order coupled immersed boundary-SAMR construction for chemically reacting flow over a heat-conducting Cartesian grid-conforming solid. J. Comput. Phys. 272: 408-428.

Kee, R.J., Coltrin, M.E., and Glarborg, P. (2005). Chemically reacting flow: theory and practice. John Wiley \& Sons, Hoboken, New Jersey.

Kempe, T. and Fröhlich, J. (2012). An improved immersed boundary method with direct forcing for the simulation of particle laden flows. J. Comput. Phys. 231: 3663-3684.

Kempe, T., Lennartz, M., Schwarz, S., and Fröhlich, J. (2015). Imposing the free-slip condition with a continuous forcing immersed boundary method. J. Comput. Phys. 282: 183-209.

Kirkpatrick, M.P. and Armfield, S.W. (2003). A representation of curved boundaries for the solution of the Navier-Stokes equations on a staggered three-dimensional Cartesian grid. J. Comput. Phys.: 1-36, https://doi.org/10.1016/s0021-9991(02) 00013-x.

Kim, B.S., Lee, D.S., Ha, M.Y., and Yoon, H.S. (2008). A numerical study of natural convection in a square enclosure with a circular cylinder at different vertical locations. Int. J. Heat Mass Tran. 51: 1888-1906.

Kim, D. and Choi, H. (2006). Immersed boundary method for flow around an arbitrarily moving body. J. Comput. Phys. 212: 662-680.

Kim, J. and Choi, H.C. (2004). An immersed-boundary finite-volume method for simulation of heat transfer in complex geometries. KSME Int. J. 18: 1026-1035.

Kim, J., Kim, D., and Choi, H. (2001). An immersed-boundary finitevolume method for simulations of flow in complex geometries. J. Comput. Phys. 171: 132-150.

Kim, Y. and Peskin, C.S. (2007). Penalty immersed boundary method for an elastic boundary with mass. Phys. Fluids 19: 053103.
Kim, Y. and Peskin, C.S. (2009). 3-D parachute simulation by the immersed boundary method. Comput. \& Fliud 38: 1080-1090.

Kriebitzsch, S.H.L., van der Hoef, M.A., and Kuipers, J.A.M. (2013). Drag force in discrete particle models-continuum scale or single particle scale? AIChE J. 59: 316-324.

Kumar, M. and Roy, S. (2016). A sharp interface immersed boundary method for moving geometries with mass conservation and smooth pressure variation. Comput. \& Fliud 137: 15-35.

Lai, M.-C. and Li, Z. (2001). A remark on jump conditions for the threedimensional Navier-Stokes equations involving an immersed moving membrane. Appl. Math. Lett. 14: 149-154.

Lai, M.-C. and Peskin, C.S. (2000). An immersed boundary method with formal second-order accuracy and reduced numerical viscosity. J. Comput. Phys. 160: 705-719.

Lambert, R.A., Picano, F., Breugem, W.-P., and Brandt, L. (2013). Active suspensions in thin films: nutrient uptake and swimmer motion. J. Fluid Mech. 733: 528-557.

Lashgari, I., Picano, F., Breugem, W.-P., and Brandt, L. (2014). Laminar, turbulent, and inertial shear-thickening regimes in channel flow of neutrally buoyant particle suspensions. Phys. Rev. Lett. 113: 254502.

Lauga, E., Brenner, M., and Stone, H. (2007). Microfluidics: the no-slip boundary condition. In: Tropea, C., Yarin, A.L., and Foss, J.F. (Eds.), Springer handbook of experimental fluid mechanics. Springer Handbooks, Springer, Berlin, Heidelberg, pp. 1219-1240. https://doi.org/10.1007/978-3-540-30299-5_19.

Le, D.V., Khoo, B.C., and Lim, K.M. (2008). An implicit-forcing immersed boundary method for simulating viscous flows in irregular domains. Comput. Methods Appl. Mech. Eng. 197: 2119-2130.

Le, D.V., Khoo, B.C., and Peraire, J. (2006). An immersed interface method for viscous incompressible flows involving rigid and flexible boundaries. J. Comput. Phys. 220: 109-138.

Lee, H.G. and Kim, J. (2012). Regularized Dirac delta functions for phase field models. Int. J. Numer. Meth. Eng. 91: 269-288.

Lee, J., Kim, J., Choi, H., and Yang, K.-S. (2011). Sources of spurious force oscillations from an immersed boundary method for moving-body problems. J. Comput. Phys. 230: 2677-2695.

Lee, J. and You, D. (2013). An implicit ghost-cell immersed boundary method for simulations of moving body problems with control of spurious force oscillations. J. Comput. Phys. 233: 295-314.

Lee, L. and LeVeque, R. (2003). An immersed interface method for incompressible Navier-Stokes equations. SIAM J. Sci. Comput. 25: 832 .

Lee, S.H., Seo, Y.M., Yoon, H.S., and Ha, M.Y. (2016). Threedimensional natural convection around an inner circular cylinder located in a cubic enclosure with sinusoidal thermal boundary condition. Int. J. Heat Mass Tran. 101: 807-823.

Leveque, R.J. and Li, Z. (1994). The immersed interface method for elliptic equations with discontinuous coefficients and singular sources. SIAM J. Numer. Anal. 31: 1019-1044.

LeVeque, R.J. and Li, Z. (1997). Immersed interface methods for Stokes flow with elastic boundaries or surface tension. SIAM J. Sci. Comput. 18: 709-735.

Li, Y., Jung, E., Lee, W., Lee, H.G., and Kim, J. (2012). Volume preserving immersed boundary methods for two-phase fluid flows. Int. J. Numer. Meth. Fluid. 69: 842-858.

Li, Z., Jaiman, R.K., and Khoo, B.C. (2016). An immersed interface method for flow past circular cylinder in the vicinity of a plane moving wall. Int. J. Numer. Meth. Fluid. 81: 611-639. 
Li, Z. and Lai, M.-C. (2001). The immersed interface method for the Navier-Stokes equations with singular forces. J. Comput. Phys. 171: 822-842.

Liang, A., Jing, X., and Sun, X. (2008). Constructing spectral schemes of the immersed interface method via a global description of discontinuous functions. J. Comput. Phys. 227: 8341-8366.

Liao, C.-C., Chang, Y.-W., Lin, C.-A., and McDonough, J.M. (2010). Simulating flows with moving rigid boundary using immersedboundary method. Comput. \& Fliud 39: 152-167.

Liao, C.-C. and Lin, C.-A. (2012a). Influences of a confined elliptic cylinder at different aspect ratios and inclinations on the laminar natural and mixed convection flows. Int. J. Heat Mass Tran. 55: 6638-6650.

Liao, C.-C. and Lin, C.-A. (2012b). Simulations of natural and forced convection flows with moving embedded object using immersed boundary method. Comput. Methods Appl. Mech. Eng. 213-216: 58-70.

Linnick, M.N. and Fasel, H.F. (2005). A high-order immersed interface method for simulating unsteady incompressible flows on irregular domains. J. Comput. Phys. 204: 157-192.

Liu, X., Song, F., and Xu, C. (2019). An efficient spectral method for the inextensible immersed interface in incompressible flows. Commun. Comput. Phys. 25: 1071-1096.

Lo, D.C., Lee, C.P., and Lin, I.F. (2018). An efficient immersed boundary method for fluid flow simulations with moving boundaries. Appl. Math. Comput. 328: 312-337.

Lu, J., Das, S., Peters, E., and Kuipers, J.A.M. (2018a). Direct numerical simulation of fluid flow and mass transfer in dense fluid-particle systems with surface reactions. Chem. Eng. Sci. 176: 1-18.

Lu, J., Peters, E., and Kuipers, J.A.M. (2019a). Direct numerical simulation of fluid flow and dependently coupled heat and mass transfer in fluid-particle systems. Chem. Eng. Sci. 204: 203-219.

Lu, J., Peters, E.A.J.F., and Kuipers, J.A.M. (2018b). Direct numerical simulation of fluid flow and mass transfer in particle clusters. Ind. Eng. Chem. Res. 57: 4664-4679.

Lu, J., Tan, M.D., Peters, E.A.J.F., and Kuipers, J.A.M. (2018c). Direct numerical simulation of reactive fluid-particle systems using an immersed boundary method. Ind. Eng. Chem. Res. 57: 15565-15578.

Lu, J., Zhu, X., Peters, E.A.J.F., Verzicco, R., Lohse, D., and Kuipers, J.A.M. (2019b). Moving from momentum transfer to heat transfer - a comparative study of an advanced Graetz-Nusselt problem using immersed boundary methods. Chem. Eng. Sci. 198: 317-333.

Luo, H., Dai, H., Ferreira De Sousa, P.J.S.A., and Yin, B. (2012). On the numerical oscillation of the direct-forcing immersed-boundary method for moving boundaries. Comput. \& Fliud 56: 61-76.

Luo, H., Mittal, R., Zheng, X., Bielamowicz, S.A., Walsh, R.J., and Hahn, J.K. (2008). An immersed-boundary method for flow structure interaction in biological systems with application to phonation. J. Comput. Phys. 227: 9303-9332.

Luo, K., Mao, C., Fan, J., Zhuang, Z., and Haugen, N.E.L. (2018). Fully resolved simulations of single char particle combustion using a ghost-cell immersed boundary method. AIChE J. 64: 2851-2863.

Luo, K., Mao, C., Zhuang, Z., Fan, J., and Haugen, N.E.L. (2017). A ghostcell immersed boundary method for the simulations of heat transfer in compressible flows under different boundary conditions Part-II: complex geometries. Int. J. Heat Mass Tran. 104: 98-111.

Luo, K., Tan, J., Wang, Z., and Fan, J. (2016a). Particle-resolved direct numerical simulation of gas-solid dynamics in experimental fluidized beds. AIChE J. 62: 1917-1932.
Luo, K., Wang, Z., and Fan, J. (2007a). A modified immersed boundary method for simulations of fluid-particle interactions. Comput. Meth. Appl. Mech. Eng. 197: 36-46.

Luo, K., Wang, Z., and Fan, J. (2010). Response of force behaviors of a spherical particle to an oscillating flow. Phys. Lett. A 374: 3046-3052.

Luo, K., Wang, Z., Fan, J., and Cen, K. (2007b). Full-scale solutions to particle-laden flows: multidirect forcing and immersed boundary method. Phys. Rev. 76: 066709.

Luo, K., Wang, D., Jin, T., Wang, S., Wang, Z., Tan, J., and Fan, J. (2020). Analysis and development of novel data-driven drag models based on direct numerical simulations of fluidized beds. Authorea, https://doi.org/10.1016/j.ces.2020.116245.

Luo, K., Wang, Z., Tan, J., and Fan, J. (2019). An improved direct-forcing immersed boundary method with inward retraction of Lagrangian points for simulation of particle-laden flows. J. Comput. Phys. 376: 210-227.

Luo, K., Wei, A., Wang, Z., and Fan, J. (2013). Fully-resolved DNS study of rotation behaviors of one and two particles settling near a vertical wall. Powder Technol. 245: 115-125.

Luo, K., Zhuang, Z., Fan, J., and Haugen, N.E.L. (2016b). A ghost-cell immersed boundary method for simulations of heat transfer in compressible flows under different boundary conditions. Int. J. Heat Mass Tran. 92: 708-717.

Lupo, G., Niazi Ardekani, M., Brandt, L., and Duwig, C. (2019). An Immersed Boundary Method for flows with evaporating droplets. Int. J. Heat Mass Tran. 143: 118563.

Makino, A., Namikiri, T., and Kimura, K. (2003). Combustion rates of graphite rods in the forward stagnation field with hightemperature airflow. Combust. Flame 132: 743-753.

Mark, A., Svenning, E., and Edelvik, F. (2013). An immersed boundary method for simulation of flow with heat transfer. Int. J. Heat Mass Tran. 56: 424-435.

Mark, A. and van Wachem, B.G.M. (2008). Derivation and validation of a novel implicit second-order accurate immersed boundary method. J. Comput. Phys. 227: 6660-6680.

Maxey, M. (2017). Simulation methods for particulate flows and concentrated suspensions. Annu. Rev. Fluid Mech. 49: 171-193.

McGurn, M.T., Ruggirello, K.P., and DesJardin, P.E. (2013). An Eulerian-Lagrangian moving immersed interface method for simulating burning solids. J. Comput. Phys. 241: 364-387.

Mehrabadi, M., Murphy, E., and Subramaniam, S. (2016). Development of a gas-solid drag law for clustered particles using particle-resolved direct numerical simulation. Chem. Eng. Sci. 152: 199-212.

Merlin, C., Domingo, P., and Vervisch, L. (2013). Immersed boundaries in large eddy simulation of compressible flows. Flow, Turbul. Combust. 90: 29-68.

Meyer, M., Devesa, A., Hickel, S., Hu, X.Y., and Adams, N.A. (2010). A conservative immersed interface method for Large-Eddy Simulation of incompressible flows. J. Comput. Phys. 229: 6300-6317.

Michaels, D. and Ghoniem, A.F. (2016). Impact of the bluff-body material on the flame leading edge structure and flame-flow interaction of premixed CH4/air flames. Combust. Flame 172: 62-78.

Mino, Y., Shinto, H., Sakai, S., and Matsuyama, H. (2017). Effect of internal mass in the lattice Boltzmann simulation of moving solid bodies by the smoothed-profile method. Phys. Rev. 95: 043309. 
Mittal, R., Dong, H., Bozkurttas, M., Najjar, F.M., Vargas, A., and von Loebbecke, A. (2008). A versatile sharp interface immersed boundary method for incompressible flows with complex boundaries. J. Comput. Phys. 227: 4825-4852.

Mittal, R. and laccarino, G. (2005). Immersed boundary methods. Annu. Rev. Fluid Mech. 37: 239-261.

Miyauchi, S., Takeuchi, S. and Kajishima, T. (2017). A numerical method for interaction problems between fluid and membranes with arbitrary permeability for fluid. J. Comput. Phys. 345: 33-57.

Mohapatra, P., Dubey, A., Daley, C., Vanella, M., and Balaras, E. (2013). Parallel algorithms for using Lagrangian markers in immersed boundary method with adaptive mesh refinement in FLASH. In: 2013 25th International symposium on computer architecture and high performance computing. IEEEde Galinhas, PortoPernambuco, Brazil, pp. 214-220.

Mohd-Yusof, J. (1997). Combined immersed-boundary/B-spline methods for simulations of flow in complex geometries. In Annual research briefs. NASA Ames Research Center $=$ Stanford University Center of Turbulence Research, Stanford, pp. 317-327.

Muldoon, F. and Acharya, S. (2008). A divergence-free interpolation scheme for the immersed boundary method. Int. J. Numer. Meth. Fluid. 56: 1845-1884.

Muralidharan, B. and Menon, S. (2016). A high-order adaptive Cartesian cut-cell method for simulation of compressible viscous flow over immersed bodies. J. Comput. Phys. 321: 342-368.

Muralidharan, B. and Menon, S. (2018). Simulation of moving boundaries interacting with compressible reacting flows using a second-order adaptive Cartesian cut-cell method. J. Comput. Phys. 357: 230-262.

Musielak, M.M., Karp-Boss, L., Jumars, P.A., and Fauci, L.J. (2009). Nutrient transport and acquisition by diatom chains in a moving fluid. J. Fluid Mech. 638: 401-421.

Musong, S.G., Feng, Z.-G., Michaelides, E.E., and Mao, S. (2015). Application of a three-dimensional immersed boundary method for free convection from single spheres and aggregates. J. Fluid Eng. 138: 041304-04-10.

Nagendra, K., Tafti, D.K., and Viswanath, K. (2014). A new approach for conjugate heat transfer problems using immersed boundary method for curvilinear grid based solvers. J. Comput. Phys. 267: 225-246.

Nakayama, Y. and Yamamoto, R. (2005). Simulation method to resolve hydrodynamic interactions in colloidal dispersions. Phys. Rev. 71: 036707

Naumann, Z. and Schiller, L. (1935). A drag coefficient correlation. Z Ver Deutsch Ing 77: 318-323.

Pacheco-Vega, A., Pacheco, J.R., and Rodic, T. (2007). A general scheme for the boundary conditions in convective and diffusive heat transfer with immersed boundary methods. J. Heat Tran. 129: 1506.

Pacheco, J.R., Pacheco-Vega, A., Rodic, T., and Peck, R.E. (2005). Numerical simulations of heat transfer and fluid flow problems using an immersed-boundary finite-volume method on nonstaggered grids. Numer. Heat Tran. Part B: Fund. 48: 1-24.

Pan, D. (2010). A simple and accurate ghost cell method for the computation of incompressible flows over immersed bodies with heat transfer. Numer. Heat Tran. Part B: Fund. 58: 17-39.

Pan, D. (2012). A general boundary condition treatment in immersed boundary methods for incompressible Navier-Stokes equations with heat transfer. Numer. Heat Tran. Part B: Fund. 61: 279-297.
Pan, D. and Shen, T.-T. (2009). Computation of incompressible flows with immersed bodies by a simple ghost cell method. Int. J. Numer. Meth. Fluid. 60: 1378-1401.

Pandey, S., Park, Y.G., and Ha, M.Y. (2019). An exhaustive review of studies on natural convection in enclosures with and without internal bodies of various shapes. Int. J. Heat Mass Tran. 138: 762-795.

Park, H.K., Ha, M.Y., Yoon, H.S., Park, Y.G., and Son, C. (2013a). A numerical study on natural convection in an inclined square enclosure with a circular cylinder. Int. J. Heat Mass Tran. 66: 295-314.

Park, Y.G., Ha, M.Y., and Yoon, H.S. (2013b). Study on natural convection in a cold square enclosure with a pair of hot horizontal cylinders positioned at different vertical locations. Int. J. Heat Mass Tran. 65: 696-712.

Paul, I., Arul Prakash, K., and Vengadesan, S. (2013). Forced convective heat transfer from unconfined isothermal and isoflux elliptic cylinders. Numer. Heat Tran. Part A: Appl. 64: 648-675.

Perelman, T.L. (1961). On conjugated problems of heat transfer. Int. J. Heat Mass Tran. 3: 293-303.

Peskin, C.S. (1972). Flow patterns around heart valves: a numerical method. J. Comput. Phys. 10: 252-271.

Peskin, C.S. (1977). Numerical analysis of blood flow in the heart. J. Comput. Phys. 25: 220-252.

Peskin, C.S. (2002). The immersed boundary method. Acta Numer. 11: 479-517.

Picano, F., Breugem, W.-P., and Brandt, L. (2015). Turbulent channel flow of dense suspensions of neutrally buoyant spheres. J. Fluid Mech. 764: 463-487.

Pralits, J.O., Giannetti, F., and Brandt, L. (2013). Three-dimensional instability of the flow around a rotating circular cylinder. J. Fluid Mech. 730: 5-18.

Qu, S. and You, C. (2019). Direct numerical simulation (DNS) of alkali metals released during char combustion. Fuel 255: 115763.

Rai, M.M. and Moin, P. (1991). Direct simulations of turbulent flow using finite-difference schemes. J. Comput. Phys. 96: 15-53.

Rao, I.J. and Rajagopal, K.R. (1999). The effect of the slip boundary condition on the flow of fluids in a channel. Acta Mech. 135: 113-126.

Rejniak, K.A. (2007). An immersed boundary framework for modelling the growth of individual cells: an application to the early tumour development. J. Theor. Biol. 247: 186-204.

Rejniak, K.A. and Dillon, R.H. (2007). A single cell-based model of the ductal tumour microarchitecture. Comput. Math. Meth. Med. 8: 51-69.

Rejniak, K.A., Kliman, H.J., and Fauci, L.J. (2004). A computational model of the mechanics of growth of the villous trophoblast bilayer. Bull. Math. Biol. 66: 199-232.

Ren, W., Shu, C., and Yang, W. (2013). An efficient immersed boundary method for thermal flow problems with heat flux boundary conditions. Int. J. Heat Mass Tran. 64: 694-705.

Roghair, I., Annaland, M.V.S., and Kuipers, J. (2016). An improved front-tracking technique for the simulation of mass transfer in dense bubbly flows. Chem. Eng. Sci. 152: 351-369.

Roma, A.M., Peskin, C.S., and Berger, M.J. (1999). An adaptive version of the immersed boundary method. J. Comput. Phys. 153: 509-534.

Rzehak, R. and Krepper, E. (2013). Closure models for turbulent bubbly flows: a CFD study. Nucl. Eng. Des. 265: 701-711. 
Saadat, A., Guido, C.J., laccarino, G., and Shaqfeh, E.S.G. (2018). Immersedfinite-element method for deformable particle suspensions in viscous and viscoelastic media. Phys. Rev. 98: 063316.

Saiki, E.M. and Biringen, S. (1996). Numerical simulation of a cylinder in uniform flow: application of a virtual boundary method. J. Comput. Phys. 123: 450-465.

Santarelli, C., Fröhlich, J., and Kempe, T. (2016). Immersed boundary methods for heat transfer. Int. J. Numer. Meth. Heat Fluid Flow 26: 504-514.

Sato, N., Takeuchi, S., Kajishima, T., Inagaki, M., and Horinouchi, N. (2016). A consistent direct discretization scheme on Cartesian grids for convective and conjugate heat transfer. J. Comput. Phys. 321: 76-104.

Schneiders, L., Hartmann, D., Meinke, M., and Schröder, W. (2013). An accurate moving boundary formulation in cut-cell methods. J. Comput. Phys. 235: 786-809.

Seifert, U. (1991). Adhesion of vesicles in two dimensions. Phys. Rev. 43: 6803

Seo, J.H. and Mittal, R. (2011a). A high-order immersed boundary method for acoustic wave scattering and low-Mach number flowinduced sound in complex geometries. J. Comput. Phys. 230: 1000-1019.

Seo, J.H. and Mittal, R. (2011b). A sharp-interface immersed boundary method with improved mass conservation and reduced spurious pressure oscillations. J. Comput. Phys. 230: 7347-7363.

Seo, Y.M., Doo, J.H., and Ha, M.Y. (2016). Three-dimensional flow instability of natural convection induced by variation in radius of inner circular cylinder inside cubic enclosure. Int. J. Heat Mass Tran. 95: 566-578.

Seo, Y.M., Ha, M.Y., and Park, Y.G. (2019a). A numerical study on the three-dimensional natural convection with a cylinder in a long rectangular enclosure. Part I: size effect of a circular cylinder or an elliptical cylinder. Int. J. Heat Mass Tran. 134: 420-436.

Seo, Y.M., Ha, M.Y., and Park, Y.G. (2019b). A numerical study on the three-dimensional natural convection with a cylinder in a long rectangular enclosure. Part II: inclination angle effect of the elliptical cylinder. Int. J. Heat Mass Tran. 131: 795-806.

Shinn, A.F., Goodwin, M.A., and Vanka, S.P. (2009). Immersed boundary computations of shear-and buoyancy-driven flows in complex enclosures. Int. J. Heat Mass Tran. 52: 4082-4089, \%। 2013-10-27 19:15:00

Silva, A.L.F.L.E., Silveira-Neto, A., and Damasceno, J.J.R. (2003). Numerical simulation of two-dimensional flows over a circular cylinder using the immersed boundary method. J. Comput. Phys. 189: 351-370.

Sotiropoulos, F. and Borazjani, I. (2009). A review of state-of-the-art numerical methods for simulating flow through mechanical heart valves. Med. Biol. Eng. Comput. 47: 245-256.

Sotiropoulos, F. and Yang, X. (2014). Immersed boundary methods for simulating fluid structure interaction. Prog. Aero. Sci. 65: 1-21.

Spizzichino, A., Goldring, S., and Feldman, Y. (2019a). The immersed boundary method: application to two-phase immiscible flows. Commun. Comput. Phys. 25: 107-134.

Spizzichino, A., Zemach, E., and Feldman, Y. (2019b). Oscillatory instability of a 3D natural convection flow around a tandem of cold and hot vertically aligned cylinders placed inside a cold cubic enclosure. Int. J. Heat Mass Tran. 141: 327-345.

Stockie, J.M. (1997). Analysis and computation of immersed boundaries, with application to pulp fibres. University of British
Columbia. Retrieved from https://open.library.ubc.ca/ collections/ubctheses/831/items/1.0080530.

Su, S.-W., Lai, M.-C., and Lin, C.-A. (2007). An immersed boundary technique for simulating complex flows with rigid boundary. Comput. \& Fliud 36: 313-324.

Sulaiman, M., Hammouti, A., Climent, E., and Wachs, A. (2019). Coupling the fictitious domain and sharp interface methods for the simulation of convective mass transfer around reactive particles: towards a reactive Sherwood number correlation for dilute systems. Chem. Eng. Sci. 198: 334-351.

Sun, B., Tenneti, S., and Subramaniam, S. (2015). Modeling average gas-solid heat transfer using particle-resolved direct numerical simulation. Int. J. Heat Mass Tran. 86: 898-913.

Suzuki, K., Kawasaki, T., Furumachi, N., Tai, Y., and Yoshino, M. (2018). A thermal immersed boundary-lattice Boltzmann method for moving-boundary flows with Dirichlet and Neumann conditions. Int. J. Heat Mass Tran. 121: 1099-1117.

Takeuchi, S., Tazaki, A., Miyauchi, S., and Kajishima, T. (2019). A relation between membrane permeability and flow rate at low Reynolds number in circular pipe. J. Membr. Sci. 582: 91-102.

Takeuchi, S., Tsutsumi, T., and Kajishima, T. (2013). Effect of temperature gradient within a solid particle on the rotation and oscillation modes in solid-dispersed two-phase flows. Int. J. Heat Fluid Flow 43: 15-25.

Takeuchi, S., Tsutsumi, T., Kondo, K., Harada, T., and Kajishima, T. (2015). Heat transfer in natural convection with finite-sized particles considering thermal conductance due to inter-particle contacts. Comput. Therm. Sci. 7: 385-404.

Tan, Z., Le, D.V., Lim, K.M., and Khoo, B.C. (2009). An immersed interface method for the incompressible Navier-Stokes equations with discontinuous viscosity across the interface. SIAM J. Sci. Comput. 31: 1798-1819.

Tang, Y., Kriebitzsch, S.H.L., Peters, E.A.J.F., van der Hoef, M.A., and Kuipers, J.A.M. (2014). A methodology for highly accurate results of direct numerical simulations: drag force in dense gas solid flows at intermediate Reynolds number. Int. J. Multiphas. Flow 62: 73-86.

Tang, Y., Lau, Y.M., Deen, N.G., Peters, E.A.J.F., and Kuipers, J.A.M. (2016). Direct numerical simulations and experiments of a pseudo-2D gas-fluidized bed. Chem. Eng. Sci. 143: 166-180.

Tang, Y.Y., Peters, E.F., Kuipers, J.A.M.H., Kriebitzsch, S.H.L.S., and van der Hoef, M.A.M. (2015). A new drag correlation from fully resolved simulations of flow past monodisperse static arrays of spheres. AIChE J. 61: 688-698.

Tavassoli, H., Kriebitzsch, S.H.L., van der Hoef, M.A., Peters, E.A.J.F., and Kuipers, J.A.M. (2013). Direct numerical simulation of particulate flow with heat transfer. Int. J. Multiphas. Flow 57: 29-37.

Tenneti, S., Garg, R., and Subramaniam, S. (2011). Drag law for monodisperse gas-solid systems using particle-resolved direct numerical simulation of flow past fixed assemblies of spheres. Int. J. Multiphas. Flow 37: 1072-1092.

Tenneti, S., Sun, B., Garg, R., and Subramaniam, S. (2013). Role of fluid heating in dense gas-solid flow as revealed by particleresolved direct numerical simulation. Int. J. Heat Mass Tran. 58: 471-479.

Tezduyar, T.E., Behr, M., Mittal, S., and Liou, J. (1992). A new strategy for finite element computations involving moving boundaries and interfaces-the deforming-spatial-domain/space-time procedure: II. Computation of free-surface flows, two-liquid 
flows, and flows with drifting cylinders. Comput. Methods Appl. Mech. Eng. 94: 353-371.

Tseng, Y.-H. and Ferziger, J.H. (2003). A ghost-cell immersed boundary method for flow in complex geometry. J. Comput. Phys. 192: 593-623.

Tsutsumi, T., Takeuchi, S., and Kajishima, T. (2014). Heat transfer and particle behaviours in dispersed two-phase flow with different heat Conductivities for liquid and solid. Flow, Turbul. Combust. 92: 103-119.

Udaykumar, H.S., Kan, H.-C., Shyy, W., and Tran-Son-Tay, R. (1997). Multiphase dynamics in arbitrary geometries on fixed Cartesian grids. J. Comput. Phys. 137: 366-405.

Udaykumar, H.S., Mittal, R., Rampunggoon, P., and Khanna, A (2001). A sharp interface Cartesian grid method for simulating flows with complex moving boundaries. J. Comput. Phys. 174: 345-380.

Uhlmann, M. (2005). An immersed boundary method with direct forcing for the simulation of particulate flows. J. Comput. Phys. 209: 448-476.

Uhlmann, M. and Doychev, T. (2014). Sedimentation of a dilute suspension of rigid spheres at intermediate Galileo numbers: the effect of clustering upon the particle motion. J. Fluid Mech. 752: 310-348.

Umphrey, C., DeLeon, R., and Senocak, I. (2017). Direct numerical simulation of turbulent katabatic slope flows with an immersedboundary method. Boundary-Layer Meteorol. 164: 367-382.

Unverdi, S.O. and Tryggvason, G. (1992). A front-tracking method for viscous, incompressible, multi-fluid flows. J. Comput. Phys. 100: 25-37.

Wang, Z., Fan, J., and Luo, K. (2008). Combined multi-direct forcing and immersed boundary method for simulating flows with moving particles. Int. J. Multiphas. Flow 34: 283-302.

Wang, Z., Fan, J., Luo, K., and Cen, K. (2009). Immersed boundary method for the simulation of flows with heat transfer. Int. J. Heat Mass Tran. 52: 4510-4518.

Wu, J., Cheng, Y., and Miller, L.A. (2017). An iterative source correction based immersed boundary-lattice Boltzmann method for thermal flow simulations. Int. J. Heat Mass Tran. 115: 450-460.

Xia, J., Luo, K., and Fan, J. (2014). A ghost-cell based high-order immersed boundary method for inter-phase heat transfer simulation. Int. J. Heat Mass Tran. 75: 302-312.

Xia, J., Luo, K., and Fan, J. (2015). Simulating heat transfer from moving rigid bodies using high-order ghost-cell based immersedboundary method. Int. J. Heat Mass Tran. 89: 856-865.

Xia, J., Luo, K., and Fan, J. (2017). Fully resolved numerical simulation of interphase heat transfer in gas-solid turbulent flow. Int. J. Heat Mass Tran. 112: 45-60.

Xia, J., Luo, K., and Fan, J. (2018). Fully resolved direct numerical simulation of multiphase turbulent thermal boundary layer with finite size particles. Int. J. Multiphas. Flow 99: 454-466.

Xu, S. (2008). The immersed interface method for simulating prescribed motion of rigid objects in an incompressible viscous flow. J. Comput. Phys. 227: 5045-5071.

$\mathrm{Xu}, \mathrm{S}$. (2009). Singular forces in the immersed interface method for rigid objects in 3D. Appl. Math. Lett. 22: 827-833.

$\mathrm{Xu}, \mathrm{S}$. (2011). A boundary condition capturing immersed interface method for 3D rigid objects in a flow. J. Comput. Phys. 230: 7176-7190.

Xu, S. and Pearson, G.D. (2015). Computing jump conditions for the immersed interface method using triangular meshes. J. Comput. Phys. 302: 59-67.

$\mathrm{Xu}, \mathrm{S}$. and Wang, Z.J. (2006). Systematic derivation of jump conditions for the immersed interface method in three-dimensional flow simulation. SIAM J. Sci. Comput. 27: 1948-1980.
Xu, S. and Wang, Z.J. (2008). A 3D immersed interface method for fluid-solid interaction. Comput. Meth. Appl. Mech. Eng. 197: 2068-2086.

Yang, G., Causon, D.M., and Ingram, D.M. (1999). Cartesian cut-cell method for axisymmetric separating body flows. AIAA J. 37: 905-911.

Yang, J. and Balaras, E. (2006). An embedded-boundary formulation for large-eddy simulation of turbulent flows interacting with moving boundaries. J. Comput. Phys. 215: 12-40.

Yang, J. and Stern, F. (2013). Fully resolved simulation OF particulate flow using a sharp interface direct forcing immersed boundary method. In: Presented at ASME 2013 Fluids Engineering Division Summer Meeting. American Society of Mechanical Engineers, Incline Village, Nevada, USA.

Yang, X., Zhang, X., Li, Z., and He, G.-W. (2009). A smoothing technique for discrete delta functions with application to immersed boundary method in moving boundary simulations. J. Comput. Phys. 228: 7821-7836.

Yao, L. and Fogelson, A.L. (2012). Simulations of chemical transport and reaction in a suspension of cells I: an augmented forcing point method for the stationary case. Int. J. Numer. Meth. Fluid. 69: 1736-1752.

Yao, L. and Mori, Y. (2017). A numerical method for osmotic water flow and solute diffusion with deformable membrane boundaries in two spatial dimension. J. Comput. Phys. 350: 728-746.

Ye, T., Mittal, R., Udaykumar, H.S., and Shyy, W. (1999). An accurate Cartesian grid method for viscous incompressible flows with complex immersed boundaries. J. Comput. Phys. 156: 209-240.

Yousefzadeh, M. and Battiato, I. (2019). High order ghost-cell immersed boundary method for generalized boundary conditions. Int. J. Heat Mass Tran. 137: 585-598.

Zaidi, A.A., Tsuji, T., and Tanaka, T. (2014). A new relation of drag force for high Stokes number monodisperse spheres by direct numerical simulation. Adv. Powder Technol. 25: 1860-1871.

Zhang, H., Luo, K., Haugen, N.E.L., Mao, C., and Fan, J. (2020). Drag force for a burning particle. Combust. Flame 217: 188-199.

Zeeuw, D.L. (1993). A quadtree-based adaptively refined Cartesiangrid algorithm for solution of the Euler equations. University of Michigan.

Zhang, L., Gerstenberger, A., Wang, X., and Liu, W.K. (2004). Immersed finite element method. Comput. Meth. Appl. Mech. Eng. 193: 2051-2067.

Zhang, L., Liu, K., and You, C. (2015). Fictitious domain method for fully resolved reacting gas-solid flow simulation. J. Comput. Phys. 299: 215-228.

Zhang, N. and Zheng, Z.C. (2007). An improved direct-forcing immersed-boundary method for finite difference applications. J. Comput. Phys. 221: 250-268.

Zhang, N., Zheng, Z.C., and Eckels, S. (2008). Study of heat-transfer on the surface of a circular cylinder in flow using an immersedboundary method. Int. J. Heat Fluid Flow 29: 1558-1566.

Zhong, X. (2007). A new high-order immersed interface method for solving elliptic equations with imbedded interface of discontinuity. J. Comput. Phys. 225: 1066-1099.

Zhu, L.-T., Liu, Y.-X., and Luo, Z.-H. (2019). An enhanced correlation for gas-particle heat and mass transfer in packed and fluidized bed reactors. Chem. Eng. J. 374: 531-544. 


\section{Bionotes}

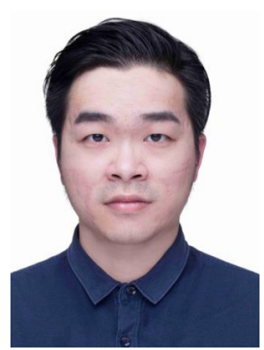

\section{Wei Xiao}

State Key Laboratory of Clean Energy Utilization, Zhejiang University, Hangzhou 310027, P.R. China

Wei Xiao received his B.E. degree from Zhejiang University in 2016. Since then, he has been a Ph.D. candidate in the State Key Laboratory of Clean Energy Utilization at Zhejiang University. His research interests include the immersed boundary method, boundary layer flow, and compressible multiphase turbulence.

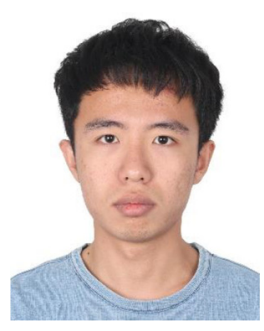

\section{Hancong Zhang}

State Key Laboratory of Clean Energy Utilization, Zhejiang University, Hangzhou 310027, P.R. China

Hancong Zhang is currently a Ph.D. candidate in the State Key Laboratory of Clean Energy Utilization at Zhejiang University. He is a thirdyear student. His research interests include the particle resolved simulation of reactive particles and the ghost-cell immersed boundary method

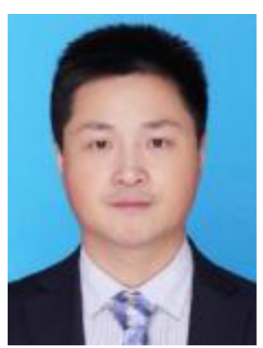

\section{Kun Luo}

State Key Laboratory of Clean Energy Utilization, Zhejiang University, Hangzhou 310027, P.R. China zjulk@zju.edu.cn https://orcid.org/0000-0002-2583-3776

Kun Luo is a full professor in the State Key Laboratory of Clean Energy Utilization at Zhejiang University, China, since 2010. He received his
B.S.E. from Wuhan University in 2000 and his Ph.D. from Zhejiang University in 2005. From 2007 to 2009, he worked at the Center for Turbulence Research, Stanford University as a postdoctoral fellow. His research centers on computational energy sciences with high-fidelity simulations on complex multiphase flows and combustion in energy and power system. He has won the Distinguished Paper Award from the 33rd International Symposium on Combustion and the Wu Zhonghua Excellent Young Scholar Award from Chinese Association of Engineering Thermal Physics in 2011.

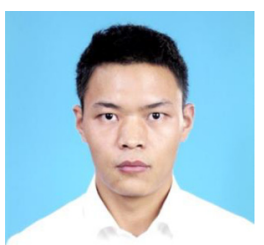

\section{Chaoli Mao}

State Key Laboratory of Clean Energy Utilization, Zhejiang University, Hangzhou 310027, P.R. China

Chaoli Mao received his B.E. degree from the College of Energy Engineering at Zhejiang University in 2014 and also his Ph.D. degree in 2019. His doctoral research focused on immersed boundary method, fully resolved simulation of reactive particle, and shock-particle interaction. He is now working as an algorithm engineer in CRRC.

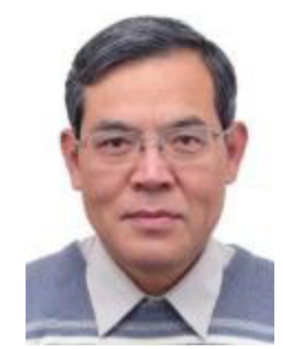

\section{Jianren Fan}

State Key Laboratory of Clean Energy Utilization, Zhejiang University, Hangzhou 310027, P.R. China

Jianren Fan is a Cheung Kong Scholar Professor in the Department of Energy, Zhejiang University. He received his B.S. degree from Xi'an Jiaotong University of China in 1981 and Ph.D. degree from the Department of Mechanical Engineering, Technical University of Vienna, Austria, in 1984. His current research interests are in numerical simulation and experimental studies on gas-solid twophase flows and combustion, including spray atomization, multiphase turbulence, heat/mass transfer, and chemical reaction in turbulent flows. He was awarded the Second-Class Prize of the National Natural Science of China in 2005. 